\title{
Efficient Synthesis of Chiral $\beta$-Hydroxy Sulfones via Ru-Catalyzed Highly Enantioselective Hydrogenation in the Presence of Iodine
}

Xiaobing Wan, ${ }^{\dagger}$ Qinghua Meng, ${ }^{\star}$ Hongwei Zhang, ${ }^{\star}$ Yanhui Sun,${ }^{\dagger}$ Weizheng Fan, ${ }^{\star}$ and Zhaoguo Zhang ${ }^{\dagger+*}$

School of Chemistry and Chemical Engineering, Shanghai Jiaotong University, 800 Dongchuan Road, Shanghai 200240, China, and Shanghai Institute of Organic Chemistry, 354 Fenglin Road, Shanghai 200032, China

zhaoguo@sjtu.edu.cn

\section{Table of contents}

\begin{tabular}{l|l}
\hline Table & Page \\
\hline General & S2 \\
\hline Analytical and spectral data for compounds 1, 2, 3, 4, 5 & S3-S9 \\
\hline HPLC data & S10-S40 \\
\hline NMR Spectrum of compounds $\mathbf{1 , 2 , 3 , 4 , 5}$ & S41-S100 \\
\hline
\end{tabular}




\section{Experimental Section}

General: All reactions were carried out under inert atmosphere of dry argon or nitrogen. THF and toluene were freshly distilled from sodium/benzophenone ketyl; $\mathrm{CH}_{2} \mathrm{Cl}_{2}$ was distilled from $\mathrm{CaH}_{2}$ under argon atmosphere; EtOH for catalyst preparation or hydrogenation was distilled from magnesium under atmosphere. The preparation of samples and the setup of high-pressure reactor were either carried out in a glovebox or using standard Schlenk-type techniques. ${ }^{1}$ HNMR $(300 / 400 \mathrm{MHz})$, ${ }^{13} \mathrm{C}$ NMR $(75.4 / 100 \mathrm{MHz})$ were registered with $\mathrm{CDCl}_{3}$ or DMSO as solvent. Chemical shifts are reported in units (ppm) by assigning TMS resonance in the ${ }^{1} \mathrm{H}$ spectrum as $0.00 \mathrm{ppm}$ and $\mathrm{CDCl}_{3}$ resonance in the ${ }^{13} \mathrm{C}$ spectrum as $77.0 \mathrm{ppm}$. All coupling constants ( $J$ values) were reported in Hertz $(\mathrm{Hz})$. Column chromatography was performed on silica gel 300-400 mesh. HPLC were performed by the State-authorized Analytical Center in Shanghai Institute of Organic Chemistry, Chinese Academy of Science.

\section{Experiment: asymmetric hydrogenation of $\beta$-sufonyl ketones}

To a $20 \mathrm{~mL}$ Schlenk tube were added $\left[\mathrm{Ru}(\text { benzene }) \mathrm{Cl}_{2}\right]_{2}(10 \mathrm{mg}, 0.02 \mathrm{mmol})$ and (S)-L3 (30 mg, $0.045 \mathrm{mmol})$. The tube was purged with Argon three times before addition of freshly distilled and degassed EtOH/Benzene $(8 \mathrm{~mL} / 1 \mathrm{~mL})$. The resulting mixture was heated at $50{ }^{\circ} \mathrm{C}$ for $1 \mathrm{~h}$. The catalyst was dried under reduced pressure and was taken into a glove box in a dry nitrogen atmosphere and dissolved in degassed ethanol $(8 \mathrm{~mL})$ which was then put into 4 vials equally. To these vials $\beta$-sufonyl ketones $(1 \mathrm{mmol})$ was introduced, and then the vials were taken into an autoclave. The autoclave was purged three times with $\mathrm{H}_{2}$, and the pressure of $\mathrm{H}_{2}$ was set to 150 psi before it was placed in an oil bath at designed temperature for $20 \mathrm{~h}$. Cooled to ambient temperature and the hydrogen gas was released carefully. The solvent was removed and the residue was passed through a silica gel column to give the product. Enantiomeric purity of the products was determined by HPLC. 
<smiles>O=C(CS(=O)(=O)c1ccccc1)c1ccccc1</smiles>

1-phenyl-2-(phenylsulfonyl)ethanone (1a)

${ }^{1} \mathrm{H}$ NMR (400 MHz, CDCl $)$ ): 4.74 (s, 2H), 7.45 7.67 (m, $\left.6 \mathrm{H}\right), 7.88 \sim 7.94$ (m, 4 H).

${ }^{13} \mathrm{C}$ NMR (100 MHz, $\left.\mathrm{CDCl}_{3}\right)$ : 63.1, 128.3, 128.7, 129.02, 129.05, 134.0, 134.2, 135.5, 138.6, 187.9.<smiles>O=S(=O)(CC(O)c1ccccc1)c1ccccc1</smiles>

1-phenyl-2-(phenylsulfonyl)ethanol (2a)

${ }^{1} \mathrm{H}$ NMR (400 MHz, $\mathrm{CDCl}_{3}$ ): 3.34 (dd, $\left.J=14.4 \mathrm{~Hz}, 1.6 \mathrm{~Hz}, 1 \mathrm{H}\right), 3.49$ (dd, $J=14.4 \mathrm{~Hz}, 10.4 \mathrm{~Hz}$, $1 \mathrm{H}), 3.67$ (brs, $1 \mathrm{H}), 5.27$ - 5.29 (m, $1 \mathrm{H}), 7.26$ - 7.34 (m, 5 H), 7.57 - 7.70 (m, 3 H), 7.95 - 7.99 (m, $2 \mathrm{H})$.

${ }^{13} \mathrm{C} \mathrm{NMR}\left(100 \mathrm{MHz}, \mathrm{CDCl}_{3}\right): 63.7,68.3,125.5,127.8,128.2,128.6,129.3,134.0,138.9,140.6$. HPLC (Chiralpak AD - H, elution with i-PrOH/hexane $=30 / 70$, flow rate: $0.6 \mathrm{~mL} / \mathrm{min}, \lambda=254 \mathrm{~nm}$ ): $\mathrm{t}_{1}: 19.0 \mathrm{~min}, \mathrm{t}_{2}: 21.7 \mathrm{~min}$.<smiles>O=C(CS(=O)(=O)c1ccccc1)c1ccc(F)cc1</smiles>

1-(4-fluorophenyl)-2-(phenylsulfonyl)ethanone (1b)

${ }^{1} \mathrm{H}$ NMR (400 MHz, $\mathrm{CDCl}_{3}$ ): 4.71 (s, $\left.2 \mathrm{H}\right), 7.13-7.17$ (m, $\left.2 \mathrm{H}\right), 7.53-8.00$ (m, 7H).

${ }^{13} \mathrm{C}$ NMR (100 MHz, $\left.\mathrm{CDCl}_{3}\right): 63.1,115.8,128.2,129.0,131.9,132.0,134.1,138.5,164.9,167.4$, 186.4 .<smiles>O=S(=O)(CC(O)c1ccc(F)cc1)c1ccccc1</smiles>

1-(4-fluorophenyl)-2-(phenylsulfonyl)ethanol (2b)

${ }^{1} \mathrm{H}$ NMR (400 MHz, $\mathrm{CDCl}_{3}$ ): 3.31 (dd, $J=14.4 \mathrm{~Hz}, 1.6 \mathrm{~Hz}, 1 \mathrm{H}$ ), 3.47 (dd, $J=14.4 \mathrm{~Hz}, 10.0 \mathrm{~Hz}$, $1 \mathrm{H}), 3.73(\mathrm{~d}, J=2.0 \mathrm{~Hz}, 1 \mathrm{H}), 5.27-5.29(\mathrm{~m}, 1 \mathrm{H}), 6.99-7.97(\mathrm{~m}, 9 \mathrm{H})$.

${ }^{13} \mathrm{C}$ NMR (100 MHz, $\left.\mathrm{CDCl}_{3}\right)$ : 63.7, 67.7, 115.5, 127.4, 127.9, 129.4, 134.1, 136.5, 139.0, 163.6, 161.2 .

HPLC (Chiralpak AD - H, elution with i-PrOH $/$ hexane $=30 / 70$, flow rate: $0.6 \mathrm{~mL} / \mathrm{min}, \lambda=254 \mathrm{~nm}$ ): $\mathrm{t}_{1}: 21.5 \mathrm{~min}, \mathrm{t}_{2}: 24.2 \mathrm{~min}$.<smiles>O=C(CS(=O)(=O)c1ccccc1)c1ccc(Cl)cc1</smiles>

1-(4-chlorophenyl)-2-(phenylsulfonyl)ethanone (1c)

${ }^{1} \mathrm{H}$ NMR (400 MHz, $\left.\mathrm{CDCl}_{3}\right)$ : 4.71 (s, $\left.2 \mathrm{H}\right), 7.44$ - 7.71 (m, $\left.5 \mathrm{H}\right), 7.86$ - 7.91 (m, $\left.4 \mathrm{H}\right)$. 
${ }^{13} \mathrm{C}$ NMR (100 MHz, $\left.\mathrm{CDCl}_{3}\right)$ : 63.2, 128.3, 129.0, 129.1, 130.5, 133.8, 134.1, 138.4, 140.8, 186.8.<smiles>O=S(=O)(CC(O)c1ccc(Cl)cc1)c1ccccc1</smiles>

1-(4-chlorophenyl)-2-(phenylsulfonyl)ethanol (2c)

${ }^{1} \mathrm{H}$ NMR (400 MHz, $\mathrm{CDCl}_{3}$ ): 3.31 (dd, $\left.J=14.4 \mathrm{~Hz}, 2.0 \mathrm{~Hz}, 1 \mathrm{H}\right), 3.46$ (dd, $J=14.4 \mathrm{~Hz}, 10.4 \mathrm{~Hz}$, $1 \mathrm{H}), 3.75(\mathrm{~d}, J=2.0 \mathrm{~Hz}, 1 \mathrm{H}), 5.26-5.29(\mathrm{~m}, 1 \mathrm{H}), 7.23-7.97(\mathrm{~m}, 9 \mathrm{H})$.

${ }^{13} \mathrm{C}$ NMR (100 MHz, $\left.\mathrm{CDCl}_{3}\right): 63.6,67.7,127.0,127.9,128.8,129.4,133.9,134.1,138.9,139.1$.

HPLC (Chiralcel OJ - H, elution with i-PrOH/hexane $=40 / 60$, flow rate: $0.8 \mathrm{~mL} / \mathrm{min}, \lambda=254 \mathrm{~nm}$ ): $\mathrm{t}_{1}: 17.2 \mathrm{~min}, \mathrm{t}_{2}: 20.1 \mathrm{~min}$.<smiles>O=C(CS(=O)(=O)c1ccccc1)c1ccc(Br)cc1</smiles>

1-(4-bromophenyl)-2-(phenylsulfonyl)ethanone (1d)

${ }^{1} \mathrm{H}$ NMR (400 MHz, $\left.\mathrm{CDCl}_{3}\right): 4.70$ (s, $\left.2 \mathrm{H}\right), 7.54-7.88$ (m, $\left.9 \mathrm{H}\right)$

${ }^{13} \mathrm{C}$ NMR $\left(100 \mathrm{MHz}, \mathrm{CDCl}_{3}\right): 63.3,128.4,129.2,129.8,130.6,130.6,132.1,134.2,134.3,138.4$, 187.0<smiles>O=S(=O)(CC(O)c1ccc(Br)cc1)c1ccccc1</smiles>

1-(4-bromophenyl)-2-(phenylsulfonyl)ethanol (2d)

${ }^{1} \mathrm{H}$ NMR (400 MHz, $\mathrm{CDCl}_{3}$ ): 3.31 (dd, $J=14.4 \mathrm{~Hz}, 1.6 \mathrm{~Hz}, 1 \mathrm{H}$ ), 3.45 (dd, $J=14.4 \mathrm{~Hz}, 10.0 \mathrm{~Hz}$, $1 \mathrm{H}), 3.75(\mathrm{~d}, J=2.0 \mathrm{~Hz}, 1 \mathrm{H}), 5.25-5.27(\mathrm{~m}, 1 \mathrm{H}), 7.17-7.96(\mathrm{~m}, 9 \mathrm{H})$.

${ }^{13} \mathrm{C}$ NMR (100 MHz, $\left.\mathrm{CDCl}_{3}\right)$ : 63.5, 67.7, 122.0, 127.3, 127.8, 129.4, 131.7, 134.1, 138.9, 139.7.

HPLC (Chiralcel OJ-H, elution with i-PrOH/hexane $=40 / 60$, flow rate: $0.6 \mathrm{~mL} / \mathrm{min}, \lambda=254 \mathrm{~nm}$ ): $\mathrm{t}_{1}$ : $25.5 \mathrm{~min}, \mathrm{t}_{2}: 30.9 \mathrm{~min}$.

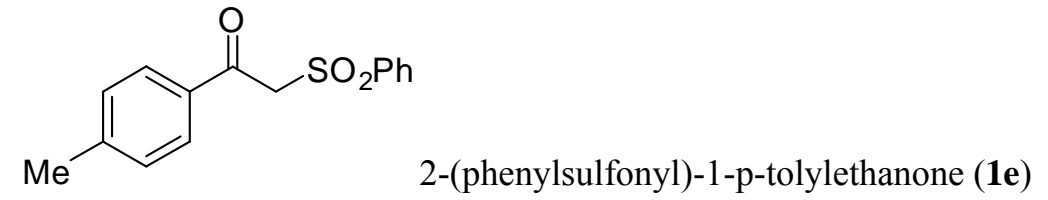

${ }^{1} \mathrm{H}$ NMR (400 MHz, $\mathrm{CDCl}_{3}$ ): 2.41 (s, $\left.3 \mathrm{H}\right), 4.72$ (s, $\left.2 \mathrm{H}\right), 7.25$ - 7.90 (m, $9 \mathrm{H}$ ).

${ }^{13} \mathrm{C}$ NMR (100 MHz, $\left.\mathrm{CDCl}_{3}\right): 21.5,62.9,128.2,128.9,129.1,129.3,133.0,133.9,138.6,145.3$, 187.3 .<smiles>Cc1ccc(C(O)CS(=O)(=O)c2ccccc2)cc1</smiles>

${ }^{1} \mathrm{H}$ NMR (400 MHz, CDCl 3 ): 2.31 (s, $\left.3 \mathrm{H}\right), 3.33$ (dd, $\left.J=14.4 \mathrm{~Hz}, 2.0 \mathrm{~Hz}, 1 \mathrm{H}\right), 3.50$ (dd, $J=14.4$ Hz, 10.4 Hz, 1 H), 3.58 (br, 1 H), 5.23 - 5.25 (m, 1 H), 7.12 - 7.97 (m, 9 H). 
${ }^{13} \mathrm{C}$ NMR (100 MHz, $\left.\mathrm{CDCl}_{3}\right): 21.0,63.8,68.2,125.5,127.9,129.3,129.4,134.0,137.7,138.1$, 139.1.

HPLC (Chiralpak AD-H, elution with i-PrOH/hexane $=40 / 60$, flow rate: $0.3 \mathrm{~mL} / \mathrm{min}, \lambda=254 \mathrm{~nm}$ ): $\mathrm{t}_{1}: 32.5 \mathrm{~min}, \mathrm{t}_{2}: 35.8 \mathrm{~min}$.<smiles>COc1ccc(C(=O)CS(=O)(=O)c2ccccc2)cc1</smiles>

1-(4-methoxyphenyl)-2-(phenylsulfonyl)ethanone (1f)

${ }^{1} \mathrm{H}$ NMR (400 MHz, $\left.\mathrm{CDCl}_{3}\right): 3.87$ (s, $\left.3 \mathrm{H}\right), 4.68$ (s, $\left.2 \mathrm{H}\right), 6.92$ - 6.95 (m, $\left.2 \mathrm{H}\right), 7.51$ - 7.67 (m, 3 H), 7.87 - 7.93 (m, $4 \mathrm{H})$.

${ }^{13} \mathrm{C}$ NMR (100 MHz, $\left.\mathrm{CDCl}_{3}\right): 55.5,63.1,113.9,128.3,128.7,129.0,131.7,134.0,138.6,164.4$, 186.0.

1-(4-methoxyphenyl)-2-(phenylsulfonyl)ethanol (2f)

${ }^{1} \mathrm{H}$ NMR (400 MHz, $\mathrm{CDCl}_{3}$ ): 3.32 (dd, $\left.J=14.4 \mathrm{~Hz}, 1.6 \mathrm{~Hz}, 1 \mathrm{H}\right), 3.51$ (dd, $J=14.4 \mathrm{~Hz}, 10.0 \mathrm{~Hz}$, $1 \mathrm{H}), 3.59-3.60$ (m, $1 \mathrm{H}), 3.77$ (s, $3 \mathrm{H}), 5.21$ - 5.24 (m, $1 \mathrm{H}), 7.17$ - $7.96(\mathrm{~m}, 9 \mathrm{H})$.

${ }^{13} \mathrm{C}$ NMR (100 MHz, $\left.\mathrm{CDCl}_{3}\right): 55.0,63.6,67.9,113.8,126.8,127.7,129.1,132.9,133.7,139.1$, 159.2 .

HPLC (Chiralcel OJ - H, elution with i-PrOH/hexane $=40 / 60$, flow rate: $0.6 \mathrm{~mL} / \mathrm{min}, \lambda=254 \mathrm{~nm}$ ): $\mathrm{t}_{1}: 27.1 \mathrm{~min}, \mathrm{t}_{2}: 35.6 \mathrm{~min}$.<smiles>O=C(CS(=O)(=O)c1ccccc1)c1cccc(Cl)c1</smiles>

1-(3-chlorophenyl)-2-(phenylsulfonyl)ethanone (1g)

${ }^{1} \mathrm{H}$ NMR (400 MHz, $\left.\mathrm{CDCl}_{3}\right): 4.71$ (s, $\left.2 \mathrm{H}\right), 7.42$ - 7.90 (m, $\left.9 \mathrm{H}\right)$.

${ }^{13} \mathrm{C}$ NMR (100 MHz, $\mathrm{CDCl}_{3}$ ): 63.4, 127.5, 128.46, 128.48, 129.0, 129.2, 130.1, 134.2, 134.4, $135.2,137.1,138.4,186.9$.<smiles>O=S(=O)(CC(O)c1cccc(Cl)c1)c1ccccc1</smiles>

1-(3-chlorophenyl)-2-(phenylsulfonyl)ethanol (2g)

${ }^{1} \mathrm{H}$ NMR (400 MHz, $\left.\mathrm{CDCl}_{3}\right): 3.32(\mathrm{dd}, J=14.0 \mathrm{~Hz}, 1.6 \mathrm{~Hz}, 1 \mathrm{H}), 3.46(\mathrm{dd}, J=14.0 \mathrm{~Hz}, 10.0 \mathrm{~Hz}$, $1 \mathrm{H}), 3.77(\mathrm{~d}, J=2.0 \mathrm{~Hz}, 1 \mathrm{H}), 5.26-5.28(\mathrm{~m}, 1 \mathrm{H}), 7.16-7.97(\mathrm{~m}, 9 \mathrm{H})$.

${ }^{13} \mathrm{C}$ NMR $\left(100 \mathrm{MHz}, \mathrm{CDCl}_{3}\right): 63.6,67.8,123.8,125.9,127.9,128.3,129.4,130.0,134.2,134.6$, 138.9, 142.6 .

HPLC (Chiralpak AD - H, elution with i-PrOH/hexane $=30 / 70$, flow rate: $0.6 \mathrm{~mL} / \mathrm{min}, \lambda=254 \mathrm{~nm}$ ): $\mathrm{t}_{1}: 15.1 \mathrm{~min}, \mathrm{t}_{2}: 18.2 \mathrm{~min}$. 
<smiles>Cc1ccccc1C(=O)CS(=O)(=O)c1ccccc1</smiles>

2-(phenylsulfonyl)-1-o-tolylethanone (1h)

${ }^{1} \mathrm{H}$ NMR (400 MHz, CDCl $)$ ): 2.43 (s, $\left.3 \mathrm{H}\right), 4.71$ (s, $\left.2 \mathrm{H}\right), 7.24$ - 7.89 (m, $9 \mathrm{H}$ ).

${ }^{13} \mathrm{C}$ NMR $\left(100 \mathrm{MHz}, \mathrm{CDCl}_{3}\right)$ : 21.5, 65.4, 125.9, 128.4, 129.2, 130.3, 132.3, 132.8, 134.1, 135.6, $138.9,140.0,190.3$<smiles>Cc1ccccc1C(O)CS(=O)(=O)c1ccccc1</smiles>

2-(phenylsulfonyl)-1-o-tolylethanol (2h)

${ }^{1} \mathrm{H}$ NMR (400 MHz, $\mathrm{CDCl}_{3}$ ): 2.08 (s, $\left.3 \mathrm{H}\right), 3.26$ (dd, $\left.J=14.4 \mathrm{~Hz}, 1.6 \mathrm{~Hz}, 1 \mathrm{H}\right), 3.43$ (dd, $J=14.4$ $\mathrm{Hz}, 10.0 \mathrm{~Hz}, 1 \mathrm{H}), 3.63$ (d, $J=2.4 \mathrm{~Hz}, 1 \mathrm{H}), 5.43-5.46$ (m, $1 \mathrm{H}), 7.07-8.00$ (m, $9 \mathrm{H})$.

${ }^{13} \mathrm{C}$ NMR (100 MHz, $\left.\mathrm{CDCl}_{3}\right): 18.4,62.7,64.9,125.2,126.5,127.9,129.4,130.4,133.6,134.0$, 138.6, 138.8 .

HPLC (Chiralpak AD - H, elution with i-PrOH/hexane $=40 / 60$, flow rate: $0.6 \mathrm{~mL} / \mathrm{min}, \lambda=254 \mathrm{~nm}$ ): $\mathrm{t}_{1}: 14.7 \mathrm{~min}, \mathrm{t}_{2}: 16.9 \mathrm{~min}$.<smiles>O=C(CS(=O)(=O)c1ccccc1)c1ccccc1Cl</smiles>

1-(2-chlorophenyl)-2-(phenylsulfonyl)ethanone (1i)

${ }^{1} \mathrm{H}$ NMR (400 MHz, $\left.\mathrm{CDCl}_{3}\right)$ : 4.84(s, $\left.2 \mathrm{H}\right), 7.32-7.90$ (m, $\left.9 \mathrm{H}\right)$

${ }^{13} \mathrm{C}$ NMR $\left(100 \mathrm{MHz}, \mathrm{CDCl}_{3}\right)$ : 66.2, 127.1, 128.4, 129.1, 130.4, 130.6, 131.4, 133.1, 134.1, 137.1, 138.8, 190.0.<smiles>O=S(=O)(CC(O)c1ccccc1Cl)c1ccccc1</smiles>

1-(2-chlorophenyl)-2-(phenylsulfonyl)ethanol (2i)

${ }^{1} \mathrm{H}$ NMR (400 MHz, $\mathrm{CDCl}_{3}$ ): 3.30 (dd, $\left.J=14.4 \mathrm{~Hz}, 10.0 \mathrm{~Hz}, 1 \mathrm{H}\right), 3.52(\mathrm{dd}, J=14.4 \mathrm{~Hz}, 1.2 \mathrm{~Hz}$, $1 \mathrm{H}), 3.97(\mathrm{~d}, \mathrm{~J}=2.4 \mathrm{~Hz}, 1 \mathrm{H}), 5.42-5.44(\mathrm{~m}, 1 \mathrm{H}), 7.19-8.02(\mathrm{~m}, 9 \mathrm{H})$.

${ }^{13} \mathrm{C}$ NMR $\left(100 \mathrm{MHz}, \mathrm{CDCl}_{3}\right)$ : 61.7, 65.3, 127.1, 127.3, 128.1, 129.2, 129.3, 129.4, 130.7, 134.1, 137.7, 138.4 .

HPLC (Chiralpak AD - H, elution with i-PrOH/hexane $=40 / 60$, flow rate: $0.6 \mathrm{~mL} / \mathrm{min}, \lambda=254 \mathrm{~nm}$ ): $\mathrm{t}_{1}: 14.5 \mathrm{~min}, \mathrm{t}_{2}: 19.7 \mathrm{~min}$.

少 $\mathrm{SO}_{2} \mathrm{Ph}_{1-(\text { phenylsulfonyl)propan-2-one (1j) }}$

${ }^{1} \mathrm{H}$ NMR (400 MHz, $\mathrm{CDCl}_{3}$ ): 2.39 (s, 3H), 4.16 (s, 2H), 7.56 7.90 (m, $5 \mathrm{H}$ ).

${ }^{13} \mathrm{C}$ NMR (100 MHz, $\left.\mathrm{CDCl}_{3}\right): 31.3,67.3,129.2,134.1,138.4,195.9$. 


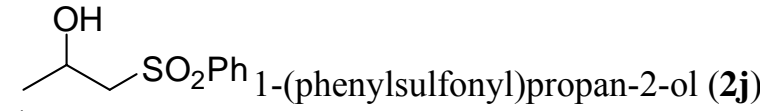

${ }^{1} \mathrm{H}$ NMR (400 MHz, $\left.\mathrm{CDCl}_{3}\right): 1.24(\mathrm{~d}, 2 \mathrm{H}, J=6.4 \mathrm{~Hz}), 3.14 \sim 3.27(\mathrm{~m}, 2 \mathrm{H}), 3.44(\mathrm{br}, 1 \mathrm{H})$, 4.29 4.36 (m, 1H), 7.57 7.95 (m, 5H).

${ }^{13} \mathrm{C}$ NMR (100 MHz, $\left.\mathrm{CDCl}_{3}\right): 62.2,63.1,127.7,129.3,134.0,138.9$.

HPLC (Chiralpak AD - H, elution with i-PrOH/hexane $=30 / 70$, flow rate: $0.6 \mathrm{~mL} / \mathrm{min}, \lambda=254 \mathrm{~nm}$ ): $\mathrm{t}_{1}: 15.7 \mathrm{~min}, \mathrm{t}_{2}: 26.3 \mathrm{~min}$.<smiles>COS(=O)(=O)CC(=O)C(C)C</smiles>

3-methyl-1-(phenylsulfonyl)butan-2-one (1k)

${ }^{1} \mathrm{H}$ NMR $\left(400 \mathrm{MHz}, \mathrm{CDCl}_{3}\right): 1.10(\mathrm{~d}, J=6.4 \mathrm{~Hz}, 6 \mathrm{H}), 2.87 \sim 2.94(\mathrm{~m}, 1 \mathrm{H}), 4.23(\mathrm{~s}, 2 \mathrm{H})$, 7.55 7.91 (m, $5 \mathrm{H})$.

${ }^{13} \mathrm{C}$ NMR (100 MHz, $\left.\mathrm{CDCl}_{3}\right): 17.4,41.8,64.4,128.2,129.2,134.1,138.8,201.9$.<smiles>CC(C)C(O)CS(=O)(=O)c1ccccc1</smiles>

3-methyl-1-(phenylsulfonyl)butan-2-ol (2k)

${ }^{1} \mathrm{H}$ NMR $\left(400 \mathrm{MHz}, \mathrm{CDCl}_{3}\right): 0.87(\mathrm{~d}, J=7.2 \mathrm{~Hz}, 2 \mathrm{H}), 0.89(\mathrm{~d}, J=7.2 \mathrm{~Hz}, 2 \mathrm{H}), 1.70 \sim 1.78(\mathrm{~m}, 1 \mathrm{H})$, $3.18 \sim 3.25(\mathrm{~m}, 3 \mathrm{H}), 3.94 \sim 3.98(\mathrm{~m}, 1 \mathrm{H}), 7.58 \sim 7.95(\mathrm{~m}, 5 \mathrm{H})$,

${ }^{13} \mathrm{C}$ NMR $\left(100 \mathrm{MHz}, \mathrm{CDCl}_{3}\right): 16.7,17.7,33.1,59.8,69.9,127.6,129.1,133.7,139.0$.

HPLC (Chiralpak AD - H, elution with i-PrOH/hexane $=30 / 70$, flow rate: $0.6 \mathrm{~mL} / \mathrm{min}, \lambda=254 \mathrm{~nm}$ ): $\mathrm{t}_{1}: 19.3 \mathrm{~min}, \mathrm{t}_{2}: 26.9 \mathrm{~min}$.<smiles>O=C(CS(=O)(=O)c1ccccc1)C1CCCCC1</smiles>

1-cyclohexyl-2-(phenylsulfonyl)ethanone (11)

${ }^{1} \mathrm{H}$ NMR (400 MHz, $\mathrm{CDCl}_{3}$ ): 1.13 1.29 (m, $\left.5 \mathrm{H}\right), 1.61 \sim 1.87$ (m, $\left.5 \mathrm{H}\right), 2.61 \sim 2.66$ (m, $\left.1 \mathrm{H}\right), 4.21$ (s, 2H), 7.55 7.90 (m, $5 \mathrm{H})$.

${ }^{13} \mathrm{C}$ NMR (100 MHz, $\left.\mathrm{CDCl}_{3}\right): 25.1,25.5,27.7,51.3,64.5,128.2,129.1,134.1,138.8,201.1$.<smiles>O=S(=O)(CC(O)C1CCCCC1)c1ccccc1</smiles>

1-cyclohexyl-2-(phenylsulfonyl)ethanol (2l)

${ }^{1} \mathrm{H}$ NMR (400 MHz, $\left.\mathrm{CDCl}_{3}\right)$ : 0.94 1.73 (m, 11H), 3.21 3.24 (m, 3H), 3.93 3.95 (m, 1H), 7.57 7.94 (m, 5H).

${ }^{13} \mathrm{C}$ NMR (100 MHz, $\left.\mathrm{CDCl}_{3}\right)$ : 25.5, 25.6, 25.9, 27.1, 28.1, 42.8, 59.9, 69.4, 127.6, 129.1, 133.6, 139.0.

HPLC (Chiralpak AD - H, Chiralpak AD - H, elution with i-PrOH/hexane $=30 / 70$, flow rate: 0.6 $\mathrm{mL} / \mathrm{min}, \lambda=254 \mathrm{~nm}): \mathrm{t}_{1}: 15.5 \mathrm{~min}, \mathrm{t}_{2}: 18.2 \mathrm{~min}$. 
<smiles>O=C(c1ccccc1)C(I)S(=O)(=O)c1ccccc1</smiles>

2-iodo-1-phenyl-2-(phenylsulfonyl)ethanone (4a)

${ }^{1} \mathrm{H}$ NMR (300 MHz, DMSO): 6.83 (s, $\left.1 \mathrm{H}\right), 7.40-7.68$ (m, $\left.6 \mathrm{H}\right)$, 7.87-7.96. (m, $\left.4 \mathrm{H}\right)$.

${ }^{13} \mathrm{C}$ NMR (7 MHz, DMSO): 128.1, 128.9, 129.1, 129.2, 129.7, 133.32, 133.34, 134.6, 136.0, 189.5.<smiles>O=C(c1ccccc1)C(Br)S(=O)(=O)c1ccccc1</smiles>

2-bromo-1-phenyl-2-(phenylsulfonyl)ethanone (4b)

${ }^{1} \mathrm{H}$ NMR (400 MHz, $\left.\mathrm{CDCl}_{3}\right): 6.25(\mathrm{~s}, 1 \mathrm{H}), 7.49 \sim 8.00(\mathrm{~m}, 10 \mathrm{H})$.

${ }^{13} \mathrm{C}$ NMR $\left(100 \mathrm{MHz}, \mathrm{CDCl}_{3}\right)$ : 59.7, 128.8, 128.9, 129.3, 130.6, 134.0, 134.7, 134.8, 134.9, 186.4 .<smiles>CC(C)(C(=O)c1ccccc1)S(=O)(=O)c1ccccc1</smiles>

2-methyl-1-phenyl-2-(phenylsulfonyl)propan-1-one (4c)

${ }^{1} \mathrm{H}$ NMR (400 MHz, $\left.\mathrm{CDCl}_{3}\right): 1.71(\mathrm{~s}, 6 \mathrm{H}), 7.43 \sim 7.95$ (m, $\left.10 \mathrm{H}\right)$.

${ }^{13} \mathrm{C}$ NMR (100 MHz, $\left.\mathrm{CDCl}_{3}\right)$ : 22.4, 73.1, 128.0, 128.6, 130.3, 131.9, 134.0, 135.0, 137.5, 199.0.<smiles>CC(C)(C(O)c1ccccc1)S(=O)(=O)c1ccccc1</smiles>

2-methyl-1-phenyl-2-(phenylsulfonyl)propan-1-ol (5c)

${ }^{1} \mathrm{H}$ NMR (400 MHz, $\mathrm{CDCl}_{3}$ ): 0.94 (s, 3H), 1.41 (s, 3H), 4.27 (s, 1H), 5.19 (s, 1H), 7.28 7.97 (m, $10 \mathrm{H})$.

${ }^{13} \mathrm{C}$ NMR $\left(100 \mathrm{MHz}, \mathrm{CDCl}_{3}\right): 13.8,21.5,66.6,74.6,127.88,127.92,128.2,129.0,130.4,134.1$, 134.8, 137.8 .

HPLC (Chiralpak AD - H, Chiralpak AD - H, elution with i-PrOH/hexane $=30 / 70$, flow rate: 0.6 $\mathrm{mL} / \mathrm{min}, \lambda=254 \mathrm{~nm}): \mathrm{t}_{1}: 12.6 \mathrm{~min}, \mathrm{t}_{2}: 17.1 \mathrm{~min}$.<smiles>O=C(c1ccccc1)C(Cl)S(=O)(=O)c1ccccc1</smiles>

2-chloro-1-phenyl-2-(phenylsulfonyl)ethanone (4d)

${ }^{1} \mathrm{H}$ NMR (400 MHz, $\left.\mathrm{CDCl}_{3}\right): 6.22(\mathrm{~s}, 1 \mathrm{H}), 7.52 \sim 8.05(\mathrm{~m}, 10 \mathrm{H})$.

${ }^{13} \mathrm{C}$ NMR $\left(100 \mathrm{MHz}, \mathrm{CDCl}_{3}\right)$ : 71.7, 128.87, 128.90, 129.5, 130.5, 134.4, 134.8, 135.1, 186.1.<smiles>O=S(=O)(Oc1ccccc1)C(Cl)C(O)c1ccccc1</smiles>

2-chloro-1-phenyl-2-(phenylsulfonyl)ethanol (5d) 
${ }^{1} \mathrm{H}$ NMR $\left(400 \mathrm{MHz}, \mathrm{CDCl}_{3}\right): 3.23(\mathrm{~d}, J=3.2 \mathrm{~Hz}, 1 \mathrm{H}), 4.76(\mathrm{~d}, J=1.2 \mathrm{~Hz}, 1 \mathrm{H}), 5.92(\mathrm{~d}, J=3.2$ $\mathrm{Hz}, 1 \mathrm{H}), 7.32-8.04(\mathrm{~m}, 10 \mathrm{H})$;

$4.25(\mathrm{~d}, J=2.0 \mathrm{~Hz}, 1 \mathrm{H}), 4.82(\mathrm{~d}, J=9.2 \mathrm{~Hz}, 1 \mathrm{H}), 5.18(\mathrm{dd}, J=2.0 \mathrm{~Hz}, 9.2 \mathrm{~Hz}, 1 \mathrm{H}), 7.32-8.04(\mathrm{~m}$, $10 \mathrm{H})$.

${ }^{13} \mathrm{C}$ NMR (100 MHz, $\left.\mathrm{CDCl}_{3}\right): 69.4,73.8,75.6,78.6,126.0,127.5,128.3,128.4,128.9,129.0$, 129.1, 129.7, 134.7, 135.2, 137.9, 138.2.

HPLC (Chiralpak AD - H, Chiralpak AD - H, elution with i-PrOH/hexane $=30 / 70$, flow rate: 0.6 $\mathrm{mL} / \mathrm{min}, \lambda=254 \mathrm{~nm}): \mathrm{t}_{1}: 13.8 \mathrm{~min}, \mathrm{t}_{2}: 15.2 \mathrm{~min}, \mathrm{t}_{3}: 16.2 \mathrm{~min}, \mathrm{t}_{4}: 21.3 \mathrm{~min}$.<smiles>CC(C(=O)c1ccccc1)S(=O)(=O)c1ccccc1</smiles>

1-phenyl-2-(phenylsulfonyl)propan-1-one (4e)

${ }^{1} \mathrm{H}$ NMR (400 MHz, $\left.\mathrm{CDCl}_{3}\right): 1.57$ (d, $\left.J=7.2 \mathrm{~Hz}, 2 \mathrm{H}\right), 5.17$ (q, $\left.J=7.2 \mathrm{~Hz}, 1 \mathrm{H}\right), 7.46 \sim 7.67$ (m, 6 $\mathrm{H}), 7.78 \sim 7.98$ (m, $4 \mathrm{H})$.

${ }^{13} \mathrm{C}$ NMR $\left(100 \mathrm{MHz}, \mathrm{CDCl}_{3}\right): 12.8,64.6,128.5,128.7,128.8,129.4,133.8,134.0,135.9,136.0$, 192.3 . 


\section{HPLC data of Table1}<smiles>O=S(=O)(CC(O)c1ccccc1)c1ccccc1</smiles>

\section{2a racemate}

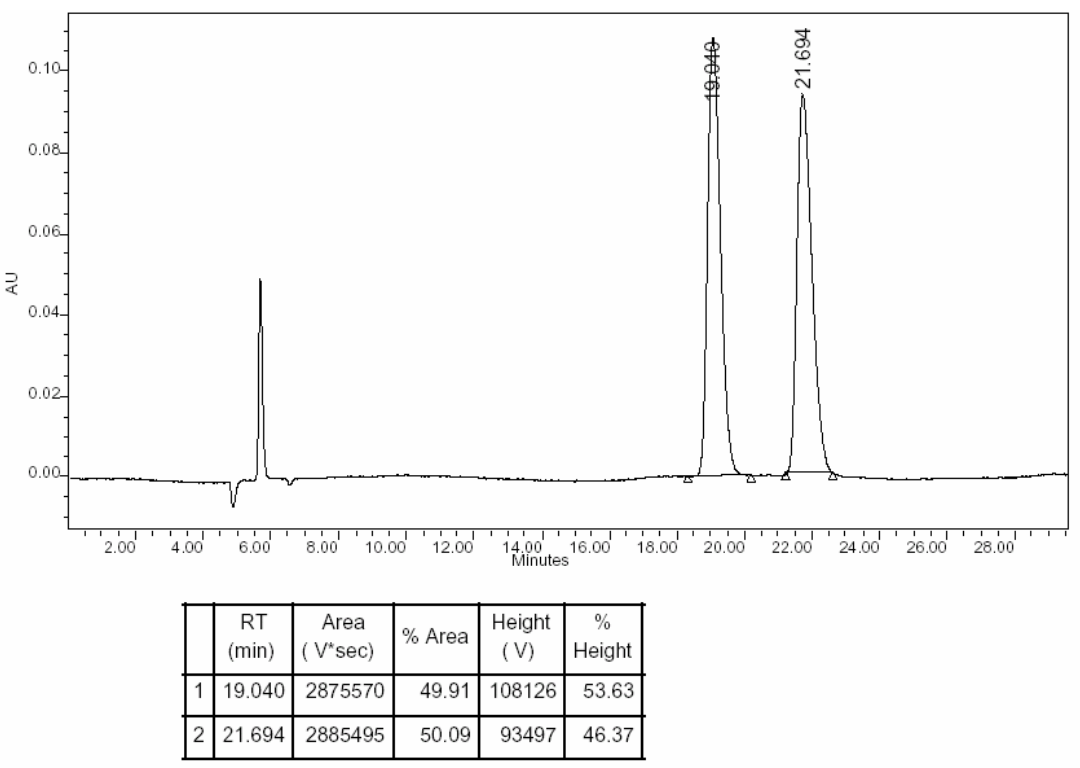

Table 1 entry 1

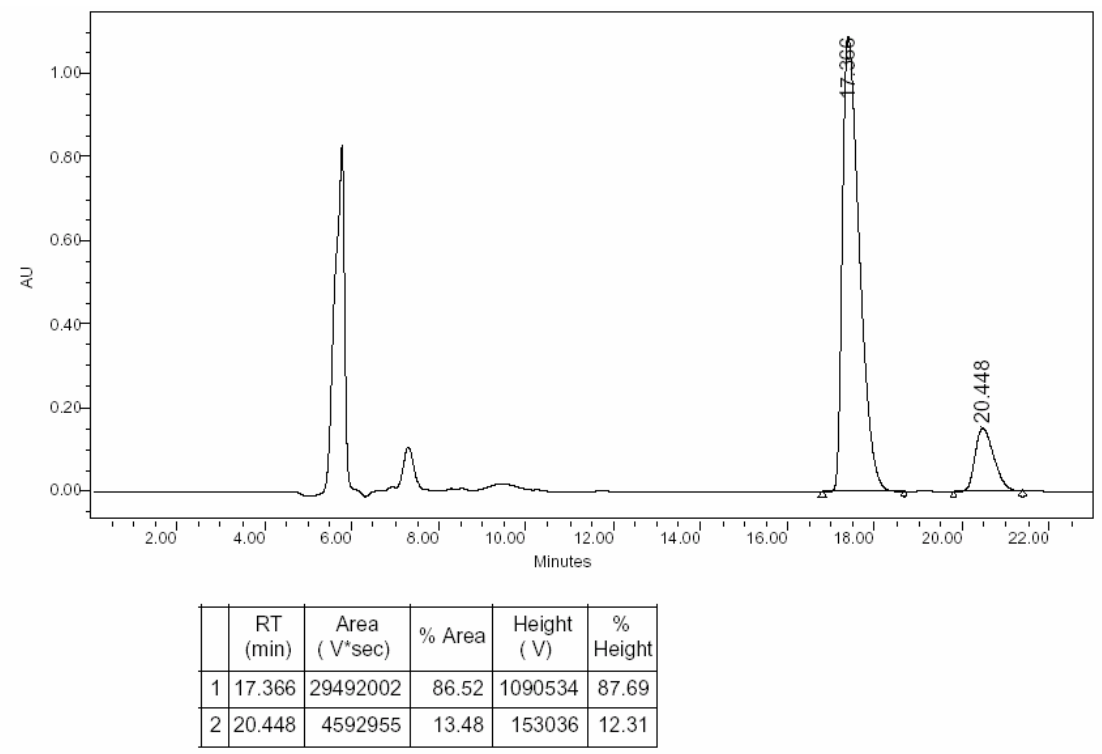


Table 1 entry 2

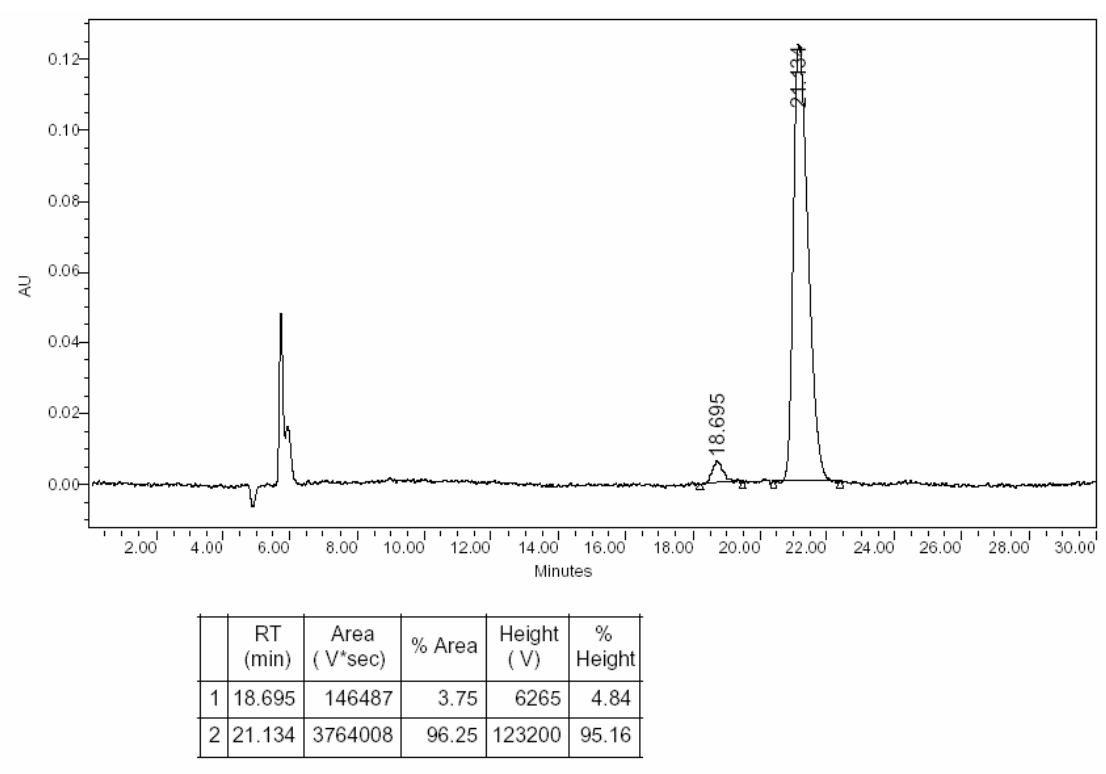

Table 1 entry 3

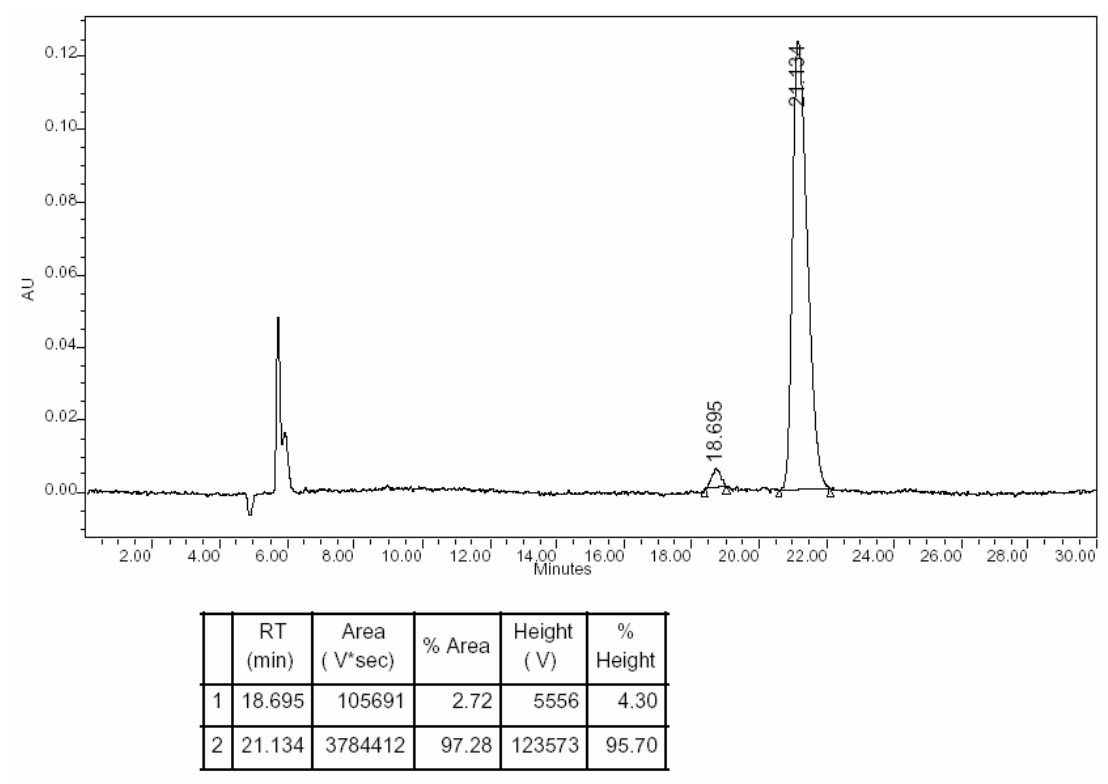


Table 1 entry 4

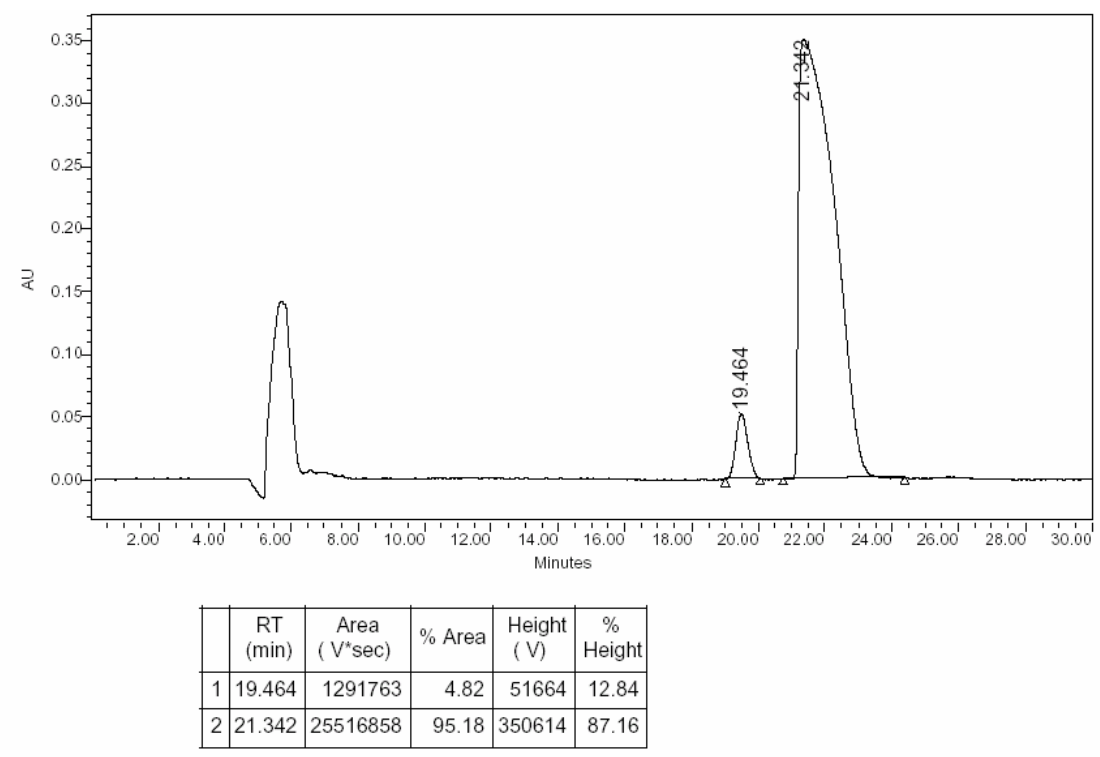

Table 1 entry 5

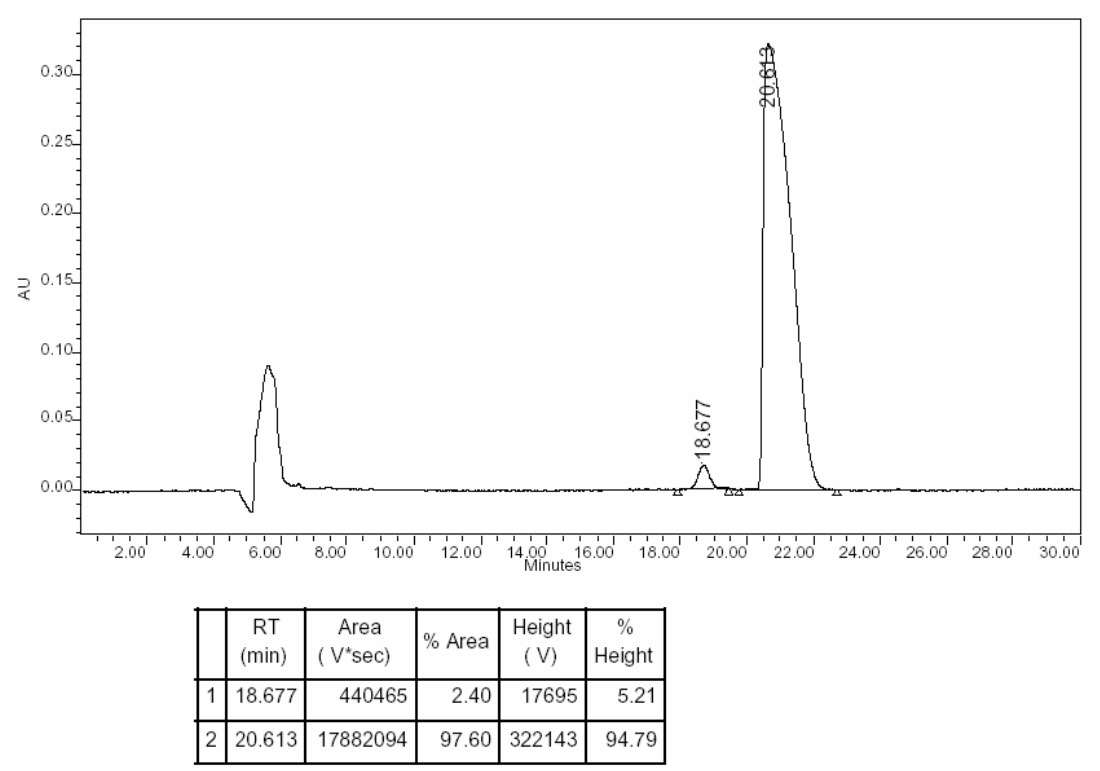


Table 1 entry 6

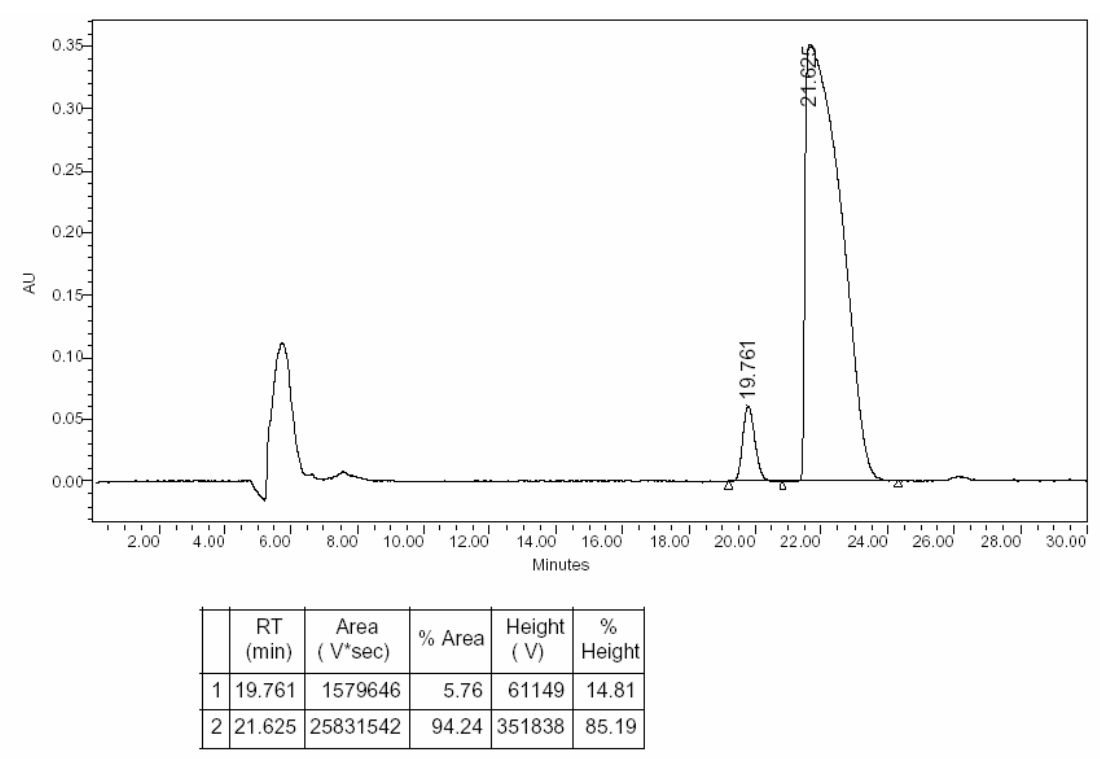

\section{HPLC and NMR data of Table2}

Table 2 entry 1

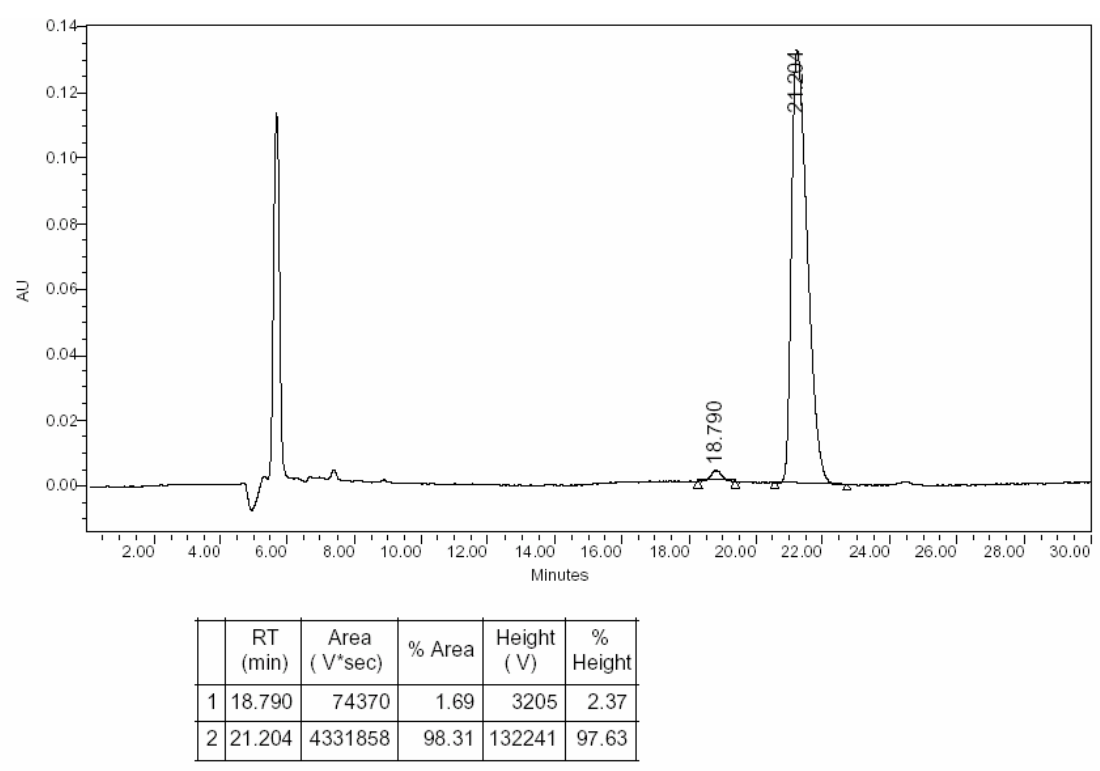


Table 2 entry 2

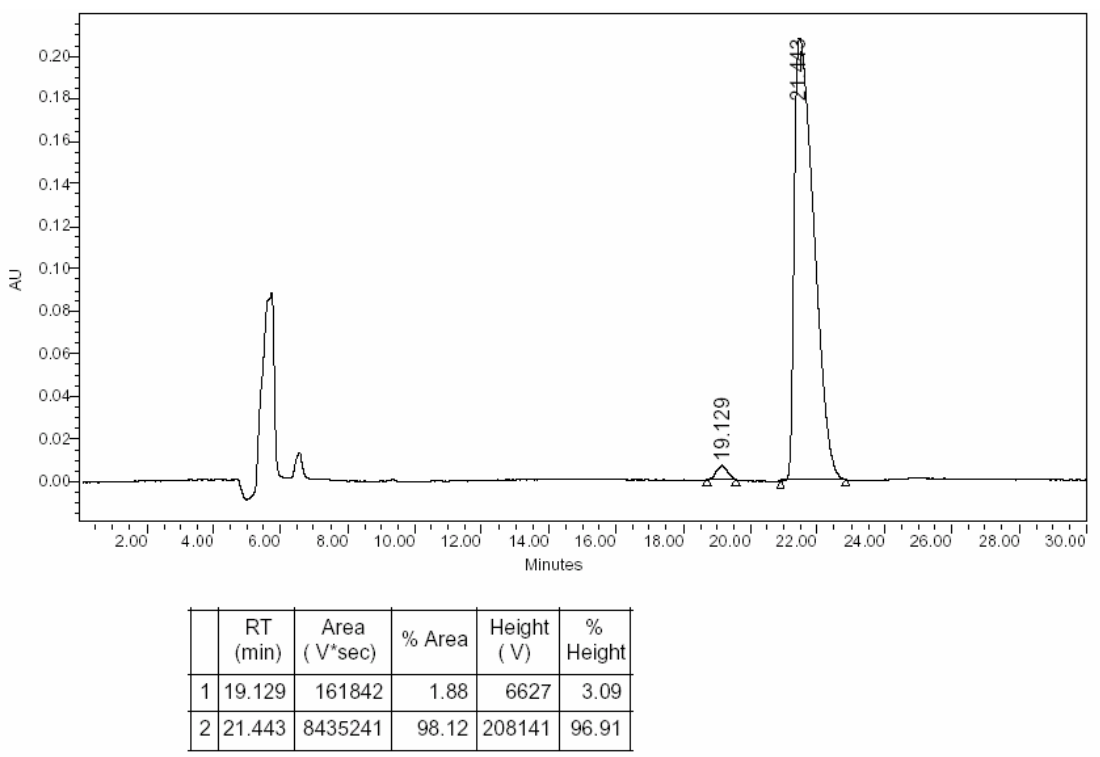

Table 2 entry 3

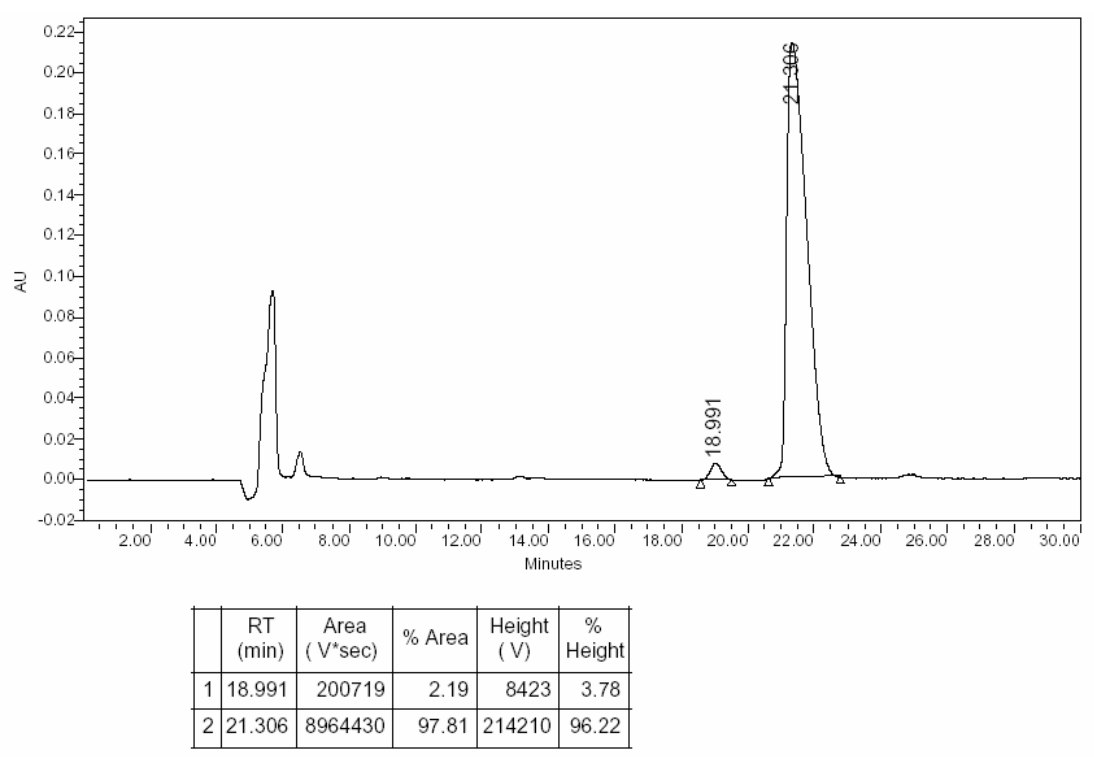


Table 2 entry 4

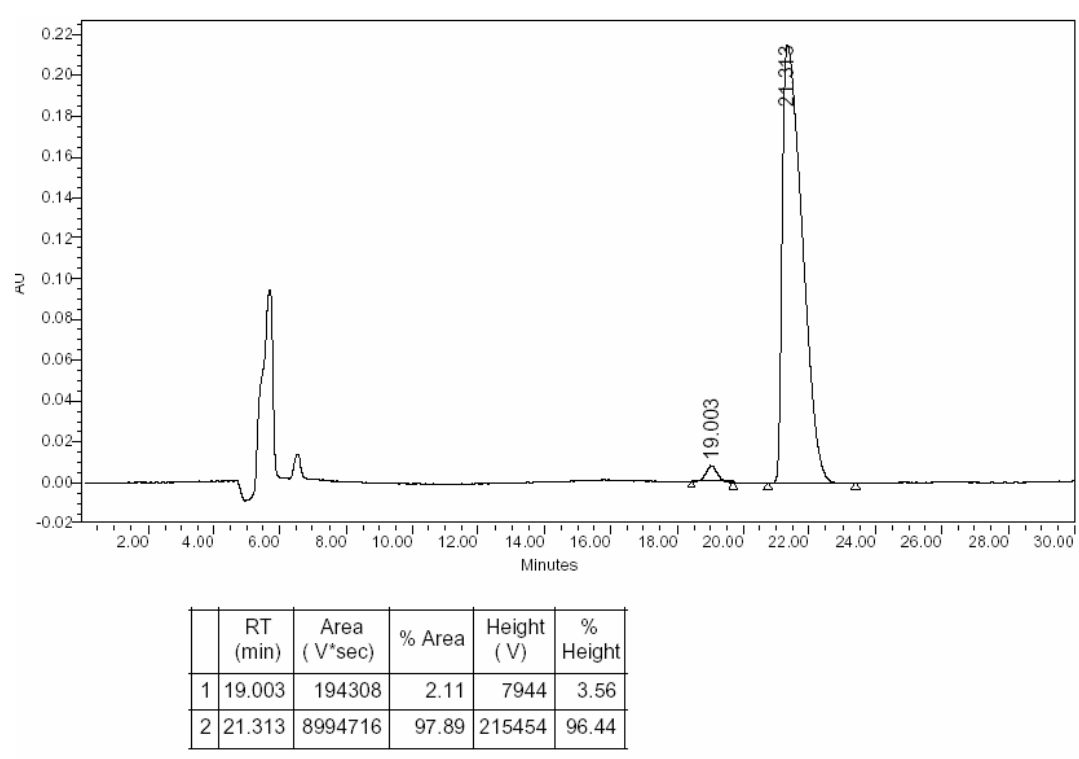

Table 2 entry 5

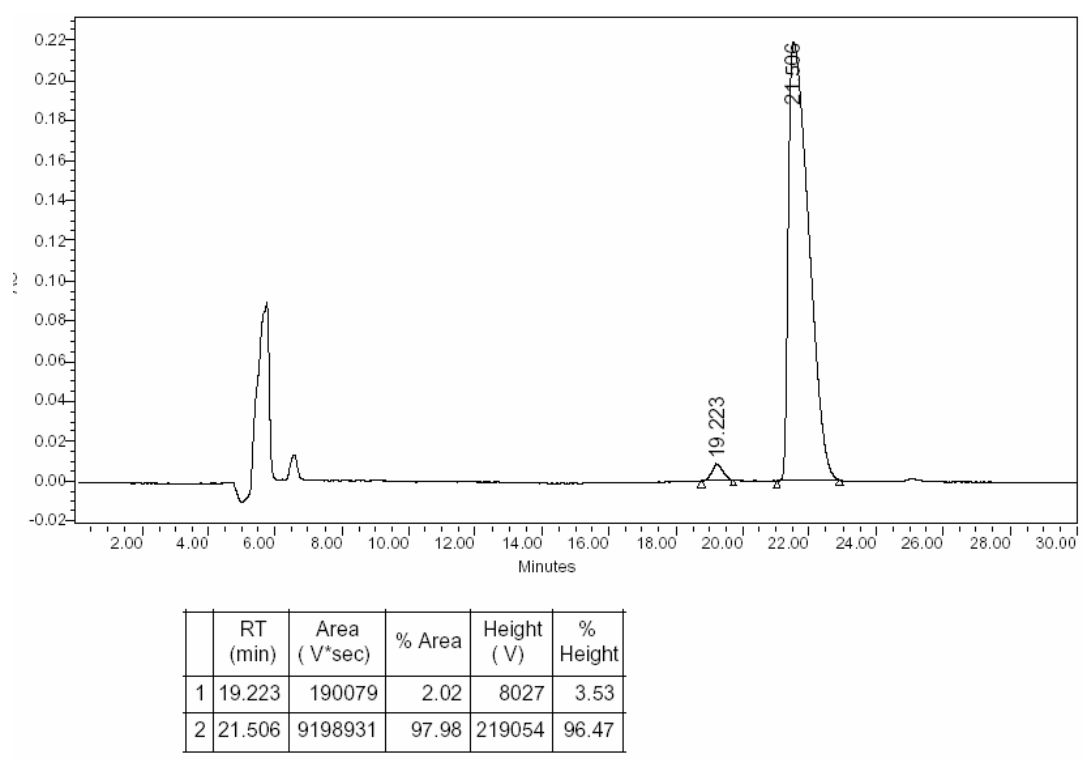


Table 2 entry 6

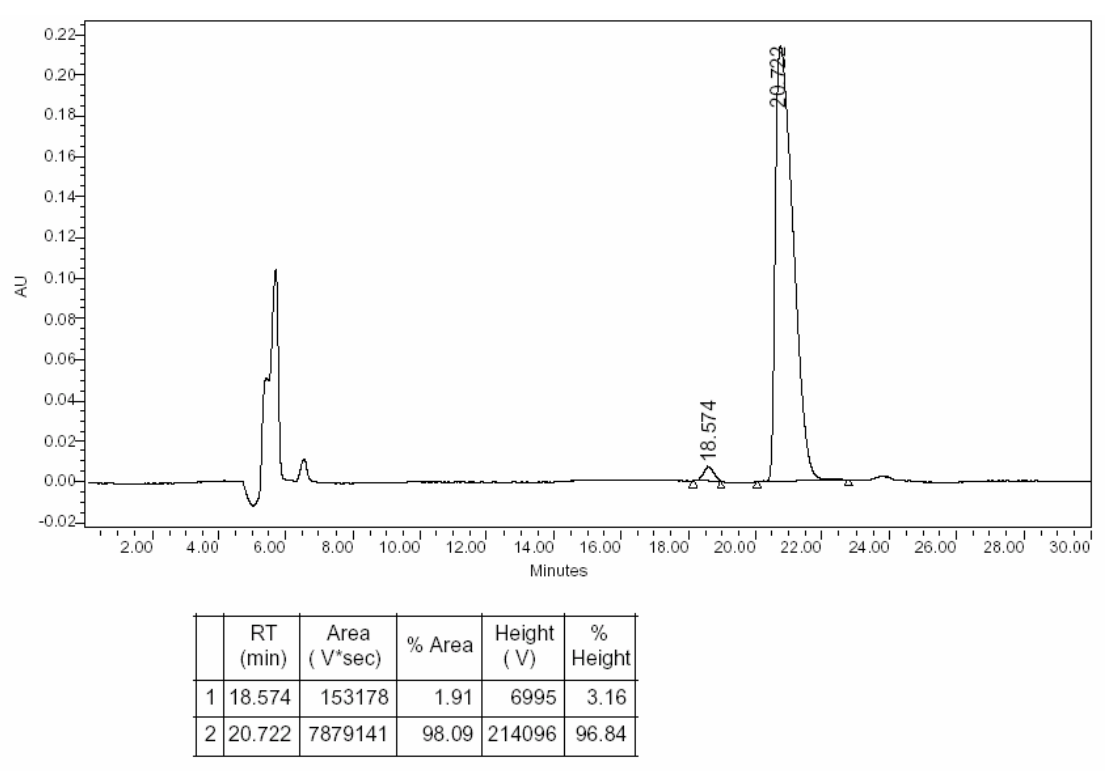

Table 2 entry 7

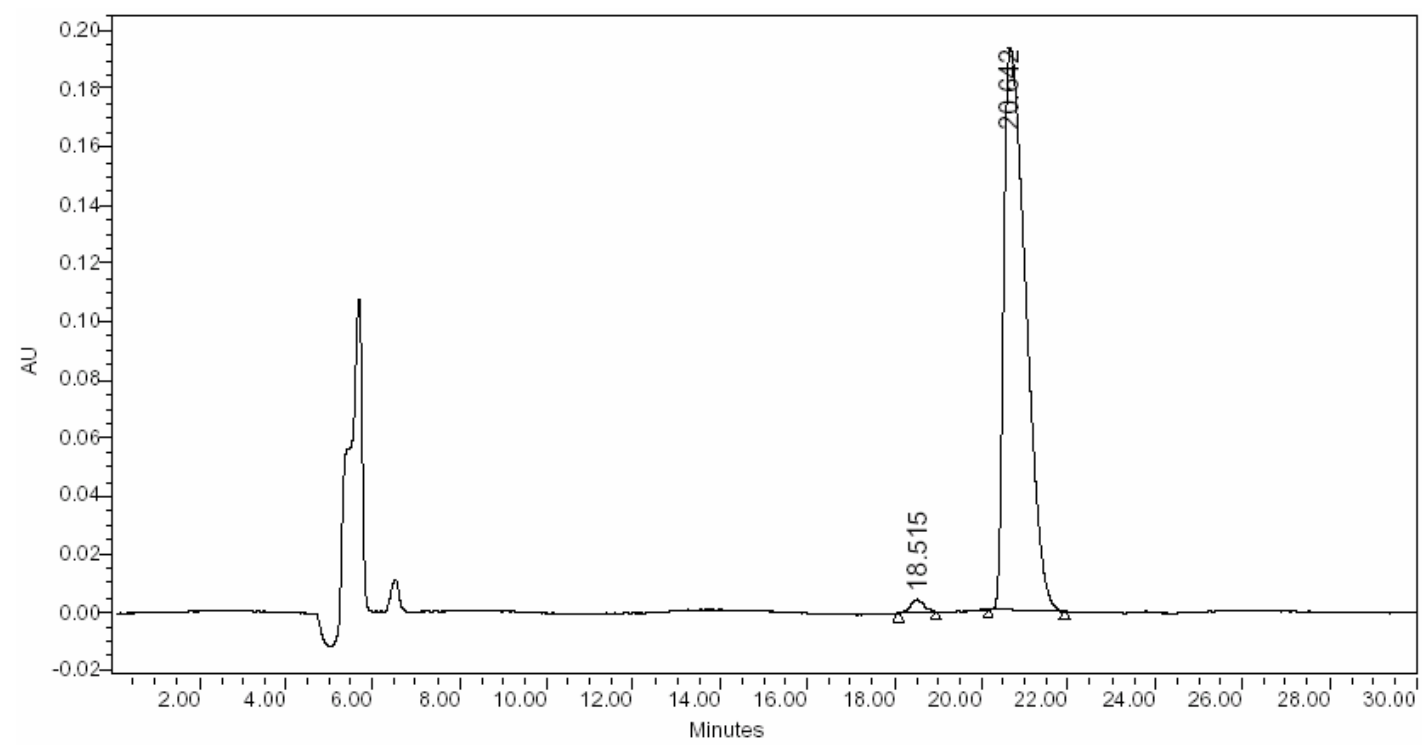

\begin{tabular}{|c|c|r|r|r|r|}
\hline & $\begin{array}{c}\text { RT } \\
(\mathrm{min})\end{array}$ & $\begin{array}{c}\text { Area } \\
\left(\mathrm{V}^{*} \mathrm{sec}\right)\end{array}$ & \% Area & $\begin{array}{c}\text { Height } \\
(\mathrm{V})\end{array}$ & $\begin{array}{c}\% \\
\text { Height }\end{array}$ \\
\hline 1 & 18.515 & 101199 & 1.46 & 4437 & 2.24 \\
\hline 2 & 20.642 & 6811485 & 98.54 & 193763 & 97.76 \\
\hline
\end{tabular}


Table 2 entry 8

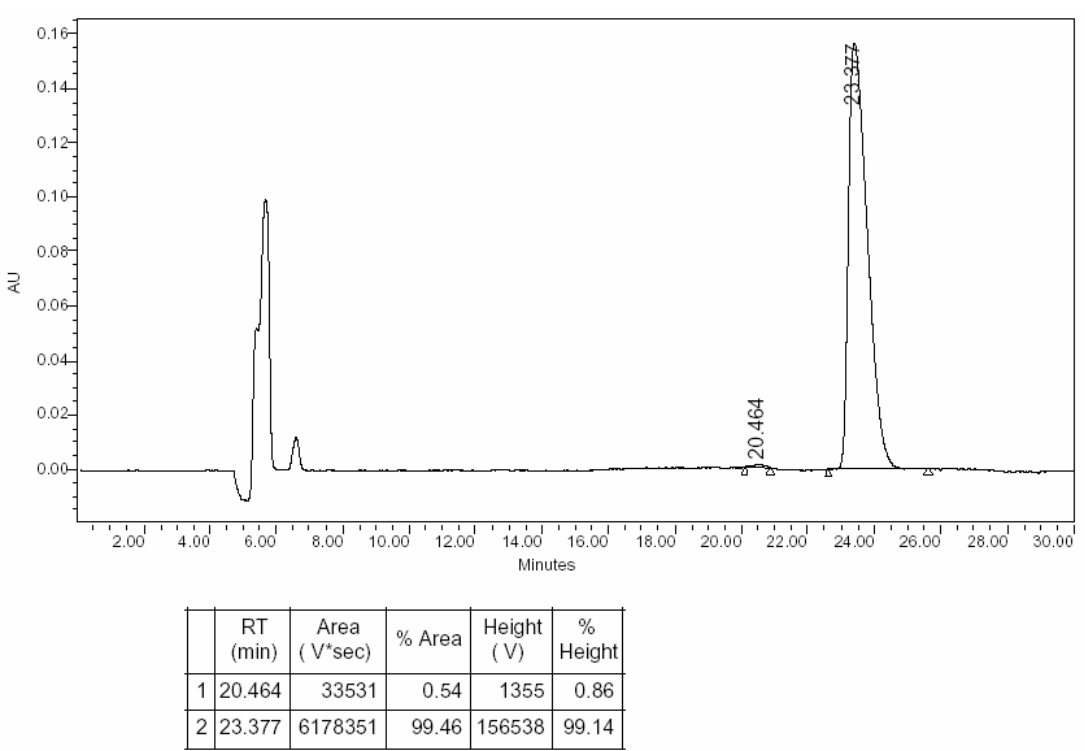

Table 2 entry 9

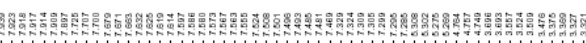

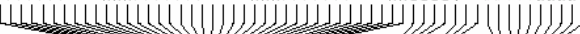
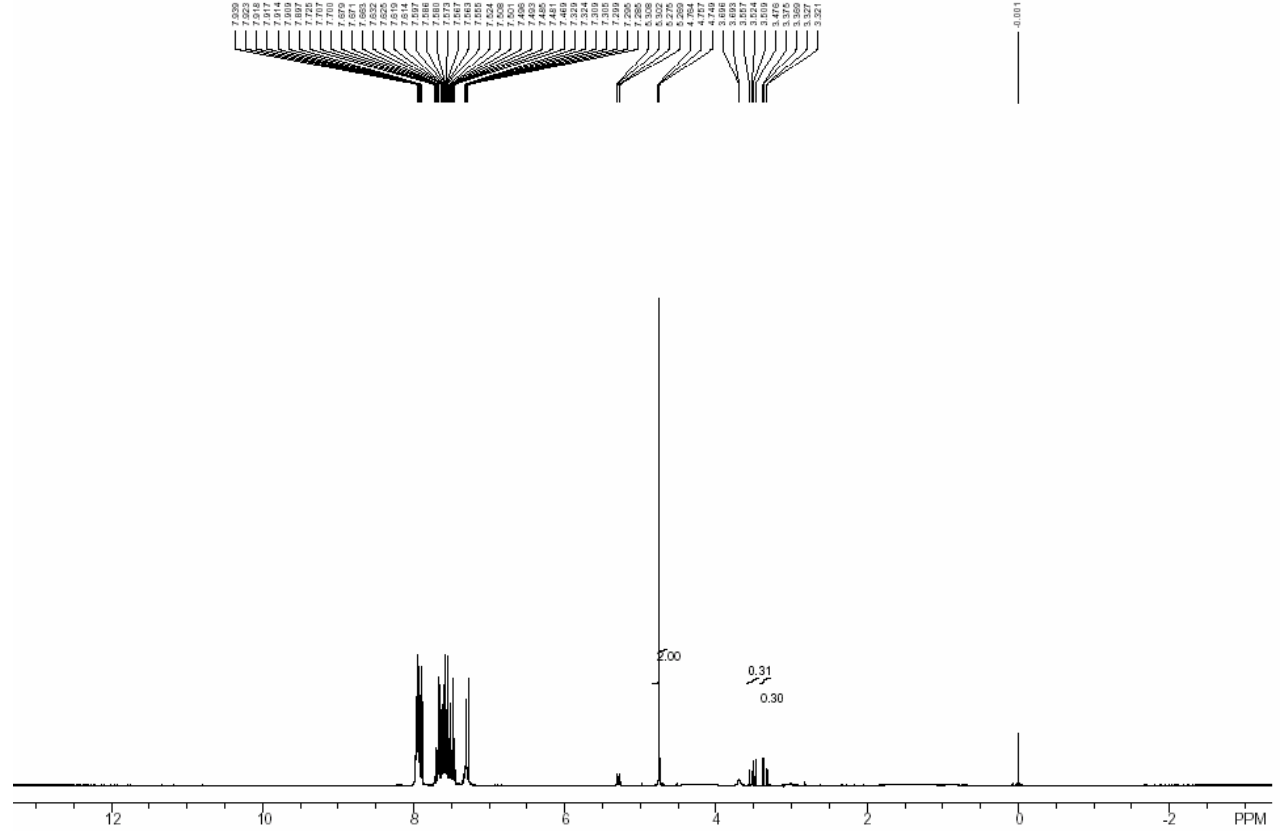


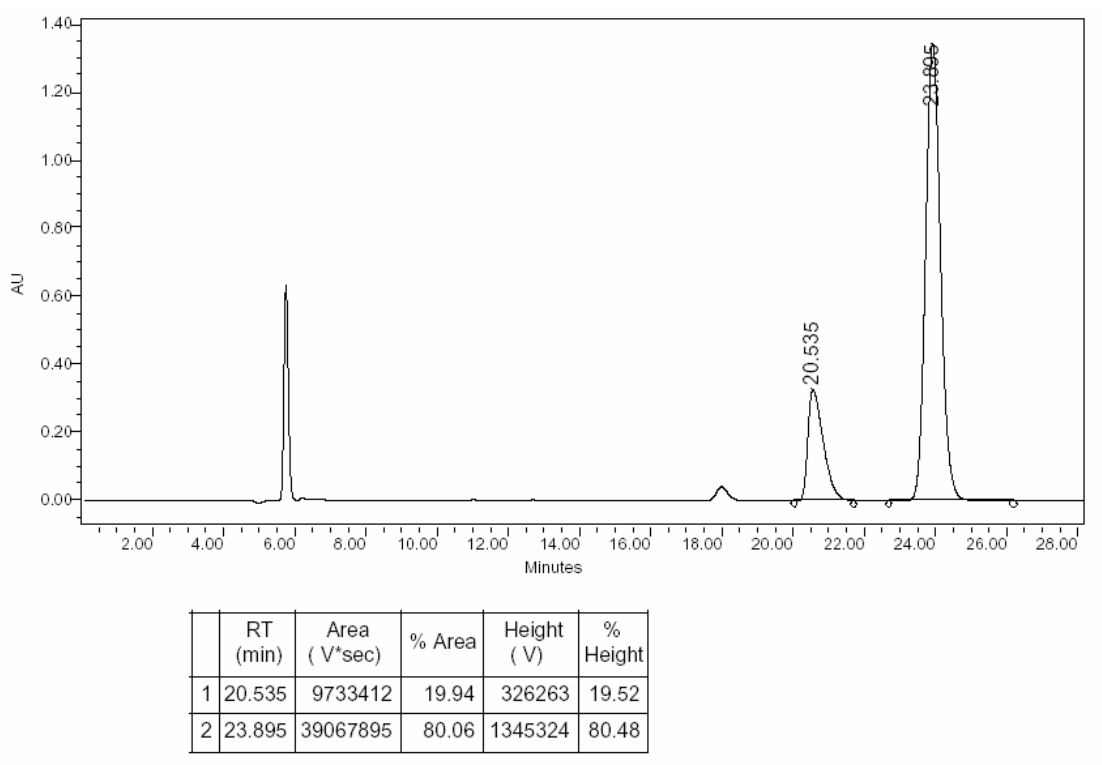

Table 2 entry 10

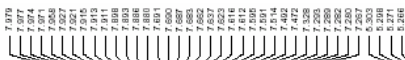
LLLLLULU

$11 / \frac{\pi}{\pi}$

Nifi
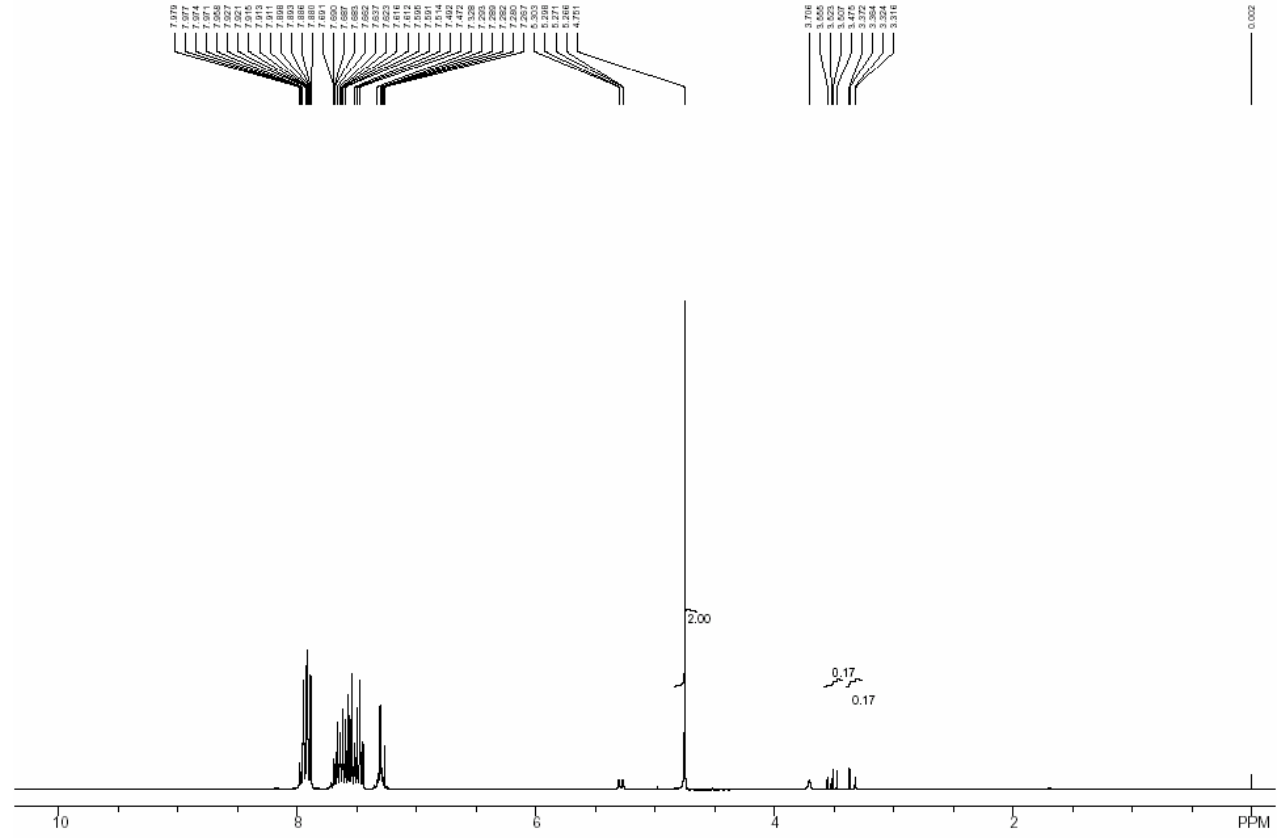


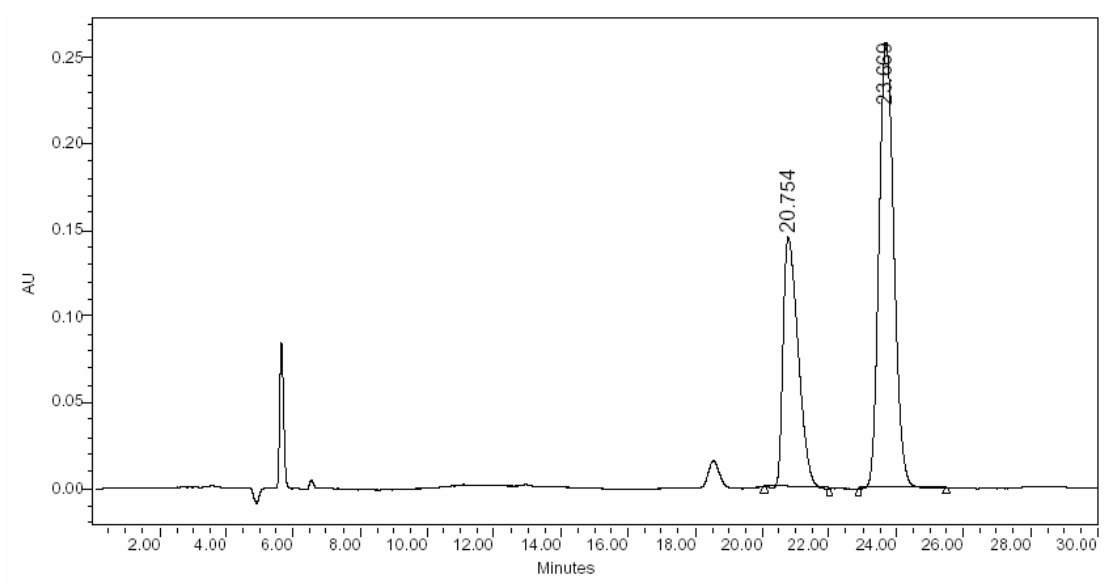

\begin{tabular}{|c|c|c|c|c|c|}
\hline & $\begin{array}{c}\text { RT } \\
(\mathrm{min})\end{array}$ & $\begin{array}{c}\text { Area } \\
\left(\mathrm{V}^{*} \mathrm{sec}\right)\end{array}$ & $\%$ Area & $\begin{array}{c}\text { Height } \\
(\mathrm{V})\end{array}$ & $\begin{array}{c}\% \\
\text { Height }\end{array}$ \\
\hline 1 & 20.754 & 4525468 & 36.17 & 144668 & 35.87 \\
\hline 2 & 23.669 & 7987692 & 63.83 & 258628 & 64.13 \\
\hline
\end{tabular}

Table 2 entry 11

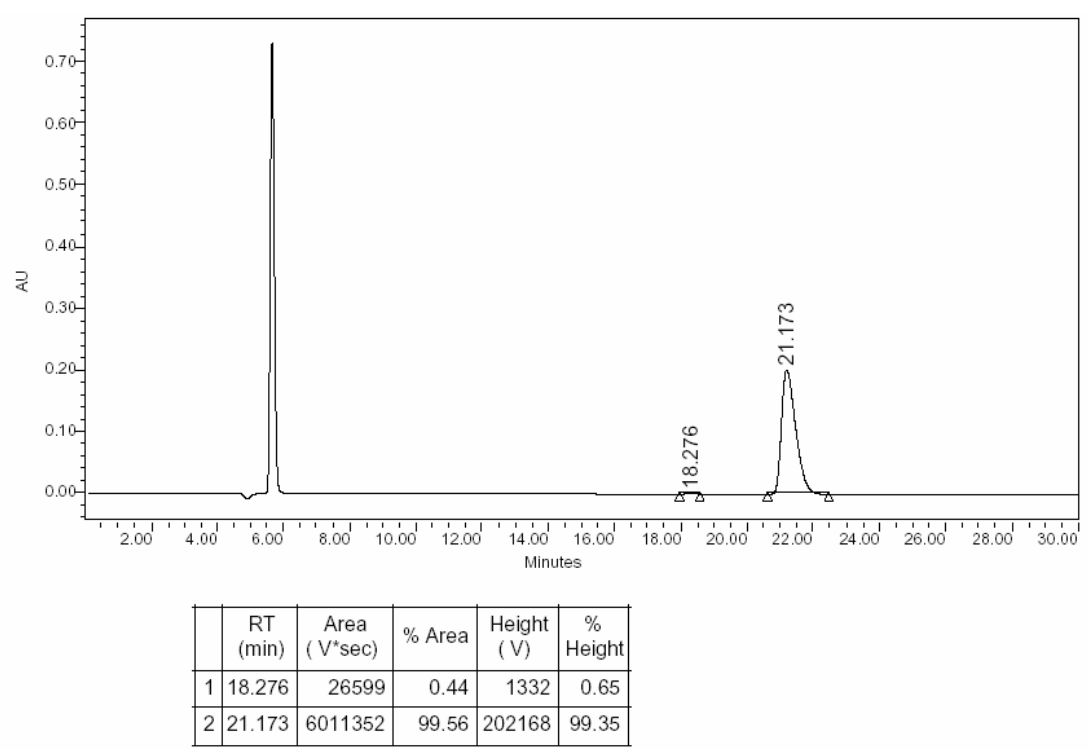


Table 2 entry 12

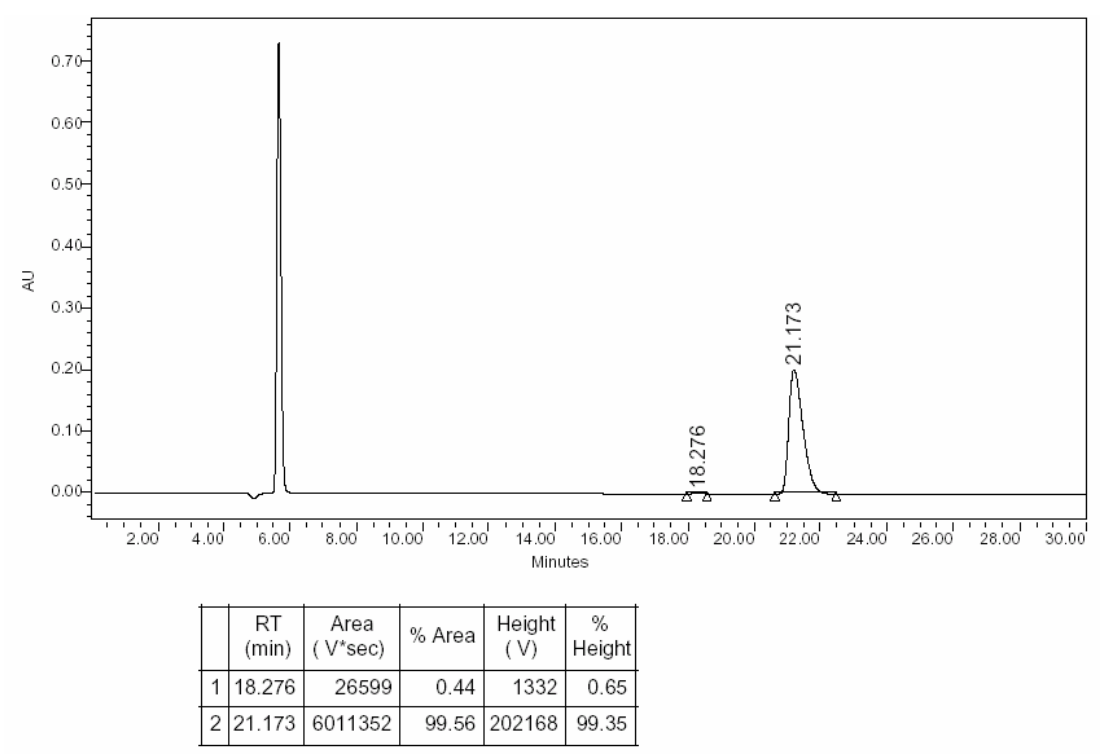

Table 2 entry 13

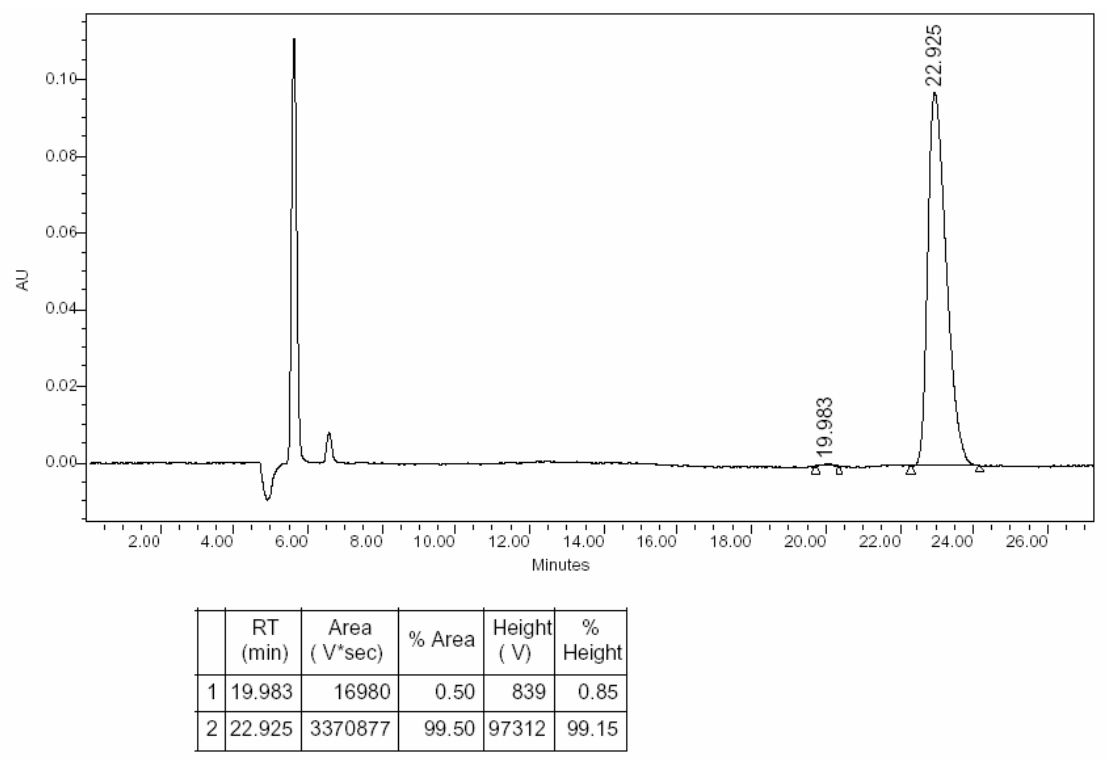




\section{HPLC and NMR data of Table3}<smiles>O=S(=O)(CC(O)c1ccccc1)c1ccccc1</smiles>

\section{$1 b$ racemate}

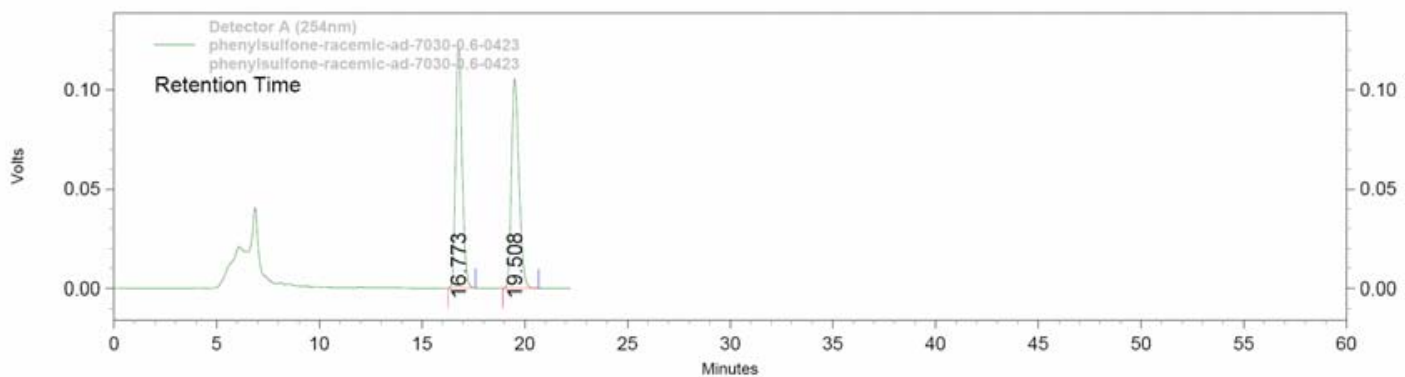

\begin{tabular}{rrrrrr}
\multicolumn{2}{c}{ Detector A } & $(254 n m)$ \\
Pk\# & Retention Time & Area & Area \% & Height & Height \% \\
\hline 1 & 16.773 & 2663411 & 49.915 & 123241 & 53.897 \\
2 & 19.508 & 2672507 & 50.085 & 105418 & 46.103
\end{tabular}

Table 3 entry 1

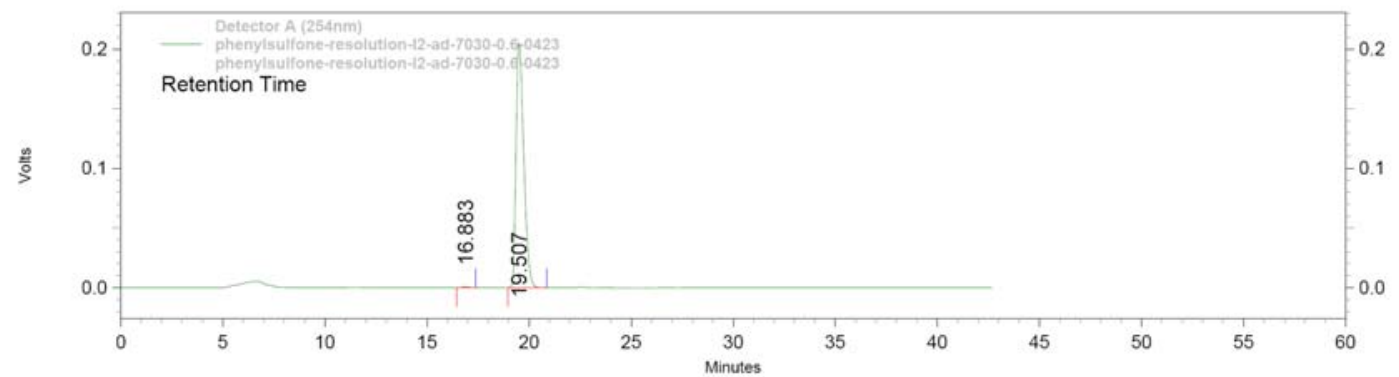

Detector A (254nm)

\begin{tabular}{rrrrrr} 
Pk\# & Retention Time & Area & Area \% & Height & Height \% \\
\hline 1 & 16.883 & 19452 & 0.357 & 839 & 0.407 \\
2 & 19.507 & 5432477 & 99.643 & 205257 & 99.593
\end{tabular}


<smiles>O=S(=O)(CC(O)c1ccc(F)cc1)c1ccccc1</smiles>

\section{2b racemate}

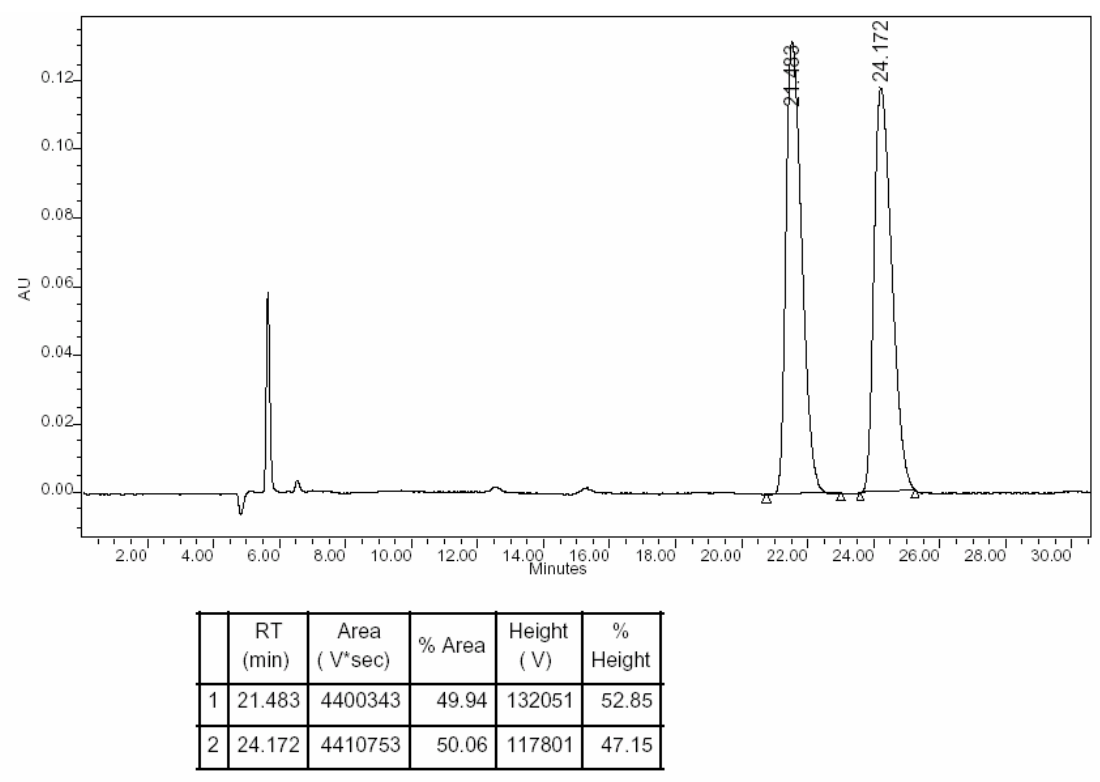

Table 3 entry 2

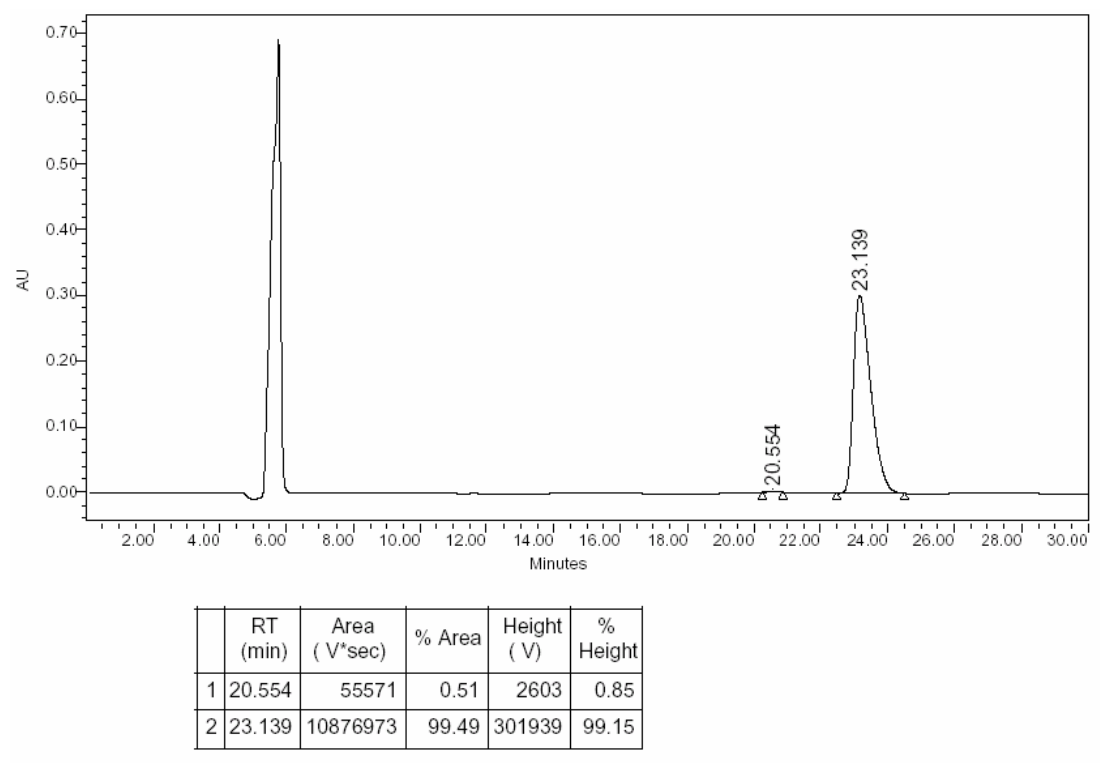


<smiles>O=S(=O)(CC(O)c1ccc(Cl)cc1)c1ccccc1</smiles>

\section{$2 c$ racemate}

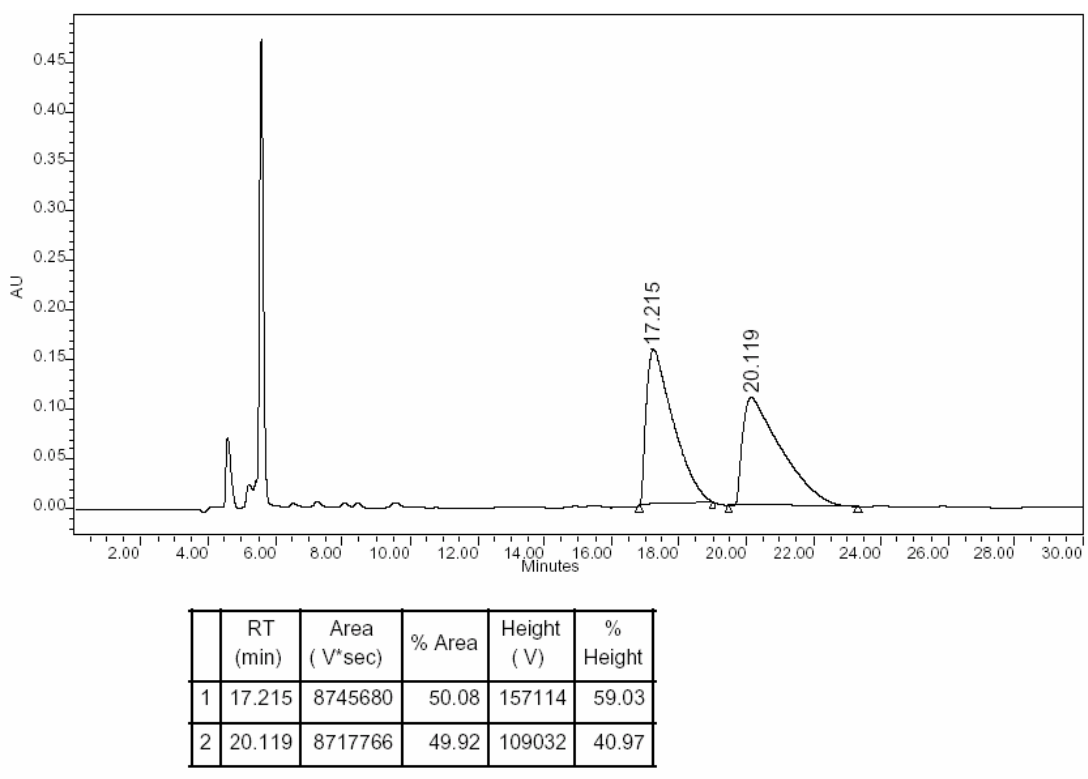

Table 3 entry 3

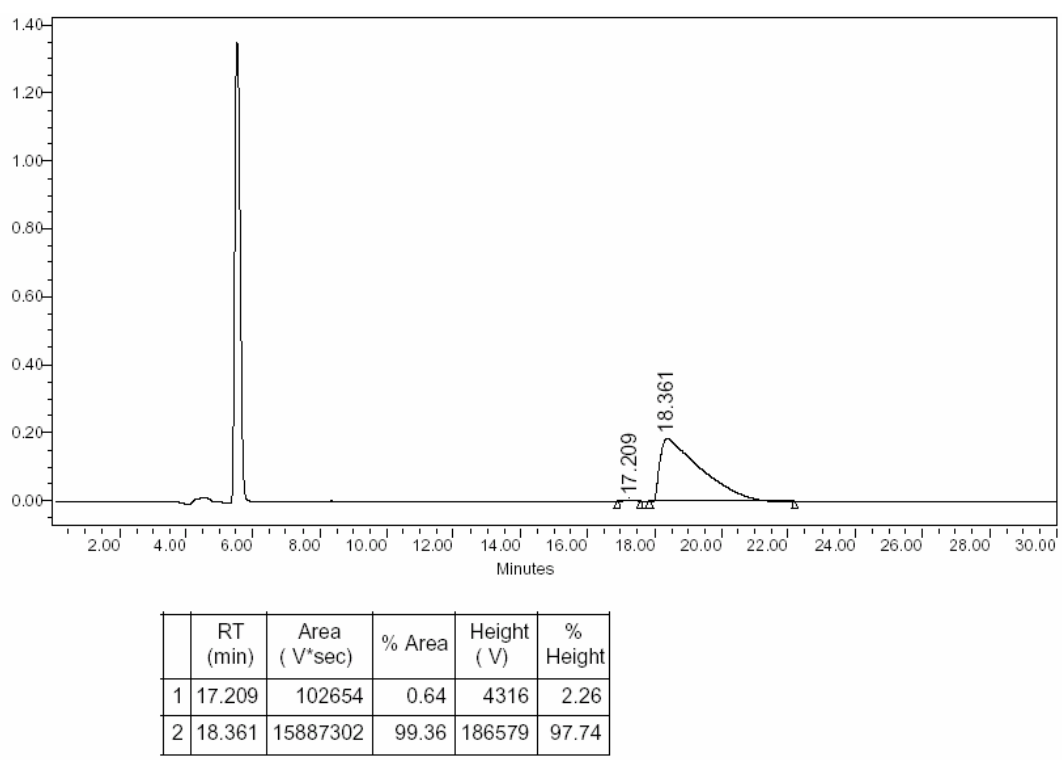


<smiles>O=S(=O)(CC(O)c1ccc(Br)cc1)c1ccccc1</smiles>

\section{$2 d$ racemate}

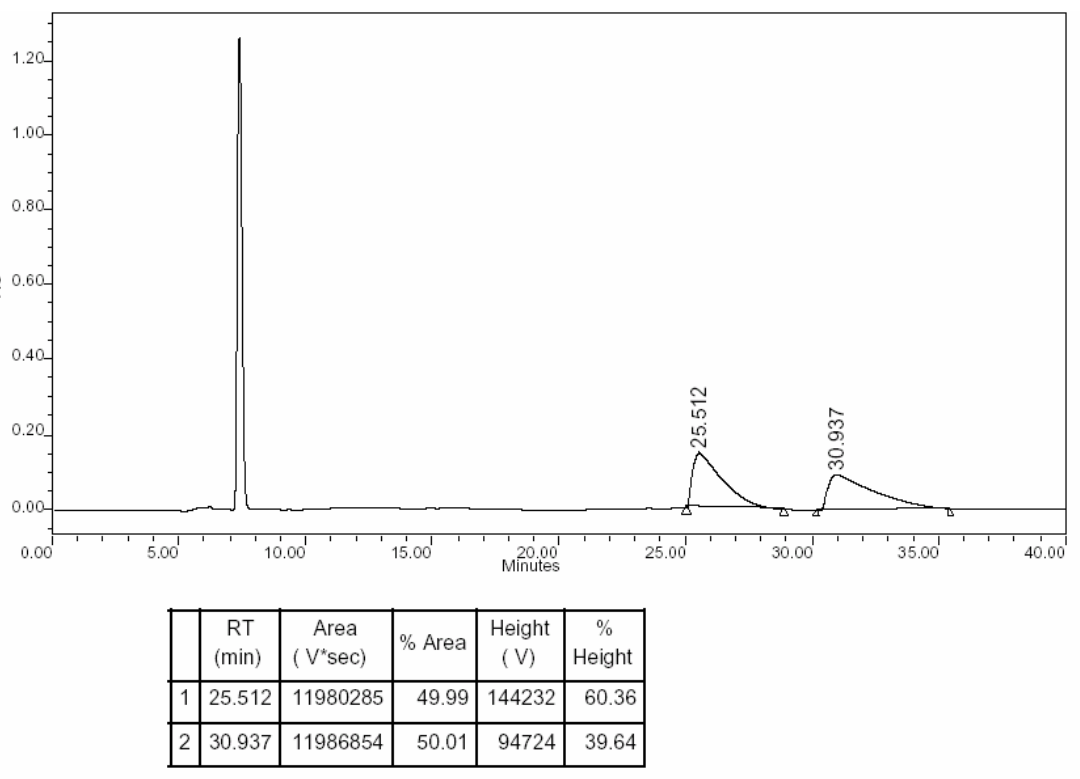

Table 3 entry 4

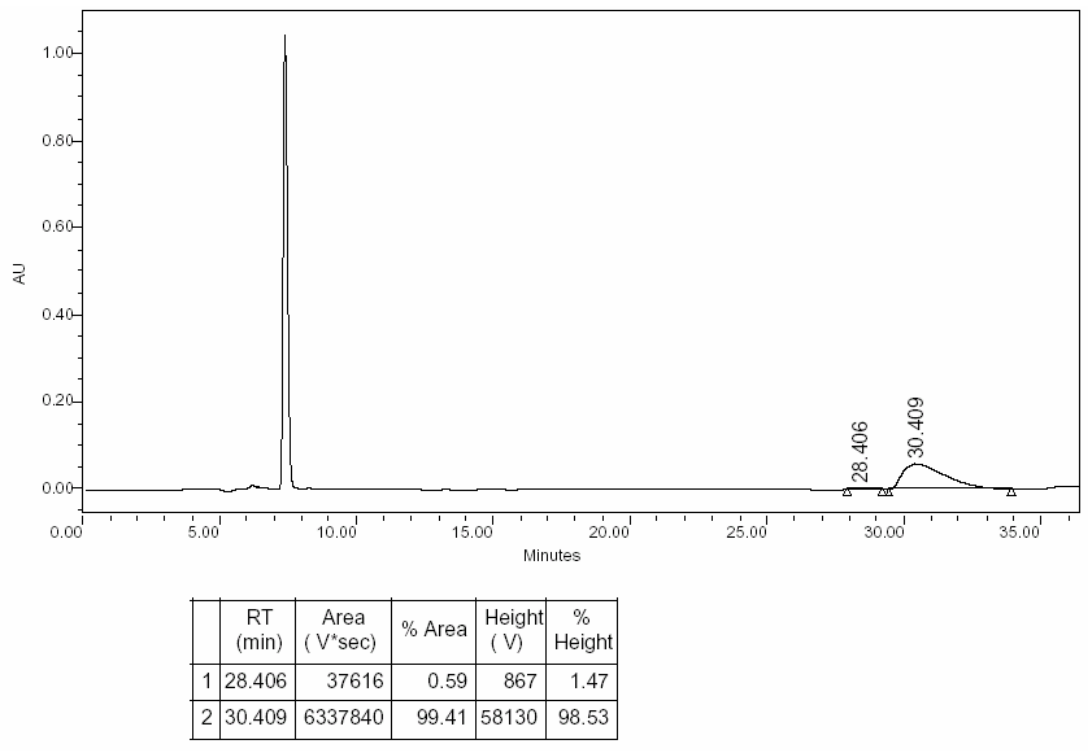




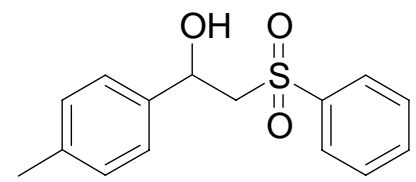

2e racemate

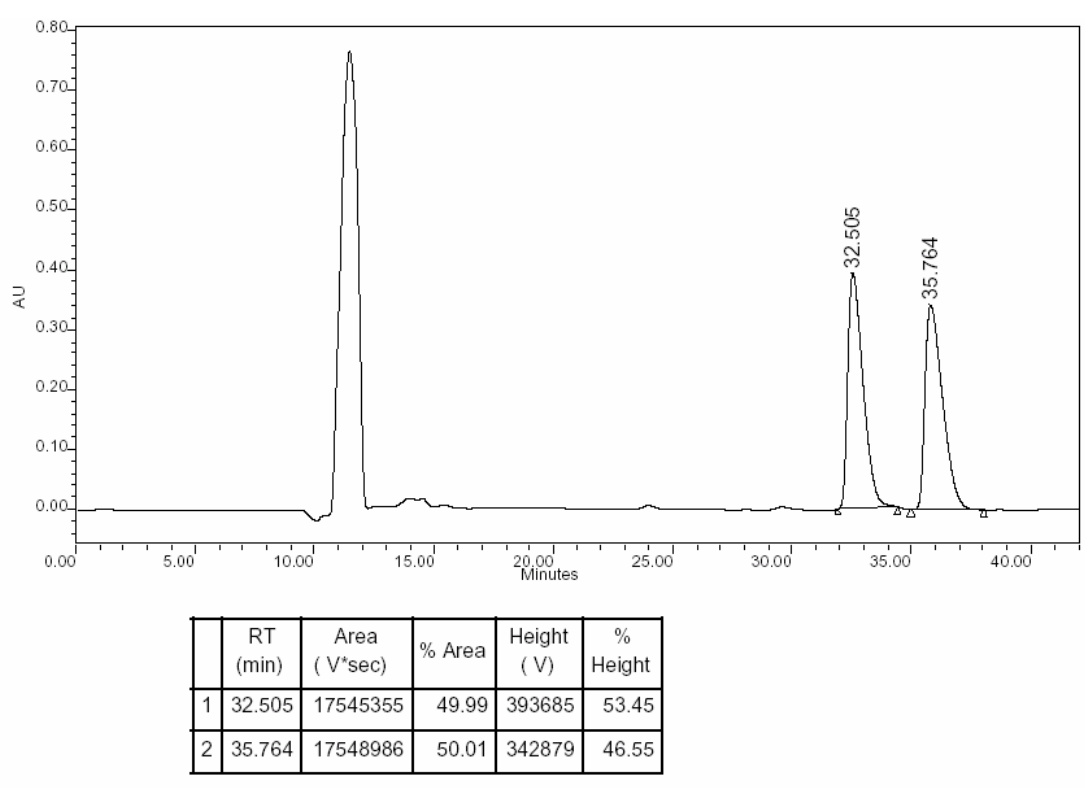

Table 3 entry 5

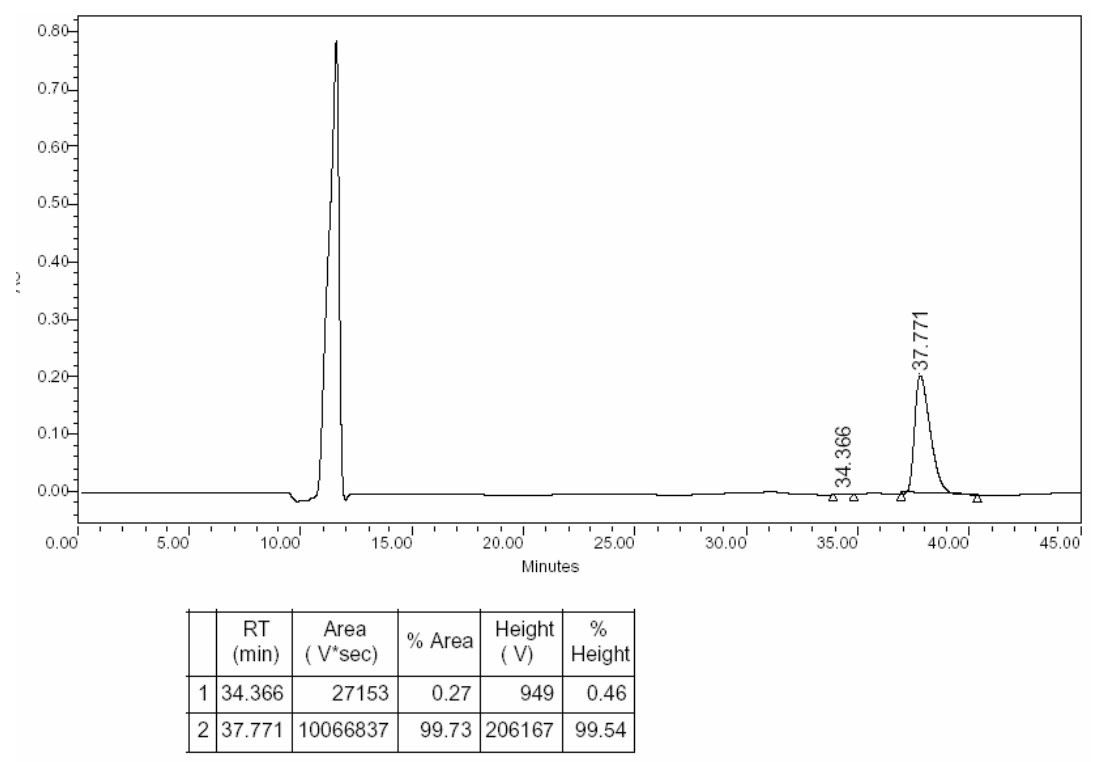


<smiles>COc1ccc(C(O)CS(=O)(=O)c2ccccc2)cc1</smiles>

\section{2f racemate}

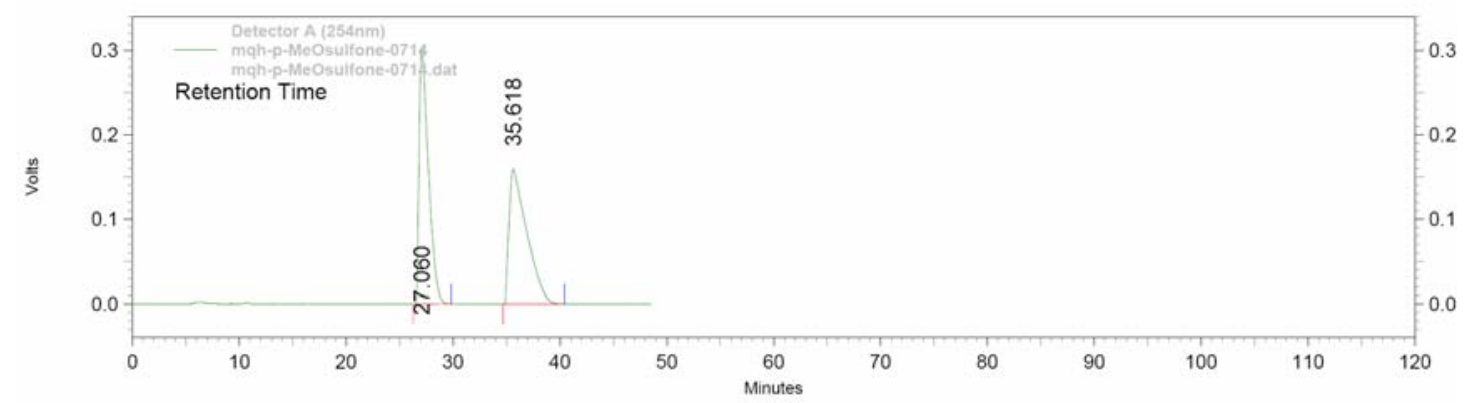

Detector A (254nm)

\begin{tabular}{rrrrrr} 
Pk\# & Retention Time & Area & Area \% & Height & Height \% \\
\hline 1 & 27.060 & 18370261 & 49.958 & 302273 & 65.447 \\
2 & 35.618 & 18401174 & 50.042 & 159585 & 34.553
\end{tabular}

\section{Table 3 entry 6}

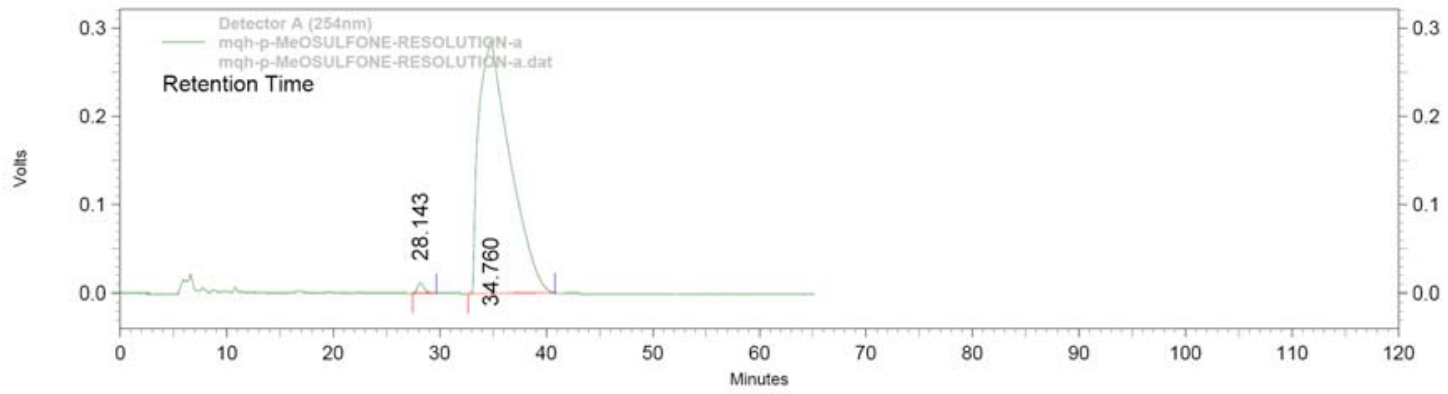

Detector A (254nm)

\begin{tabular}{|c|c|c|c|c|c|}
\hline Pk \# & Retention Time & Area & Area $\%$ & Height & Height $\%$ \\
\hline 1 & 28.143 & 498692 & 0.855 & 11374 & 3.837 \\
\hline 2 & 34.760 & 57811715 & 99.145 & 285039 & 96.163 \\
\hline
\end{tabular}


<smiles>O=S(=O)(CC(O)c1cccc(Cl)c1)c1ccccc1</smiles>

\section{2 g racemate}

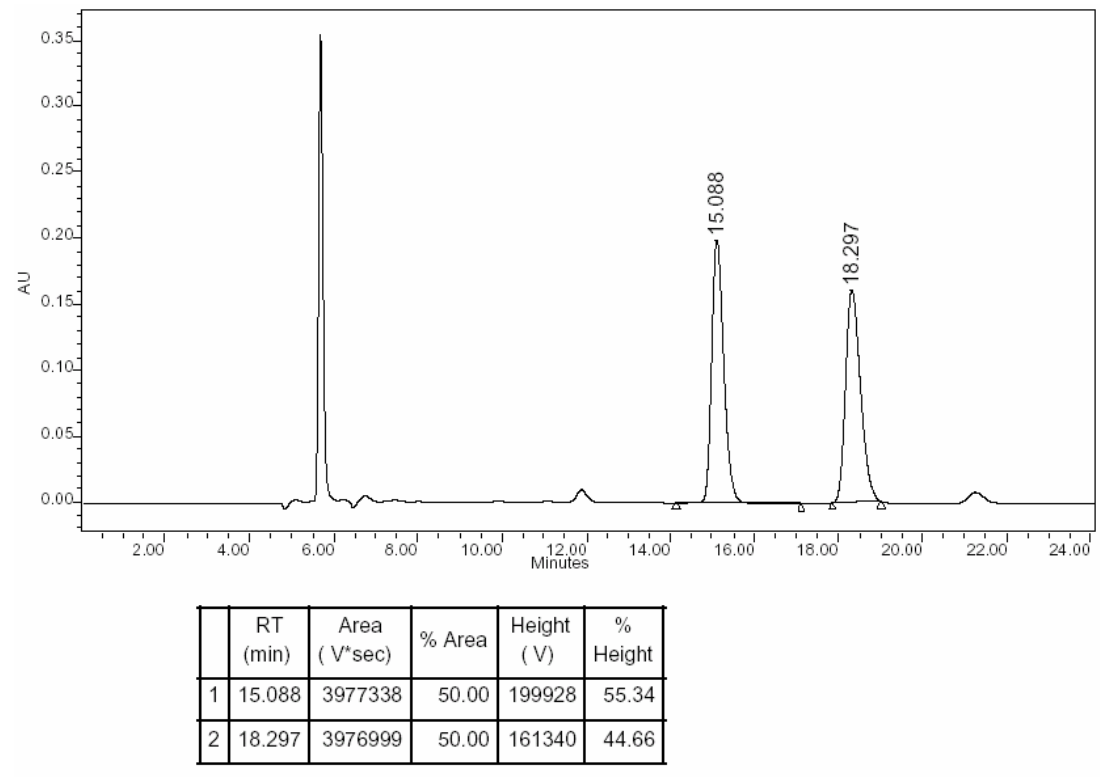

Table 3 entry 7

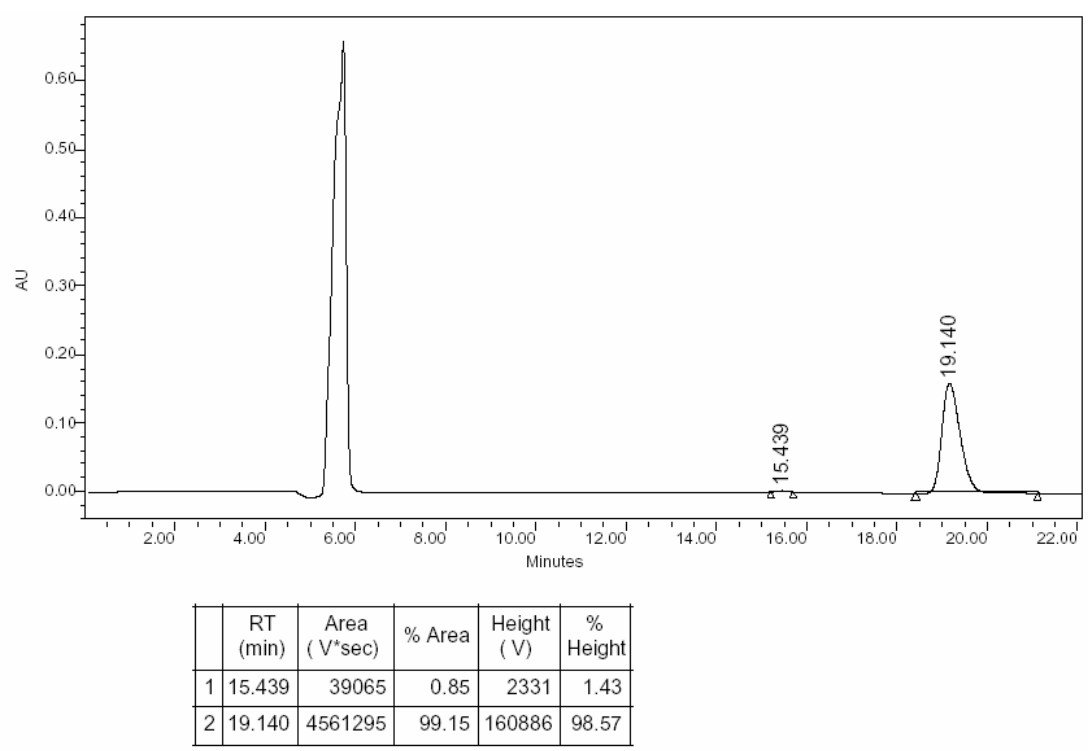


<smiles>Cc1ccccc1C(O)CS(=O)(=O)c1ccccc1</smiles>

\section{$2 h$ racemate}

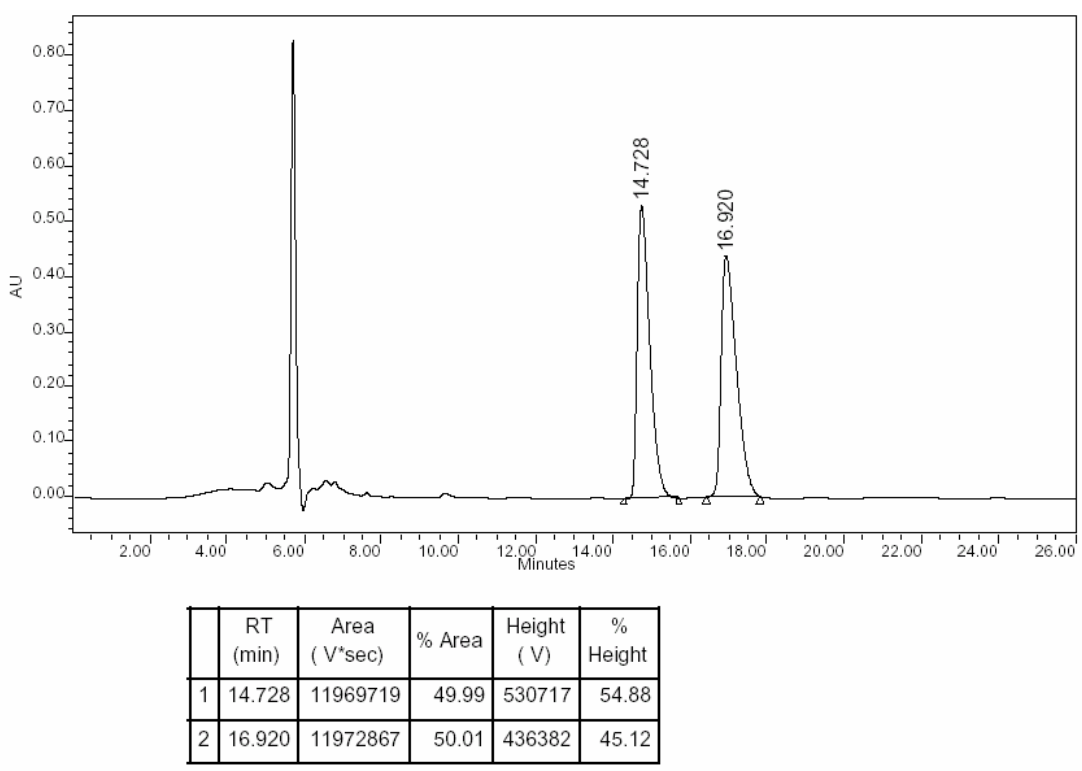

Table 3 entry 8

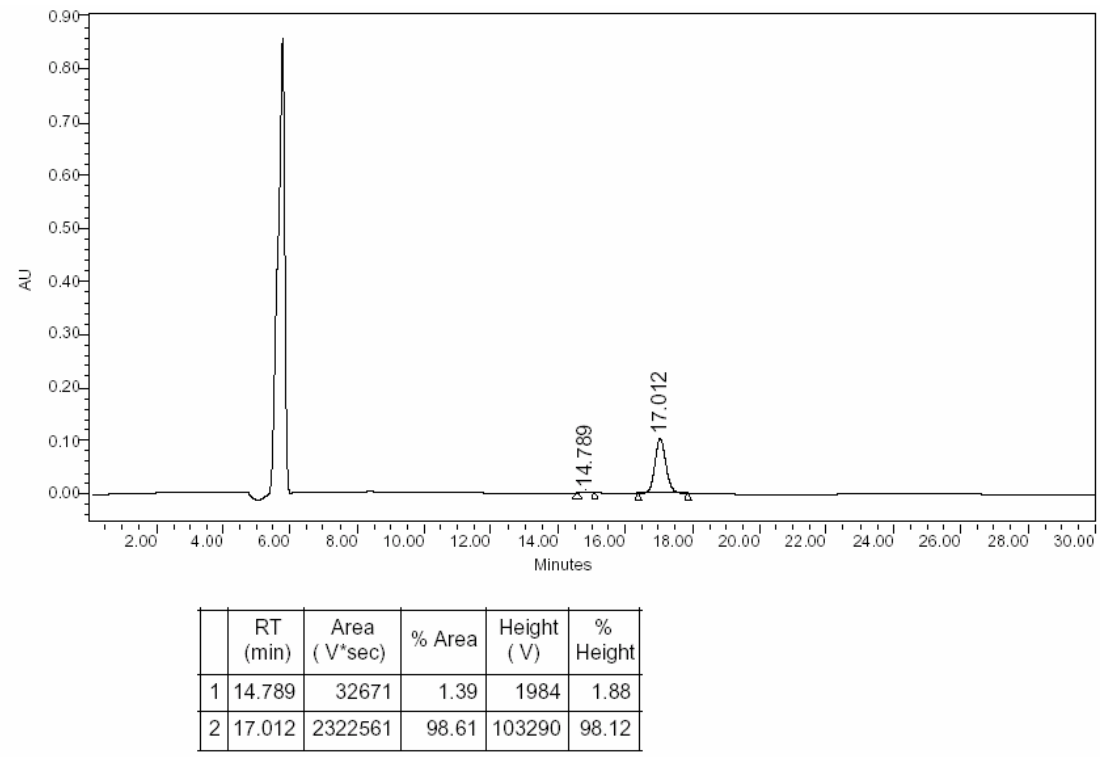




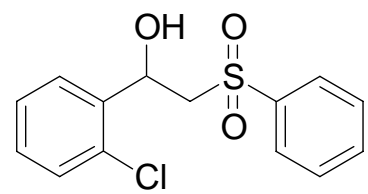

\section{$2 \mathbf{i}$ racemate}

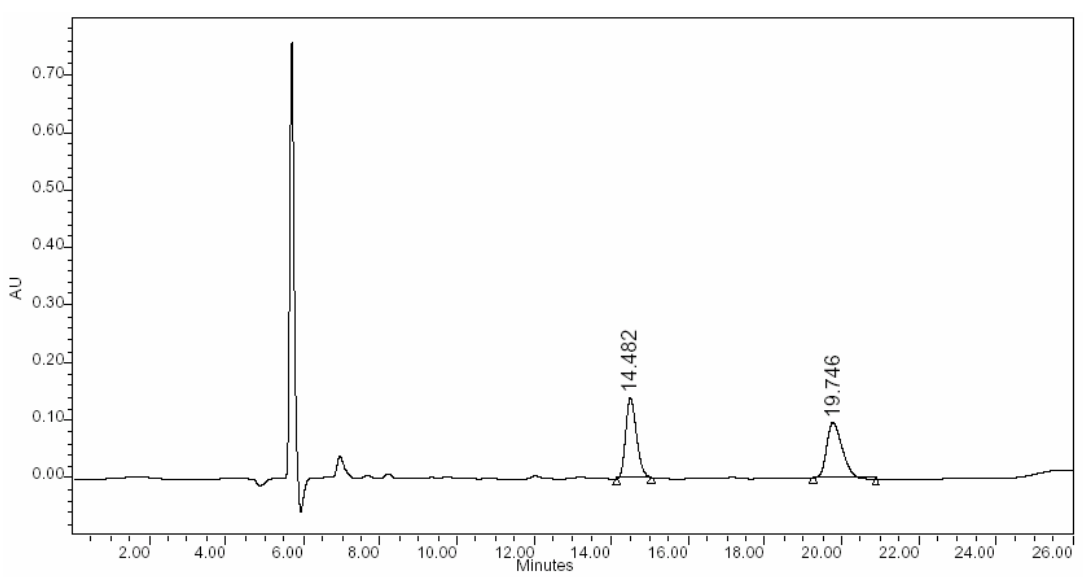

\begin{tabular}{|c|c|c|c|c|c|}
\hline & $\begin{array}{c}\mathrm{RT} \\
(\mathrm{min})\end{array}$ & $\begin{array}{c}\text { Area } \\
\left(\mathrm{V}^{*} \mathrm{sec}\right)\end{array}$ & $\%$ Area & $\begin{array}{c}\text { Height } \\
(\mathrm{V})\end{array}$ & $\begin{array}{c}\% \\
\text { Height }\end{array}$ \\
\hline 1 & 14.482 & 2704110 & 50.09 & 138699 & 59.13 \\
\hline 2 & 19.746 & 2694401 & 49.91 & 95874 & 40.87 \\
\hline
\end{tabular}

Table 3 entry 9

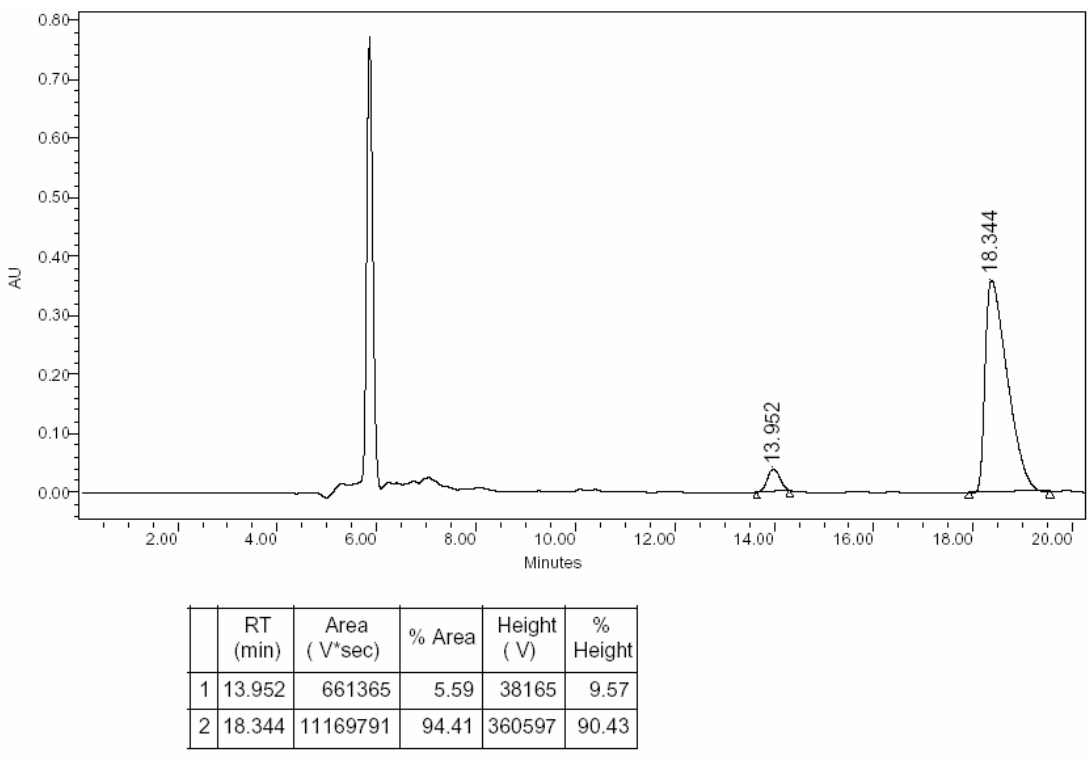




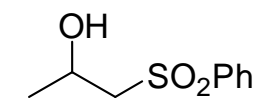

\section{$2 \mathrm{j}$ racemate}

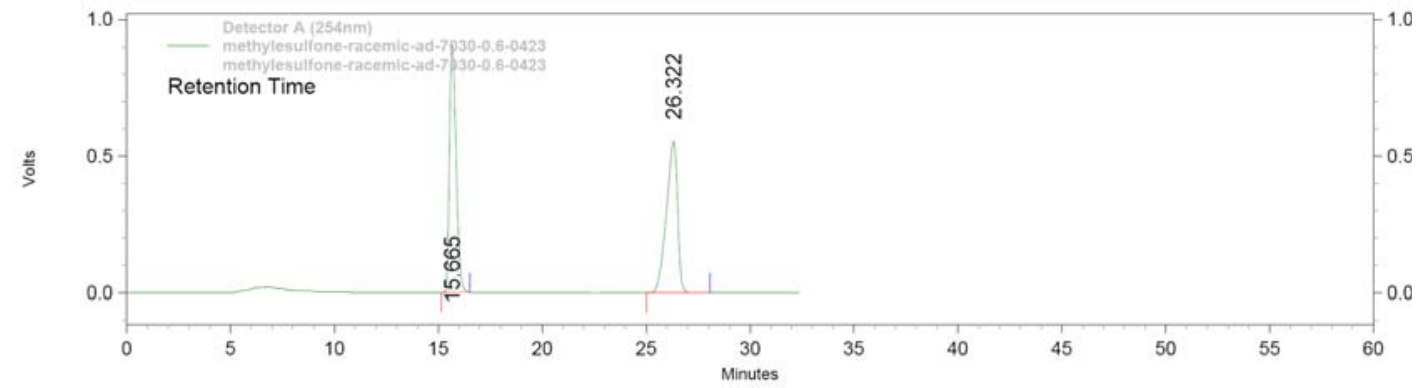

\begin{tabular}{|c|c|c|c|c|c|}
\hline Pl. \# & $\begin{array}{l}4 \mathbf{n m}) \\
\text { Pintion }\end{array}$ & Aneg & $4 \operatorname{sen} 0$ & Heint & Hoinhto \\
\hline 1 & 15.665 & 20132298 & 49.374 & 906856 & 62.056 \\
\hline 2 & 26.322 & 20642516 & 50.626 & 554487 & 37.944 \\
\hline
\end{tabular}

Table 3 entry 10

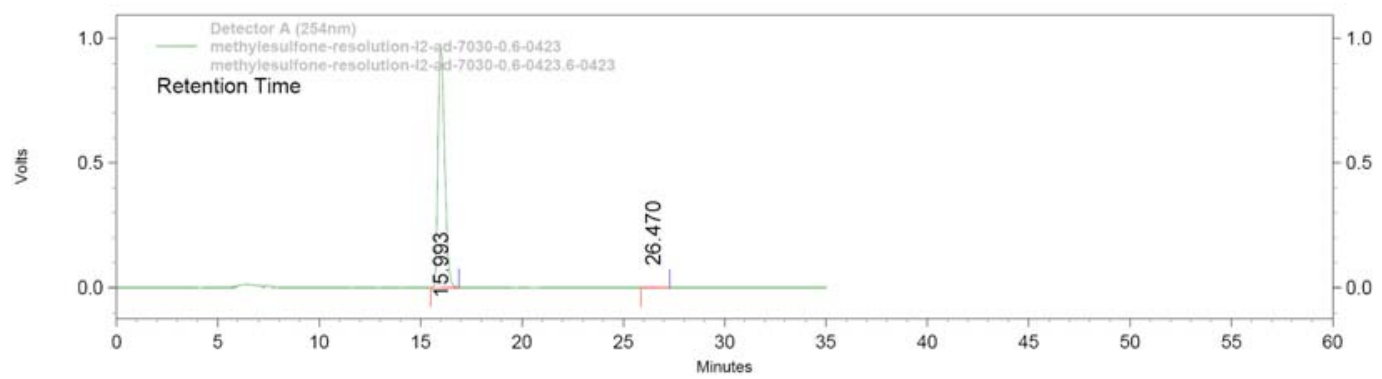

Detector A (254nm)

\begin{tabular}{rrrrrr} 
Pk\# & Retention Time & Area & Area \% & Height & Height \% \\
\hline 1 & 15.993 & 22095713 & 99.719 & 971066 & 99.802 \\
2 & 26.470 & 62339 & 0.281 & 1925 & 0.198
\end{tabular}


<smiles>CC(C)C(O)CS(=O)(=O)c1ccccc1</smiles>

\section{$2 k$ racemate}

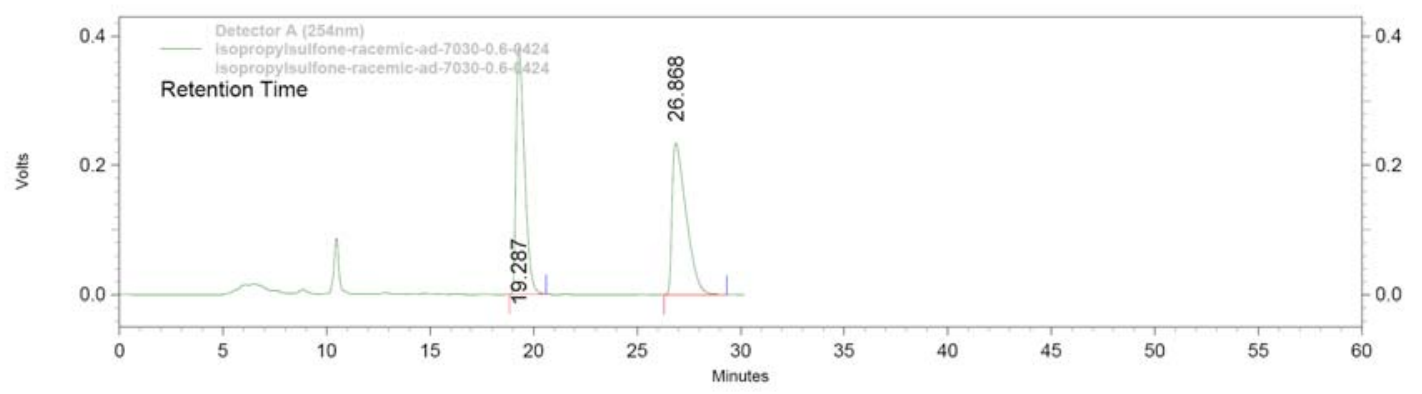

\begin{tabular}{|c|c|c|c|c|c|}
\hline \multicolumn{6}{|c|}{ Detector A (254nm) } \\
\hline Pk\# & Retention Time & Area & Area \% & Height & Height $\%$ \\
\hline 1 & 19.287 & 10852563 & 49.667 & 381921 & 61.972 \\
\hline 2 & 26.868 & 10997980 & 50.333 & 234362 & 38.028 \\
\hline
\end{tabular}

\section{Table 3 entry 11}

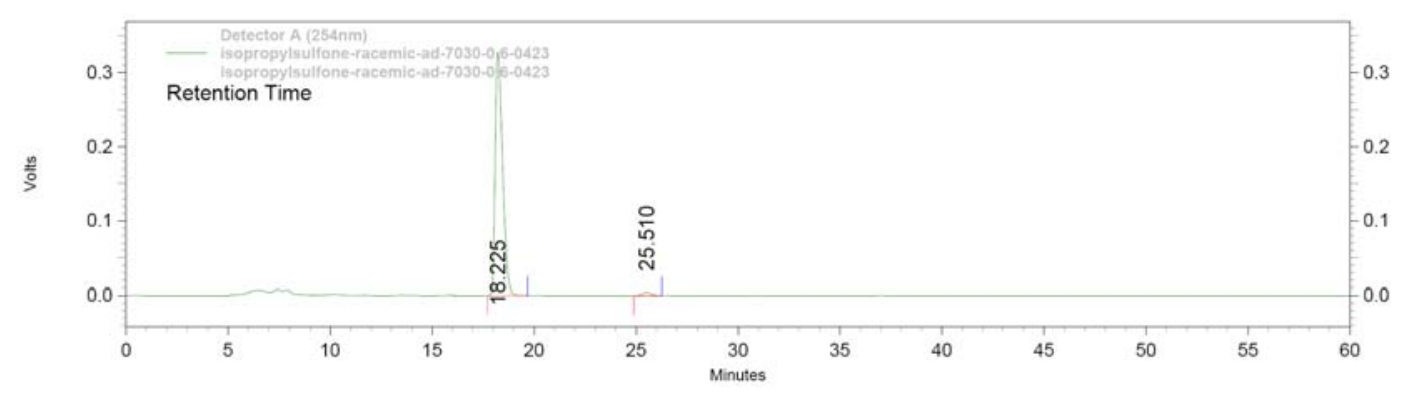

Detector A (254nm)

\begin{tabular}{rrrrrr} 
Pk \# & Retention Time & Area & Area \% & Height & Height \% \\
\hline 1 & 18.225 & 8279509 & 98.481 & 327888 & 98.762 \\
2 & 25.510 & 127748 & 1.519 & 4111 & 1.238
\end{tabular}


<smiles>O=S(=O)(CC(O)C1CCCCC1)c1ccccc1</smiles>

\section{2 l racemate}

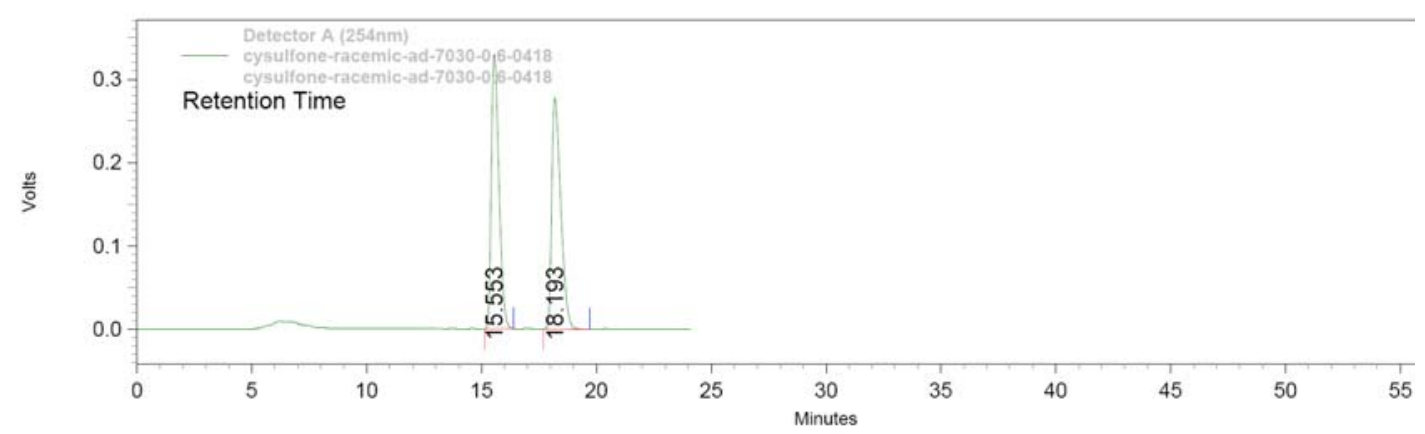

Detector A (254nm)

\begin{tabular}{rrrrrr} 
Pk\# & Retention Time & Area & Area \% & Height & Height \% \\
\hline 1 & 15.553 & 7553766 & 49.804 & 329336 & 54.277 \\
2 & 18.193 & 7613303 & 50.196 & 277428 & 45.723
\end{tabular}

Table 3 entry 12

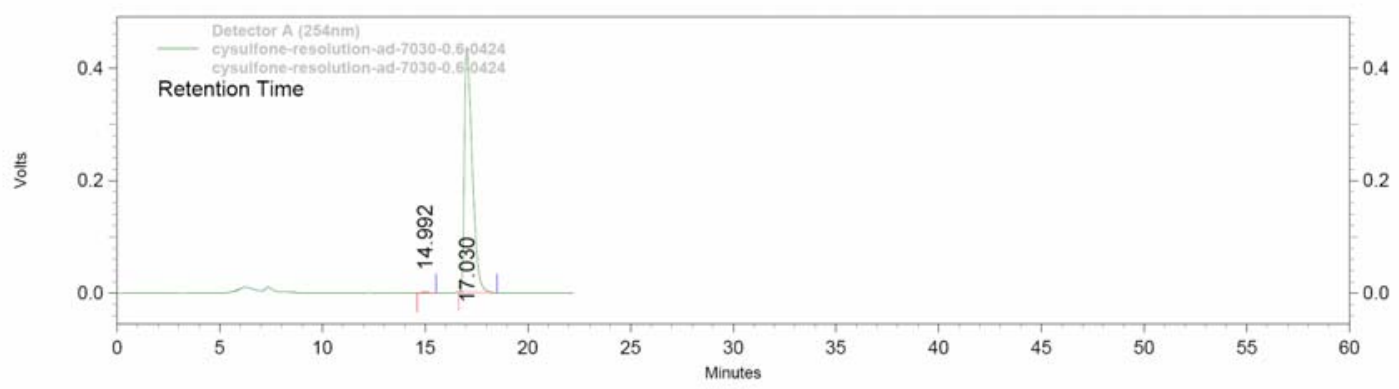

Detector A (254nm)

\begin{tabular}{rrrrrr} 
Pk\# & Retention Time & Area & Area \% & Height & Height \% \\
\hline 1 & 14.992 & 41310 & 0.357 & 2194 & 0.502 \\
2 & 17.030 & 11530693 & 99.643 & 435119 & 99.498
\end{tabular}




\section{Table 3 entry 13}
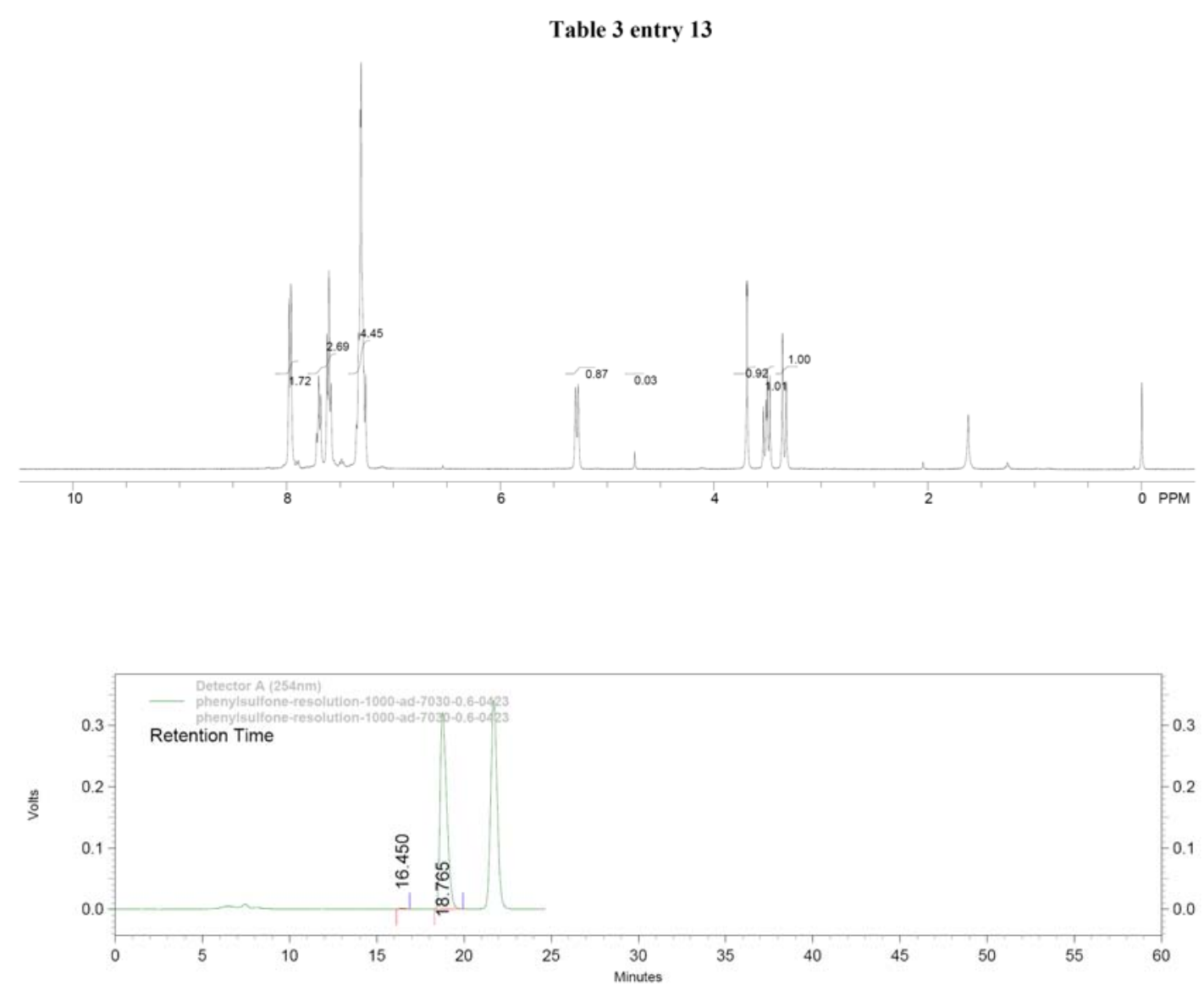

Detector A (254nm)

\begin{tabular}{rrrrrr}
\multicolumn{1}{c}{ Pk\# } & Retention Time & Area & Area \% & Height & Height \% \\
\hline 1 & 16.450 & 36998 & 0.435 & 1683 & 0.522 \\
2 & 18.765 & 8466190 & 99.565 & 320779 & 99.478
\end{tabular}




\section{HPLC and NMR data of Table4}

\section{Table 4 entry 1}

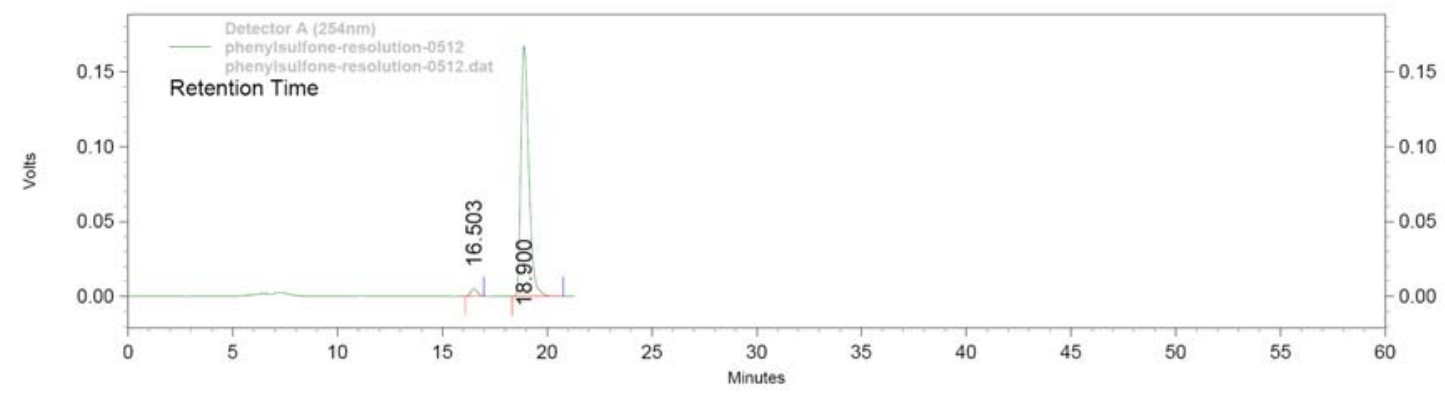

Detector A (254nm)

\begin{tabular}{rrrrrr} 
Pk\# & Retention Time & Area & Area \% & Height & Height \% \\
\hline 1 & 16.503 & 106566 & 2.361 & 4942 & 2.865 \\
2 & 18.900 & 4406677 & 97.639 & 167549 & 97.135
\end{tabular}

Table 4 entry 2

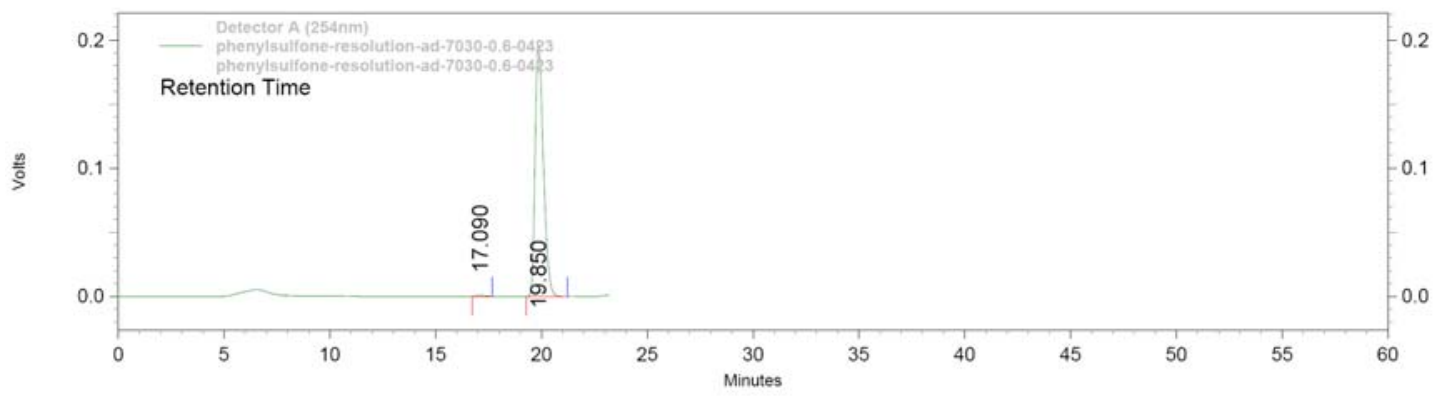

Detector A (254nm)

\begin{tabular}{rrrrrr} 
Pk\# & Retention Time & Area & Area \% & Height & Height \% \\
\hline 1 & 17.090 & 24133 & 0.452 & 1039 & 0.526 \\
2 & 19.850 & 5316932 & 99.548 & 196358 & 99.474
\end{tabular}


Table 4 entry 3

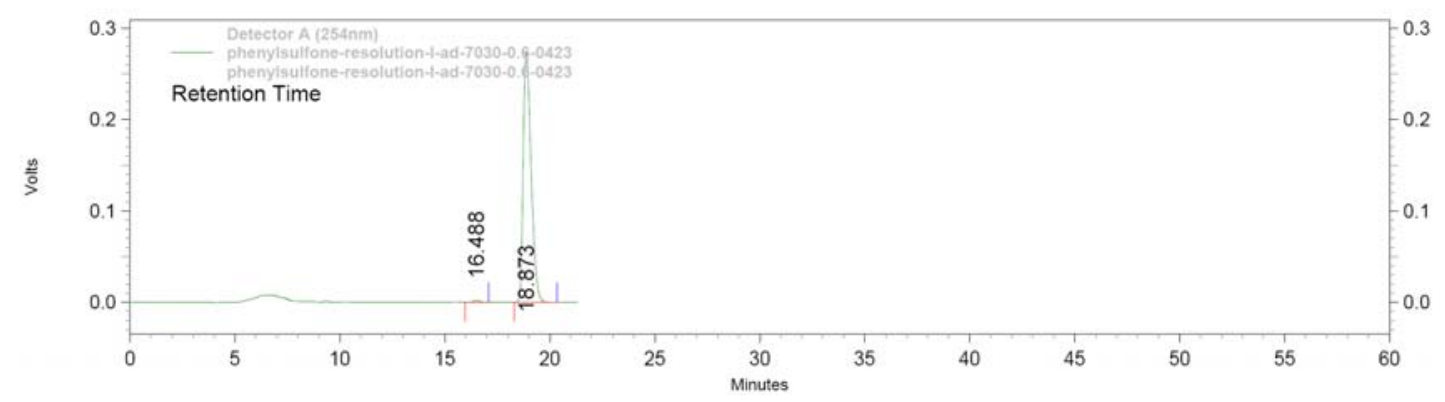

Detector A (254nm)

Pk\# Retention Time

Area

Area \%

Height

Height \%

\begin{tabular}{|c|c|c|c|c|c|}
\hline 1 & 16.488 & 43281 & 0.603 & 1968 & 0.711 \\
\hline 2 & 18.873 & 7134959 & 99.397 & 274637 & 99.289 \\
\hline
\end{tabular}

Table 4 entry 4

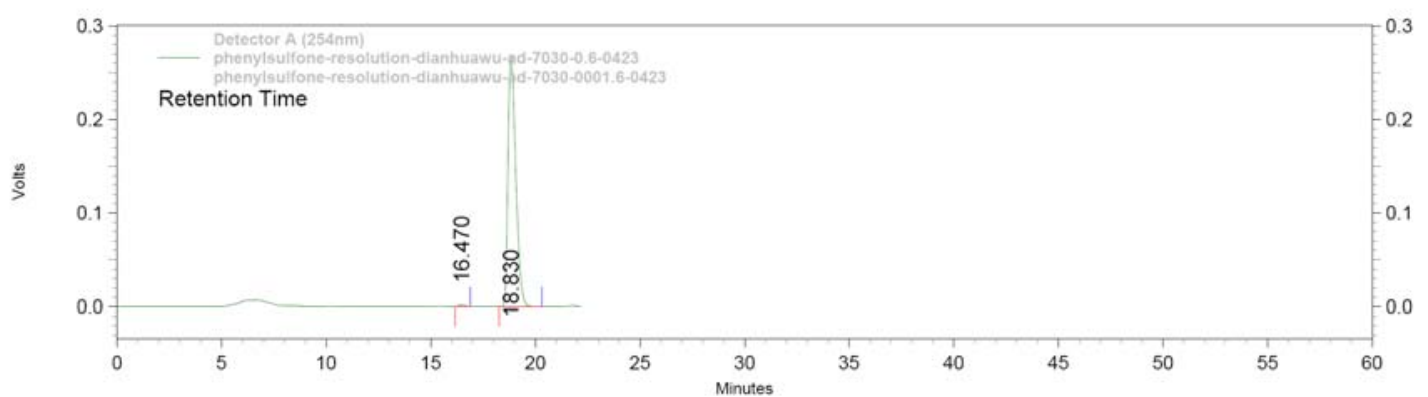

Detector A (254nm)

\begin{tabular}{rrrrrr} 
Pk \# & Retention Time & Area & Area \% & Height & Height \% \\
\hline 1 & 16.470 & 31398 & 0.453 & 1542 & 0.573 \\
2 & 18.830 & 6903579 & 99.547 & 267363 & 99.427
\end{tabular}


Table 4 entry 5

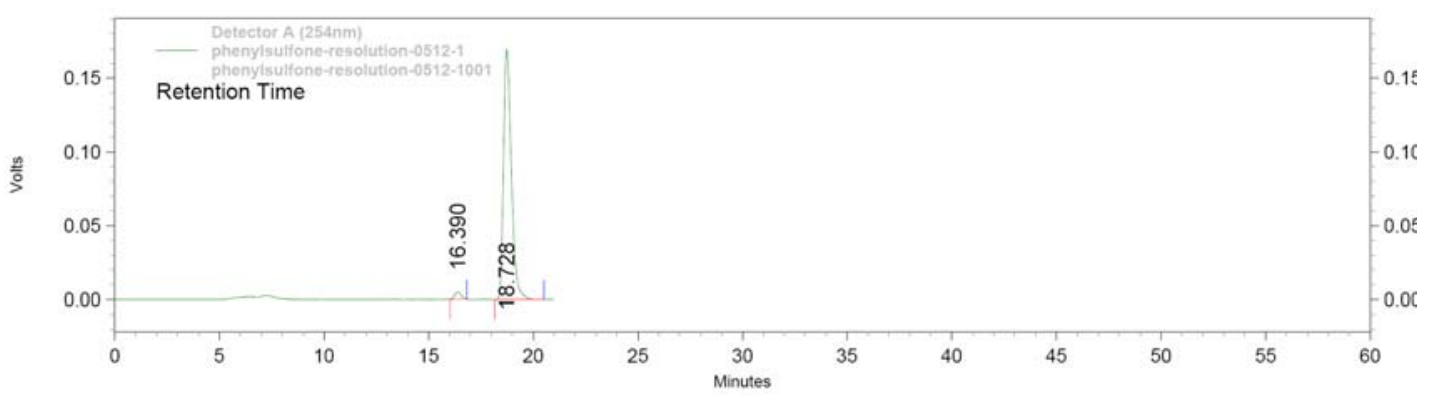

Detector A (254nm)

\begin{tabular}{rrrrrr} 
Pk\# & Retention Time & Area & Area \% & Height & Height \% \\
\hline 1 & 16.390 & 107349 & 2.365 & 5020 & 2.871 \\
2 & 18.728 & 4431435 & 97.635 & 169815 & 97.129
\end{tabular}

\section{Table 4 entry 6}

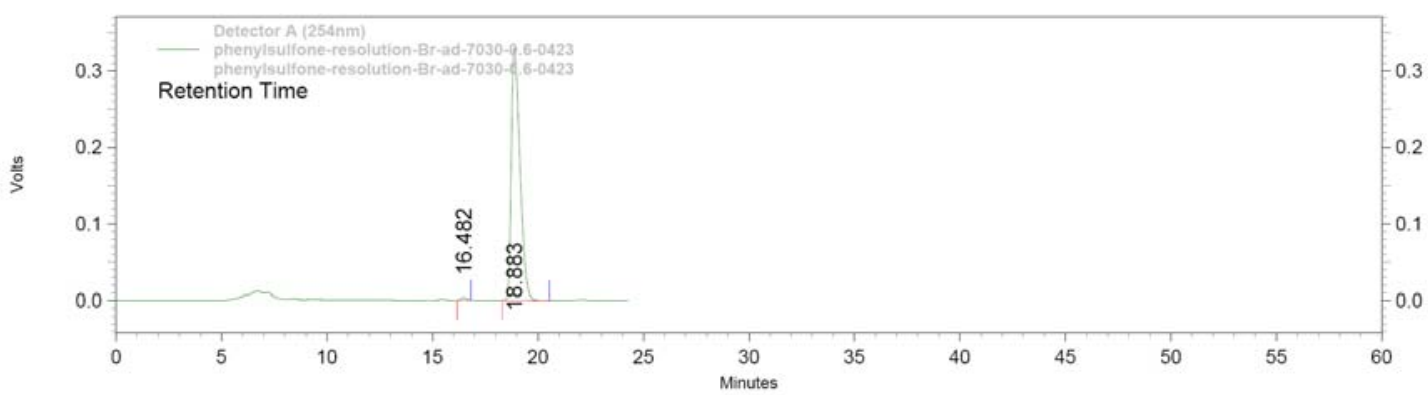

Detector A (254nm)

\begin{tabular}{rrrrrr} 
Pk \# & Retention Time & Area & Area \% & Height & Height \% \\
\hline 1 & 16.482 & 62935 & 0.670 & 3244 & 0.976 \\
2 & 18.883 & 9329727 & 99.330 & 329150 & 99.024
\end{tabular}




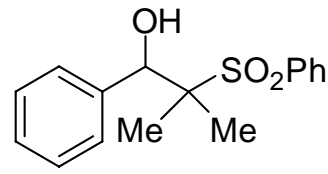

\section{$5 c$ racemate}

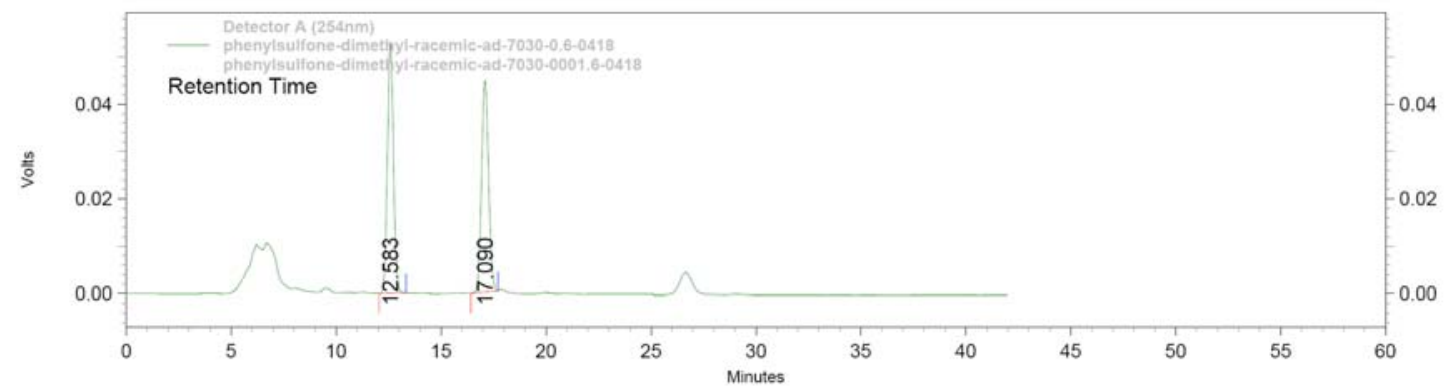

\begin{tabular}{|c|c|c|c|c|c|}
\hline \multicolumn{6}{|c|}{ Detector A (254nm) } \\
\hline Pk\# & Retention Time & Area & Area \% & Height & Height $\%$ \\
\hline 1 & 12.583 & 1051118 & 50.218 & 52671 & 54.084 \\
\hline 2 & 17.090 & 1041988 & 49.782 & 44716 & 45.916 \\
\hline
\end{tabular}

\section{Table 4 entry 7}

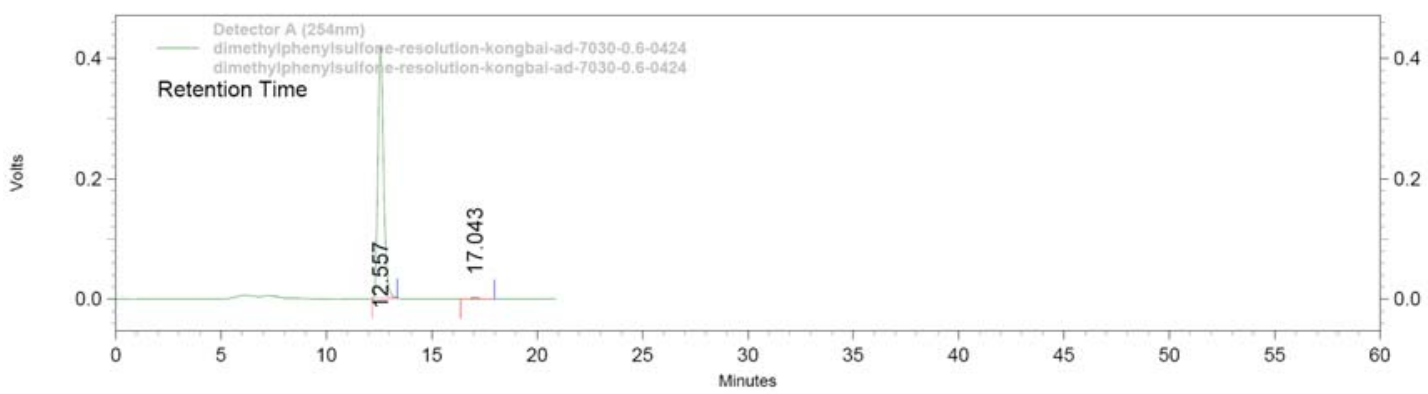

Detector A (254nm)

\begin{tabular}{rrrrrr} 
Pk \# & Retention Time & Area & Area \% & Height & Height \% \\
\hline 1 & 12.557 & 7849671 & 99.100 & 418320 & 99.269 \\
2 & 17.043 & 71301 & 0.900 & 3079 & 0.731
\end{tabular}




\section{Table 4 entry 8}

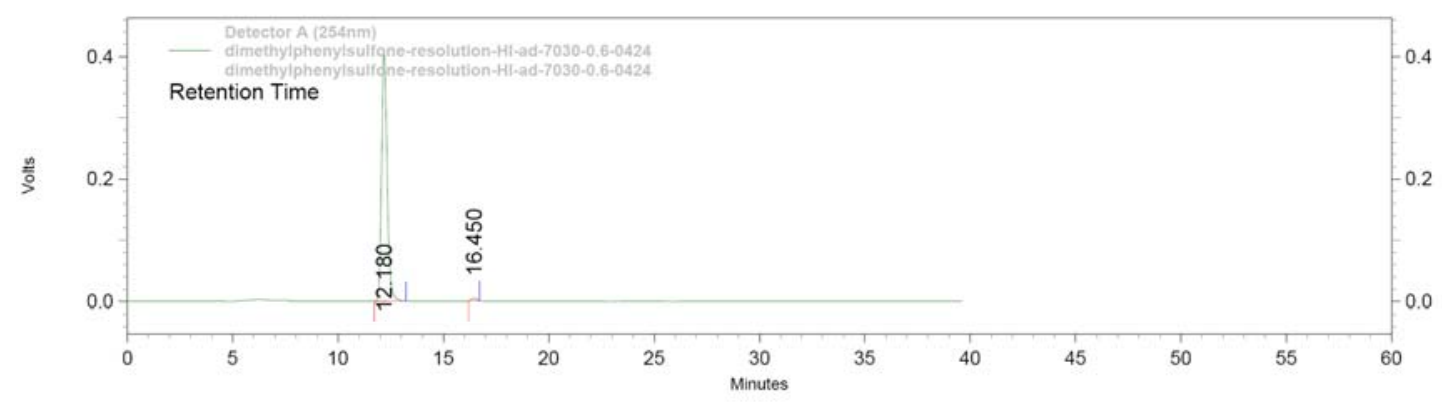

Detector A (254nm)

\begin{tabular}{|c|c|c|c|c|c|}
\hline Pk\# & Retention Time & Area & Area \% & Height & Height $\%$ \\
\hline 1 & 12.180 & 7444986 & 99.024 & 411190 & 98.983 \\
\hline 2 & 16.450 & 73372 & 0.976 & 4224 & 1.017 \\
\hline
\end{tabular}

\section{Table 4 entry 9}

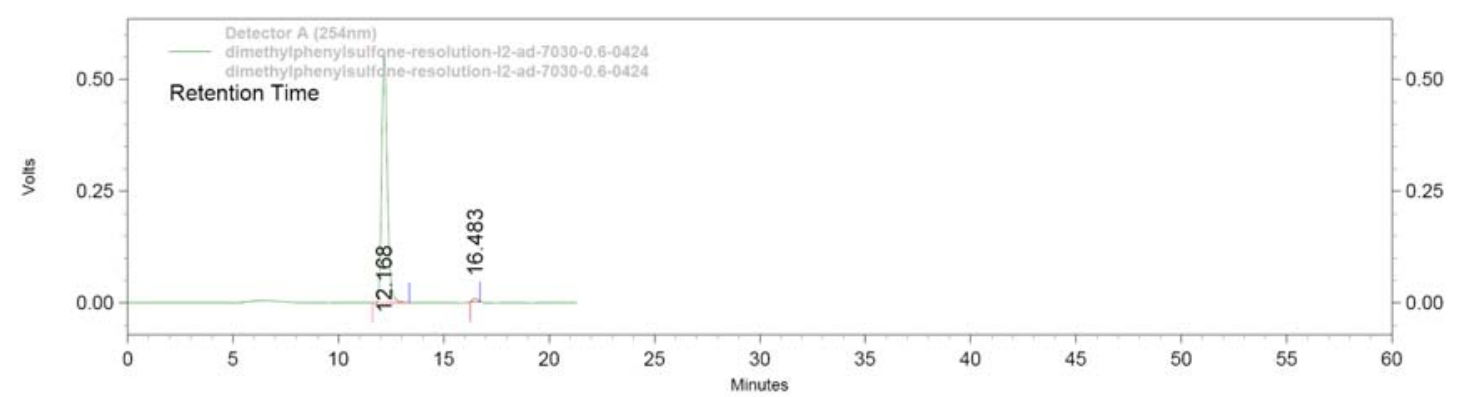

Detector A (254nm)

\begin{tabular}{rrrrrr} 
Pk\# & Retention Time & Area & Area \% & Height & Height \% \\
\hline 1 & 12.168 & 10252629 & 98.830 & 564347 & 98.693 \\
2 & 16.483 & 121366 & 1.170 & 7476 & 1.307
\end{tabular}




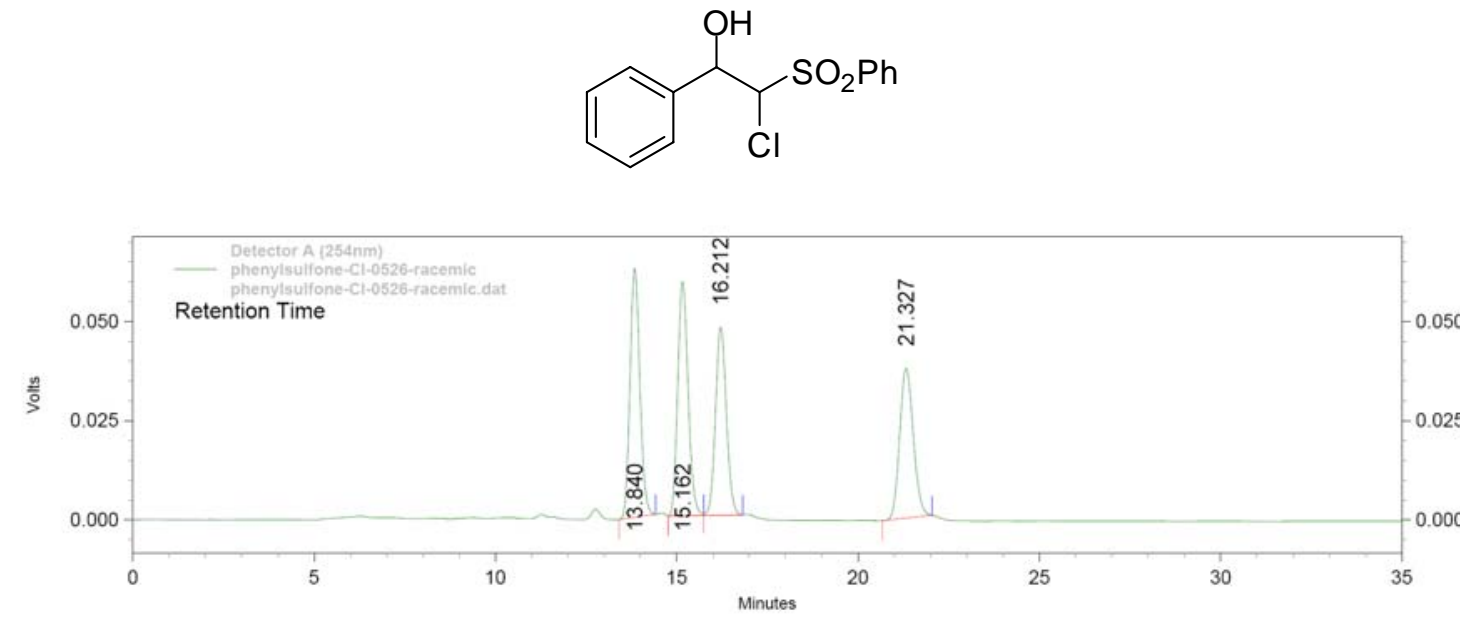

Detector A (254nm)

Pk\# Retention Time

Area

Area \%

Height

Height \%

$\begin{array}{lrrrrr}1 & 13.840 & 1183969 & 27.356 & 62747 & 30.354 \\ 2 & 15.162 & 1169533 & 27.022 & 58852 & 28.469 \\ 3 & 16.212 & 989064 & 22.853 & 47365 & 22.913 \\ 4 & 21.327 & 985436 & 22.769 & 37756 & 18.264\end{array}$

Table 4 entry 10

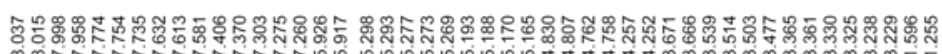

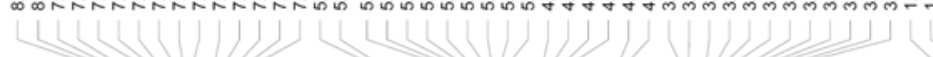

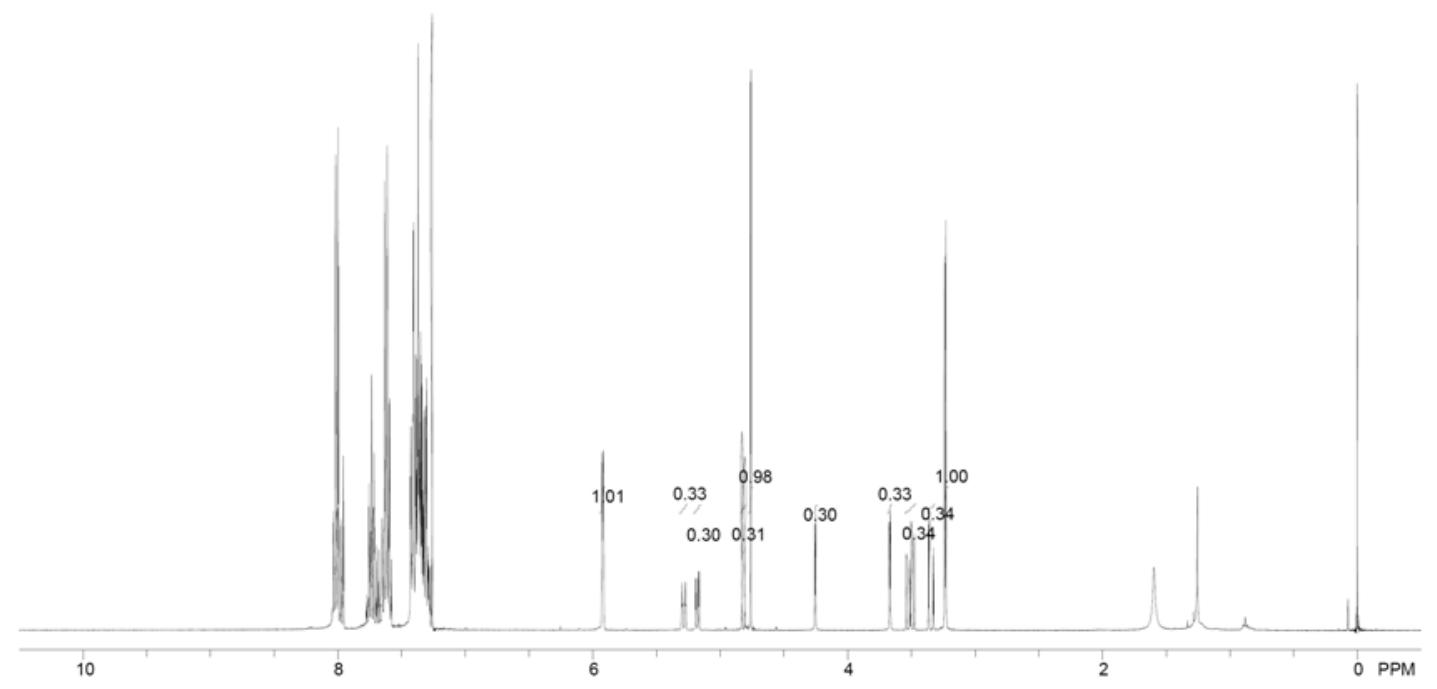



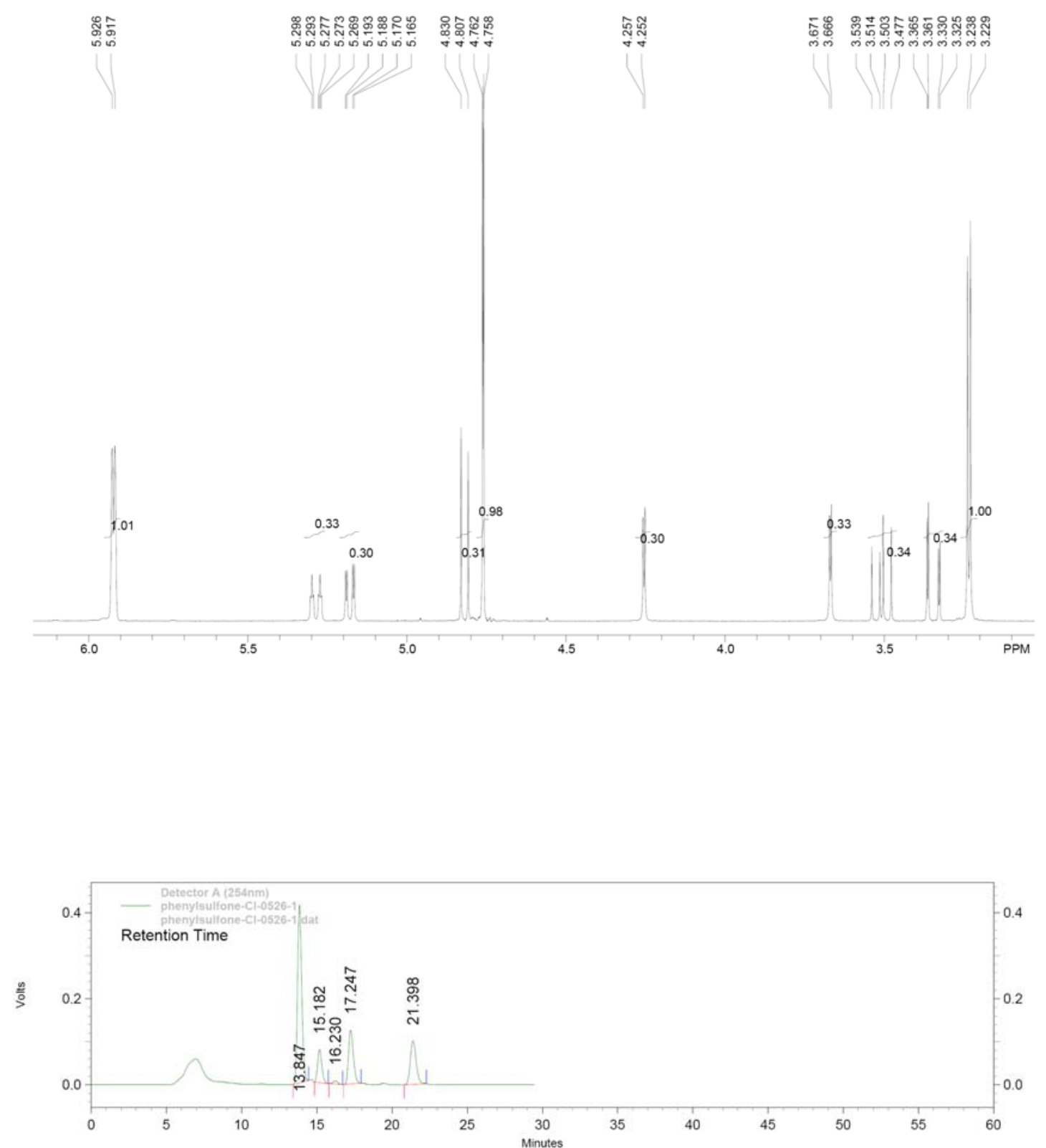

Detector A (254nm)

Pk\# Retention Time

\begin{tabular}{|c|c|c|c|c|c|}
\hline 1 & 13.847 & 8009370 & 52.823 & 412984 & 57.117 \\
\hline 2 & 15.182 & 1503173 & 9.914 & 76903 & 10.636 \\
\hline 3 & 16.230 & 153956 & 1.015 & 7232 & 1.000 \\
\hline 4 & 17.247 & 2782866 & 18.353 & 124880 & 17.271 \\
\hline 5 & 21.398 & 2713385 & 17.895 & 101053 & 13.976 \\
\hline
\end{tabular}

9.914

1.015

18.353

17.895

Height

Height \% 


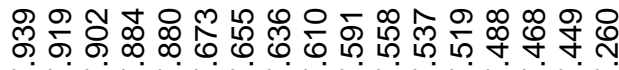

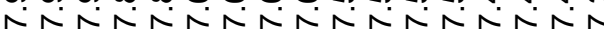

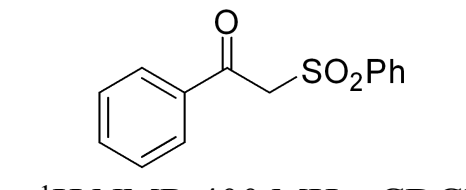

1a ${ }^{1} \mathrm{H}$ NMR $400 \mathrm{MHz}, \mathrm{CDCl}_{3}$ 


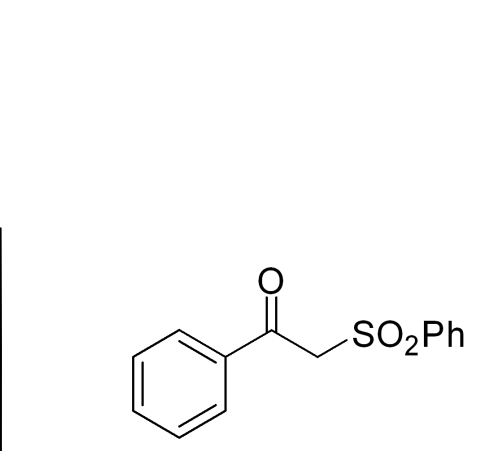

1a ${ }^{13} \mathrm{C} \mathrm{NMR} 100 \mathrm{MHz}, \mathrm{CDCl}_{3}$ 


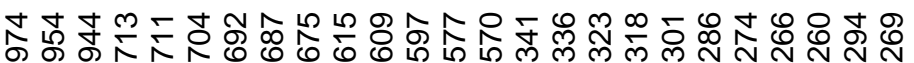

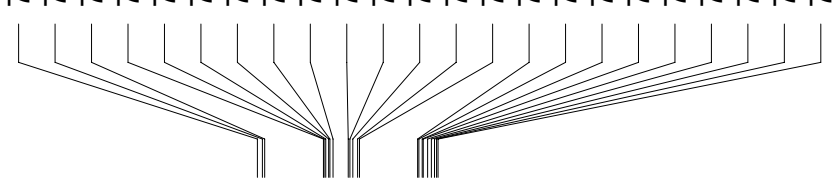

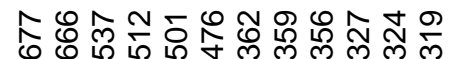

लंmलंलmलm

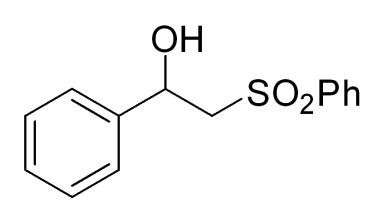

2a ${ }^{1} \mathrm{H}$ NMR $400 \mathrm{MHz}, \mathrm{CDCl}_{3}$ 


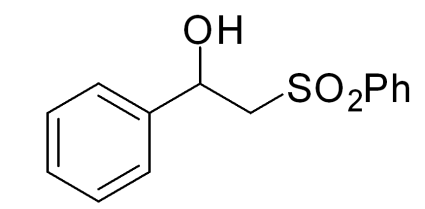

2a ${ }^{13} \mathrm{C}$ NMR $100 \mathrm{MHz}, \mathrm{CDCl}_{3}$ 


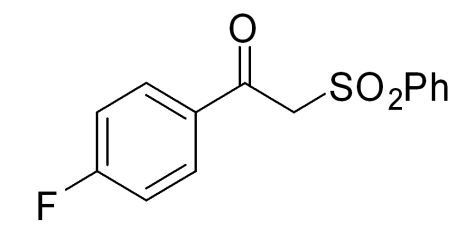

1b ${ }^{1} \mathrm{H}$ NMR $400 \mathrm{MHz}, \mathrm{CDCl}_{3}$

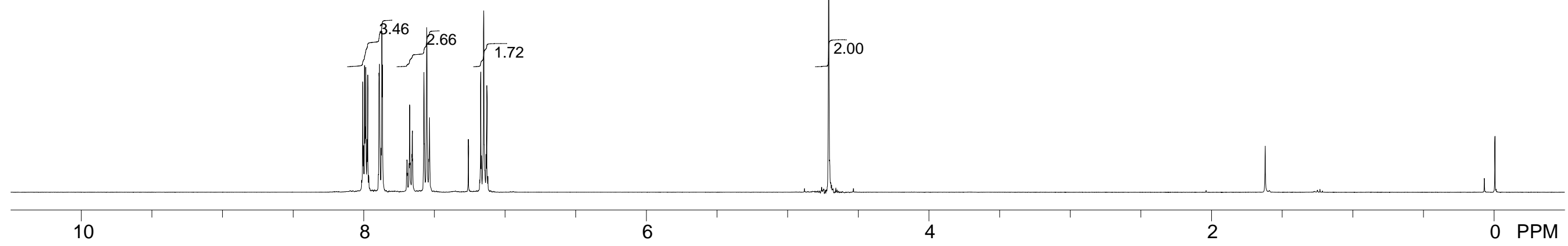




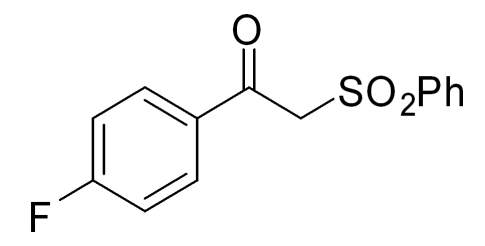

1b ${ }^{13} \mathrm{C}$ NMR $100 \mathrm{MHz}, \mathrm{CDCl}_{3}$ 


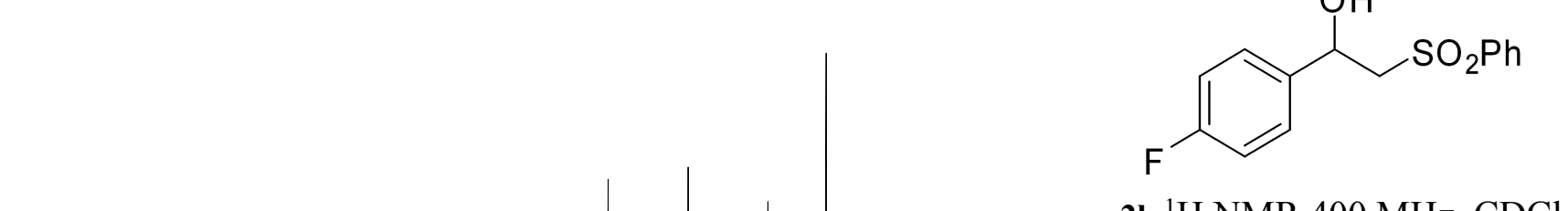

2b ${ }^{1} \mathrm{H}$ NMR $400 \mathrm{MHz}, \mathrm{CDCl}_{3}$ 
(

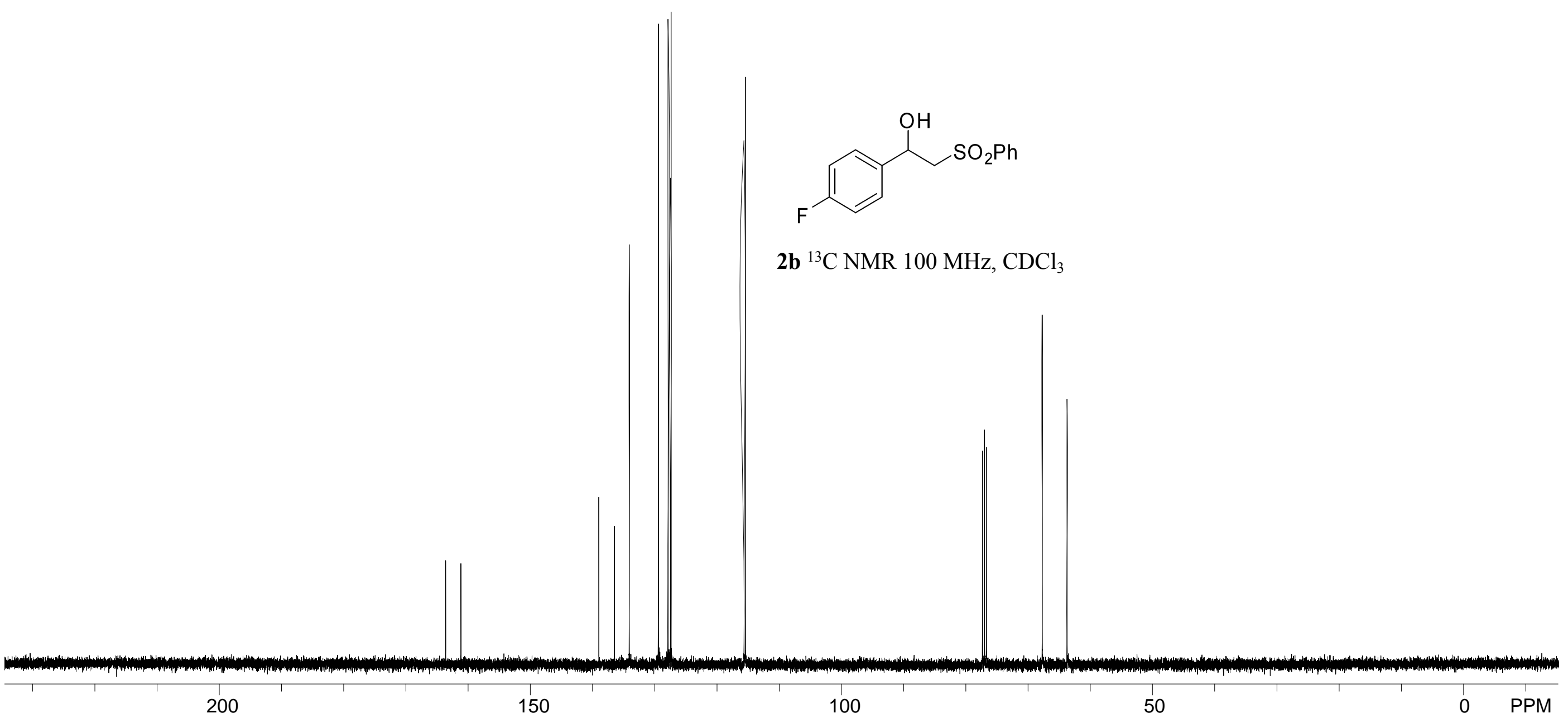




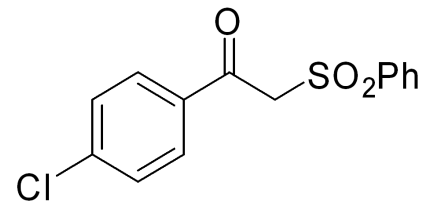

1c ${ }^{1} \mathrm{H}$ NMR $400 \mathrm{MHz}, \mathrm{CDCl}_{3}$ 


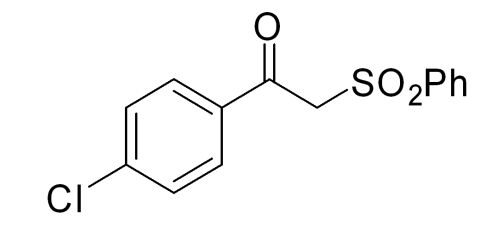

1c ${ }^{13} \mathrm{C} \mathrm{NMR} 100 \mathrm{MHz}, \mathrm{CDCl}_{3}$ 


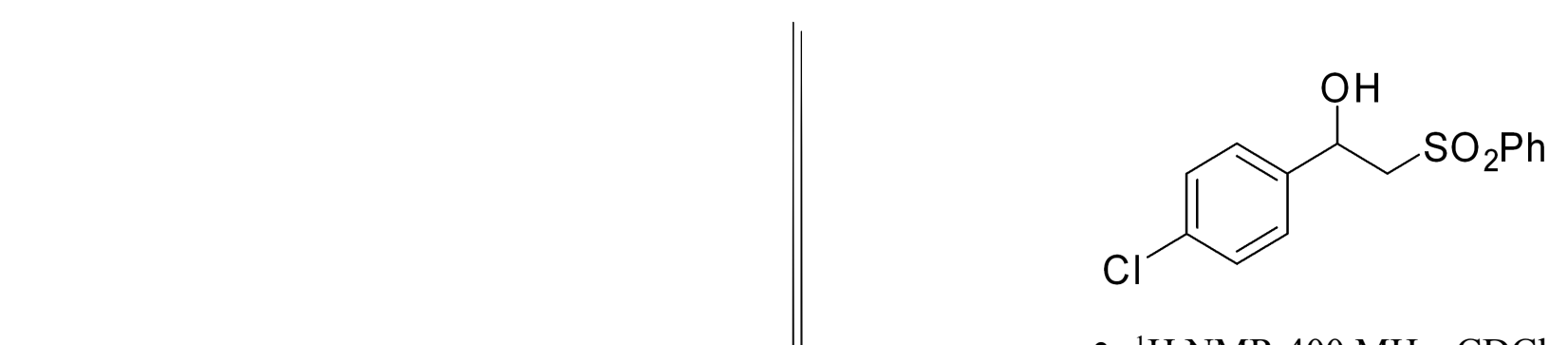

2c ${ }^{1} \mathrm{H}$ NMR $400 \mathrm{MHz}, \mathrm{CDCl}_{3}$ 


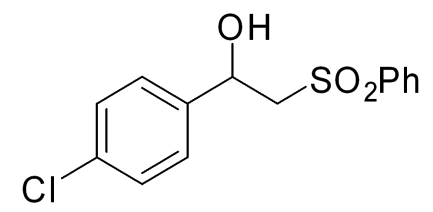

2c ${ }^{13} \mathrm{C}$ NMR $100 \mathrm{MHz}, \mathrm{CDCl}_{3}$ 


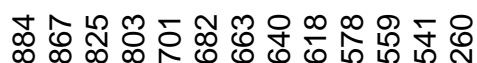

ํ.

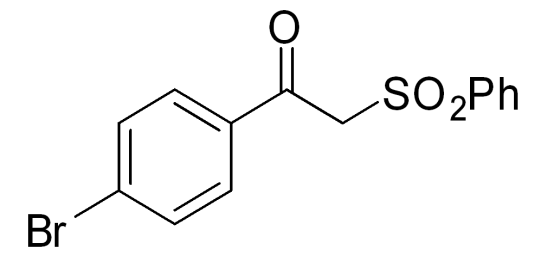

1d ${ }^{1} \mathrm{H}$ NMR $400 \mathrm{MHz}, \mathrm{CDCl}_{3}$ 


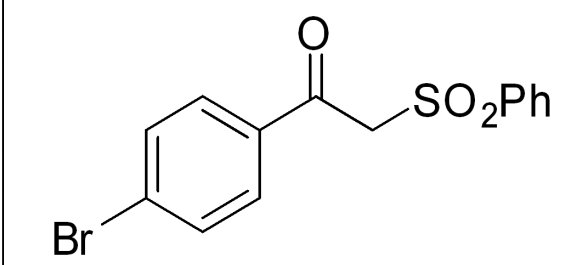

1d ${ }^{13} \mathrm{C}$ NMR $100 \mathrm{MHz}, \mathrm{CDCl}_{3}$ 


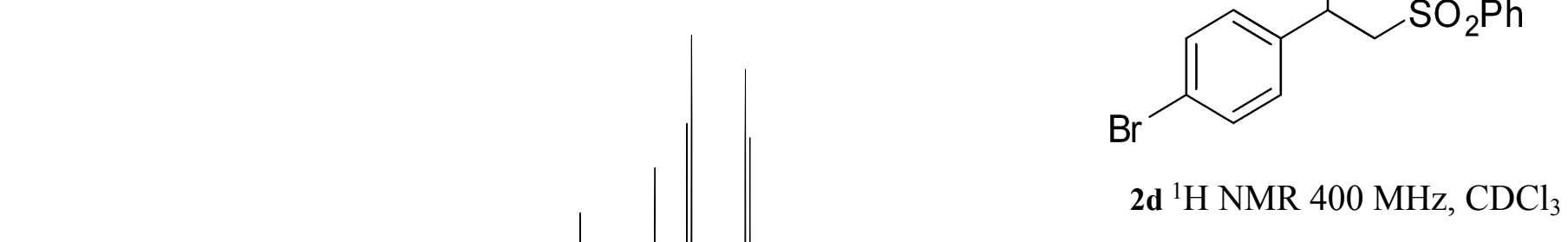



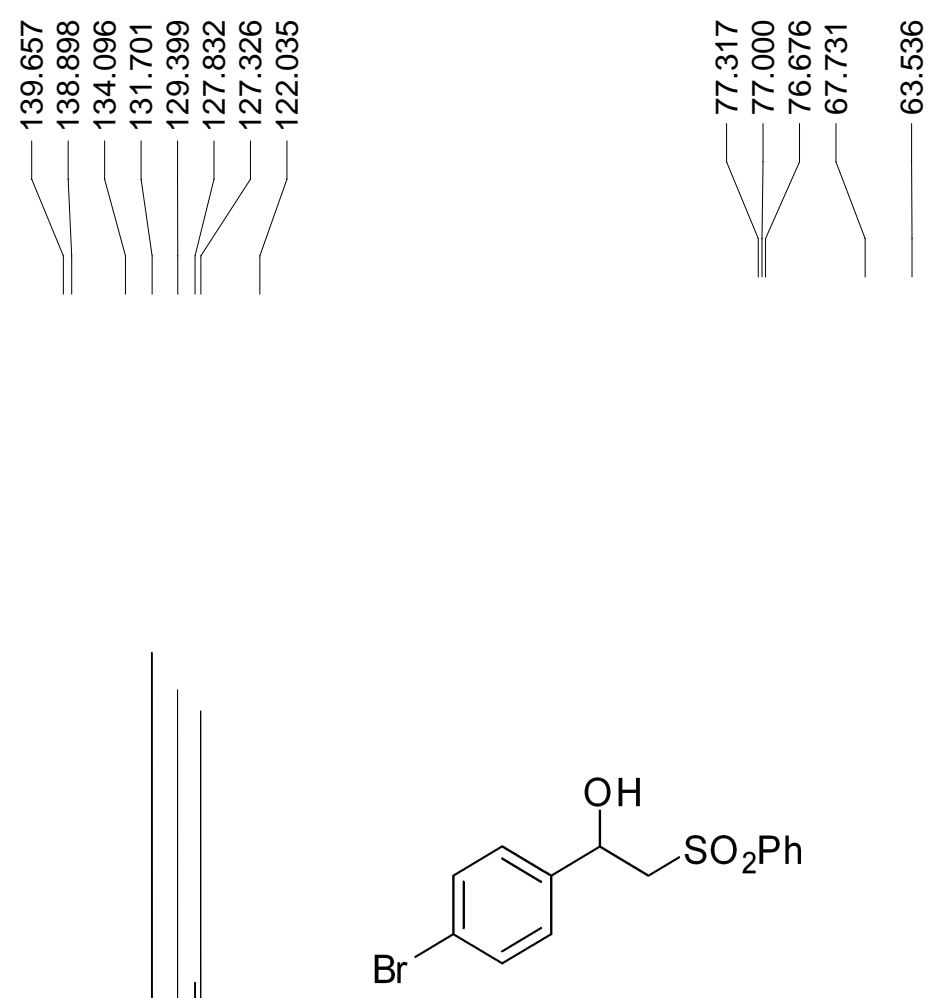

2d ${ }^{13} \mathrm{C}$ NMR $100 \mathrm{MHz}, \mathrm{CDCl}_{3}$ 

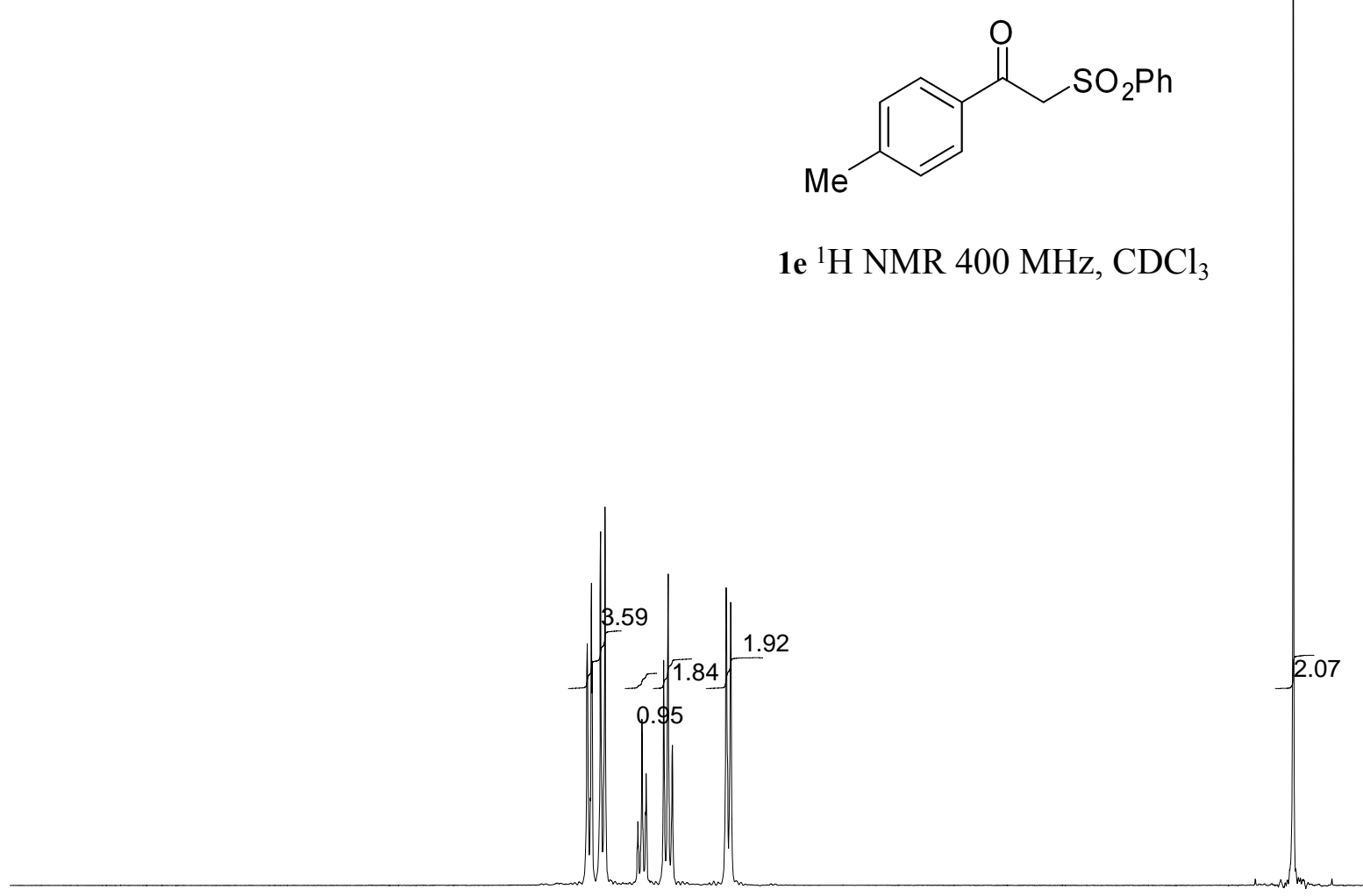

1e ${ }^{1} \mathrm{H}$ NMR $400 \mathrm{MHz}, \mathrm{CDCl}_{3}$ 


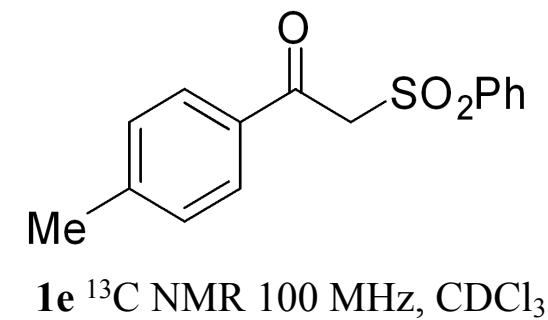




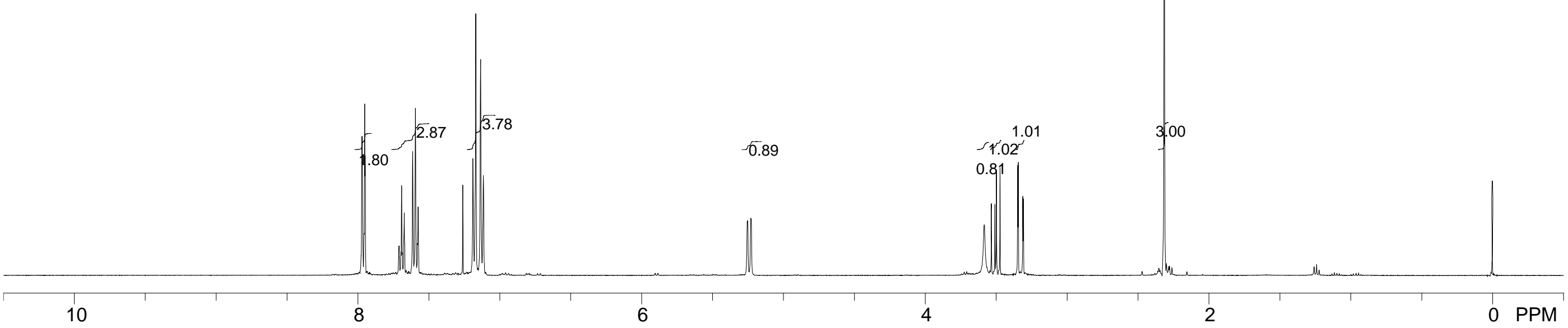




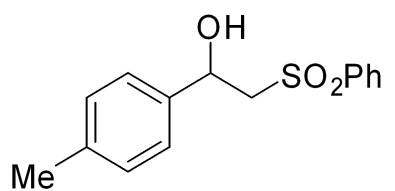

2e ${ }^{13} \mathrm{C} \mathrm{NMR} 100 \mathrm{MHz}, \mathrm{CDCl}_{3}$ 


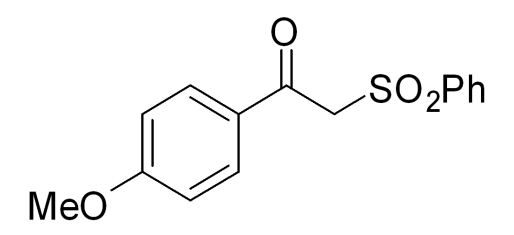

1f ${ }^{1} \mathrm{H}$ NMR $400 \mathrm{MHz}, \mathrm{CDCl}_{3}$ 

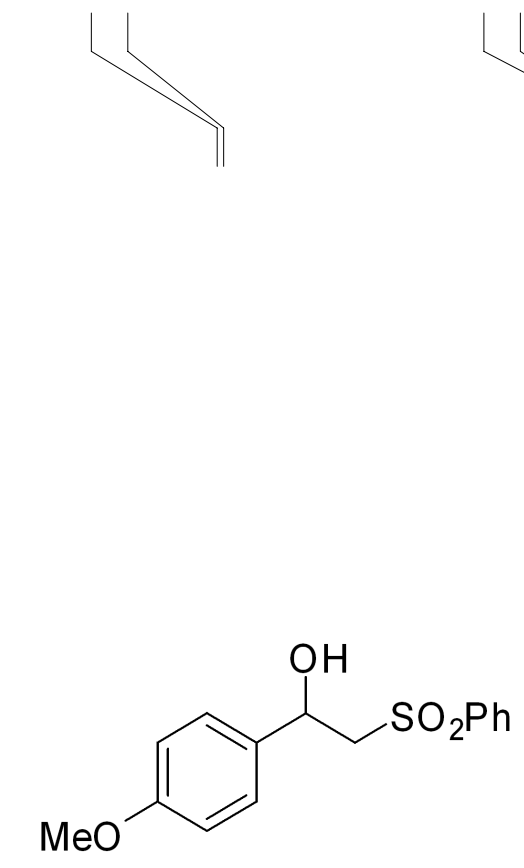

2f ${ }^{1} \mathrm{H}$ NMR $400 \mathrm{MHz}, \mathrm{CDCl}_{3}$
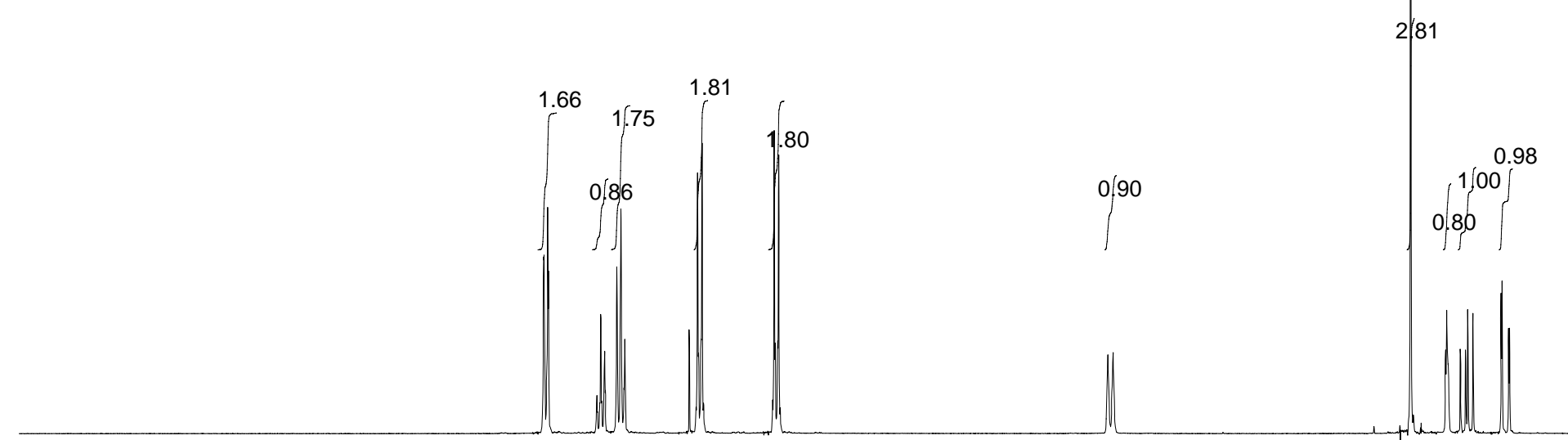


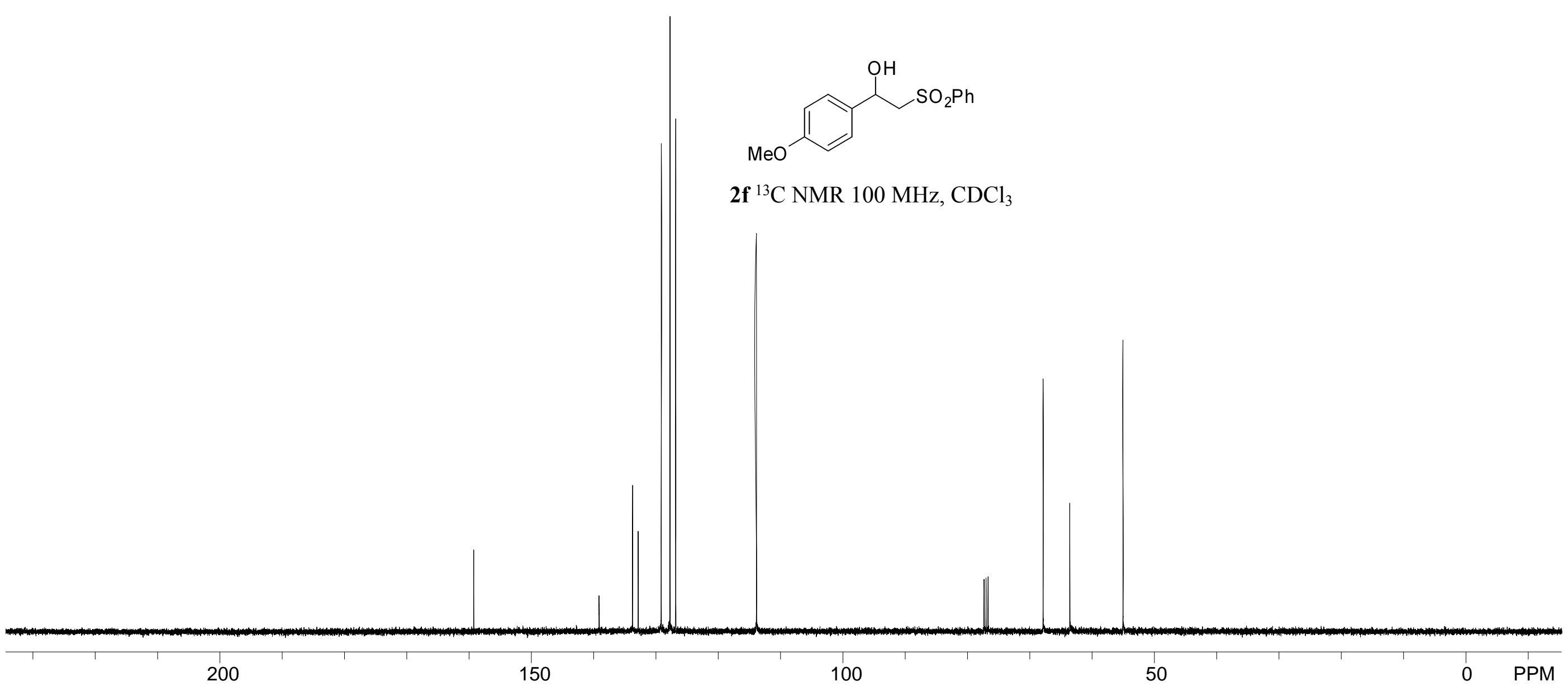




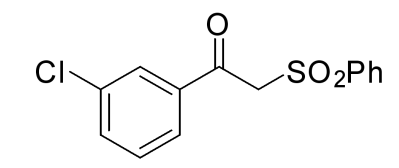

1g ${ }^{1} \mathrm{H}$ NMR $400 \mathrm{MHz}, \mathrm{CDCl}_{3}$ 


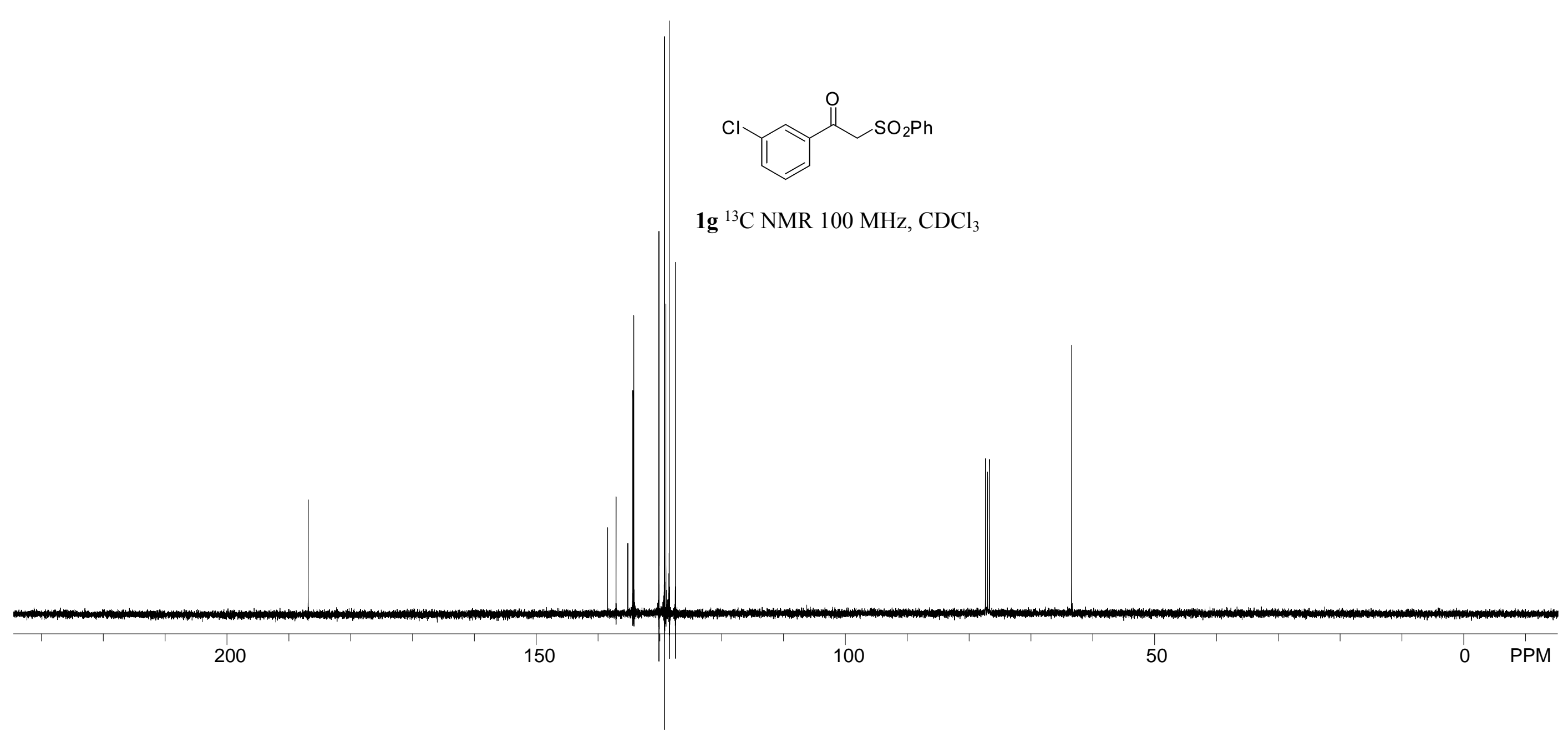



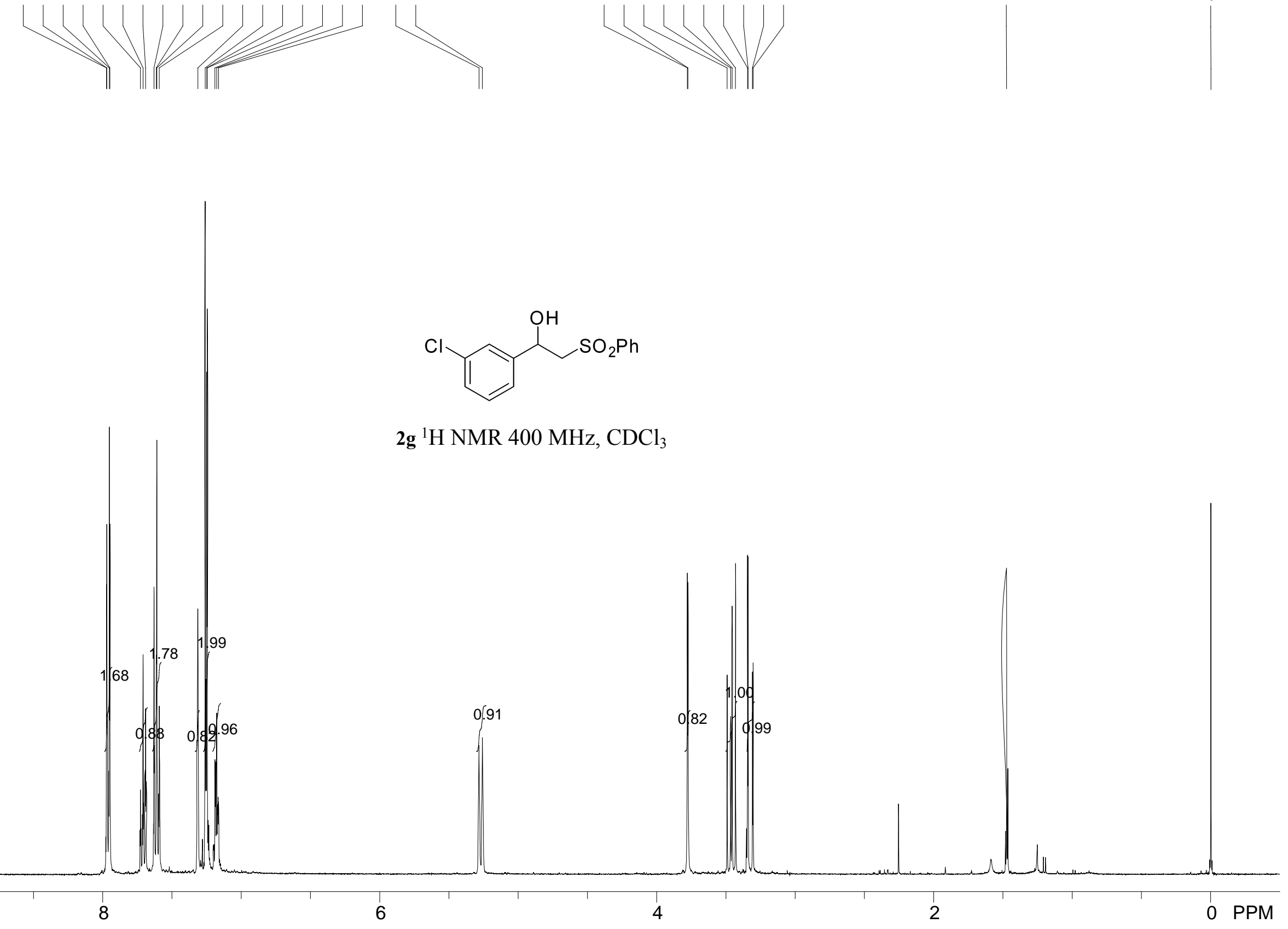


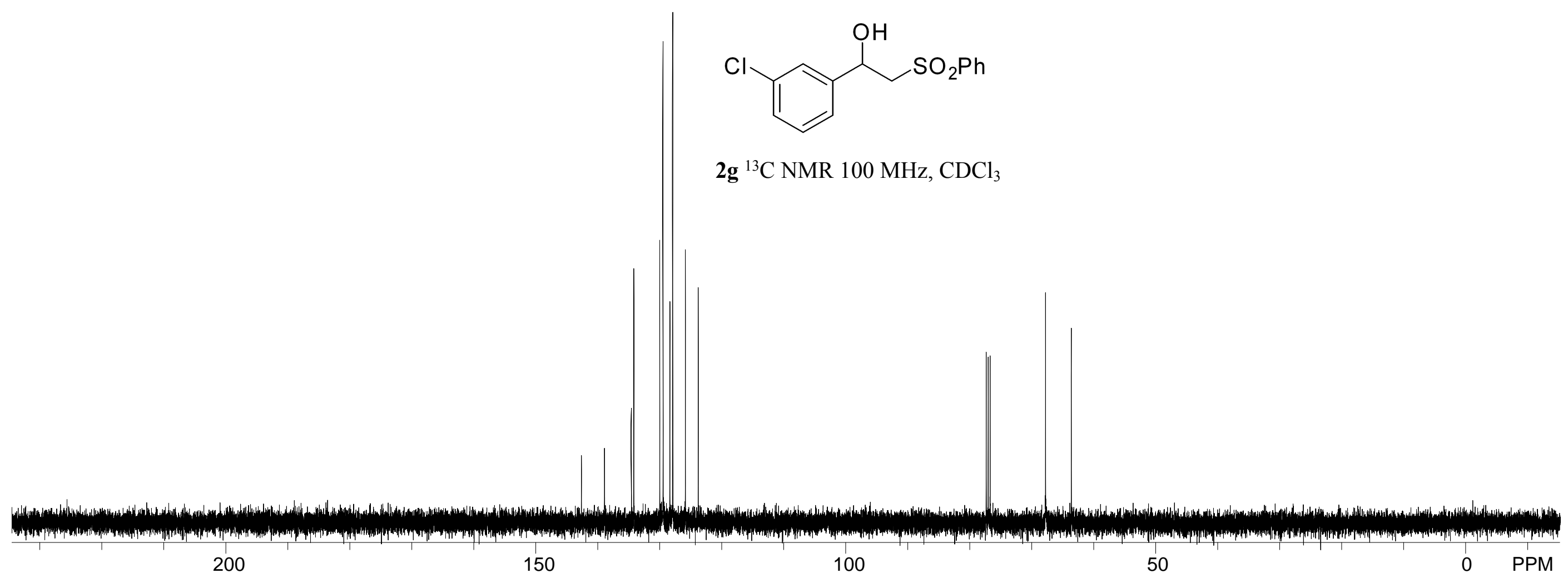




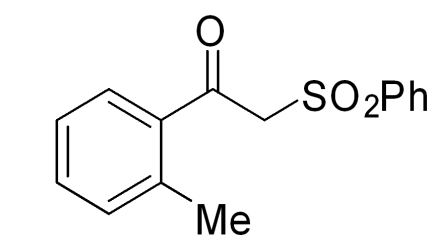

1h ${ }^{1} \mathrm{H}$ NMR $400 \mathrm{MHz}, \mathrm{CDCl}_{3}$ 


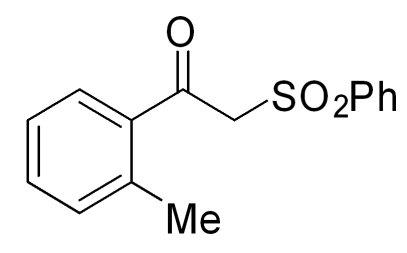

1h ${ }^{1} \mathrm{H}$ NMR $400 \mathrm{MHz}, \mathrm{CDCl}_{3}$ 

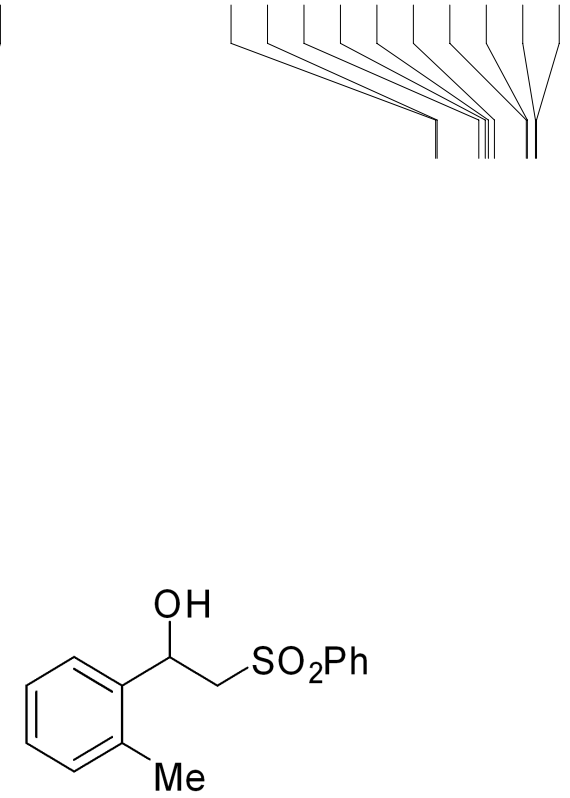

2h ${ }^{1} \mathrm{H}$ NMR $400 \mathrm{MHz}, \mathrm{CDCl}_{3}$ 


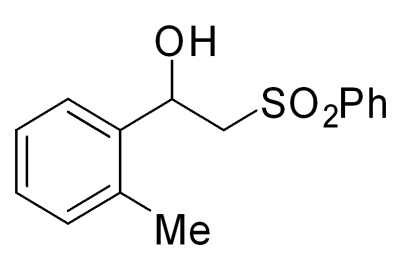

2h ${ }^{13} \mathrm{C}$ NMR $100 \mathrm{MHz}, \mathrm{CDCl}_{3}$ 

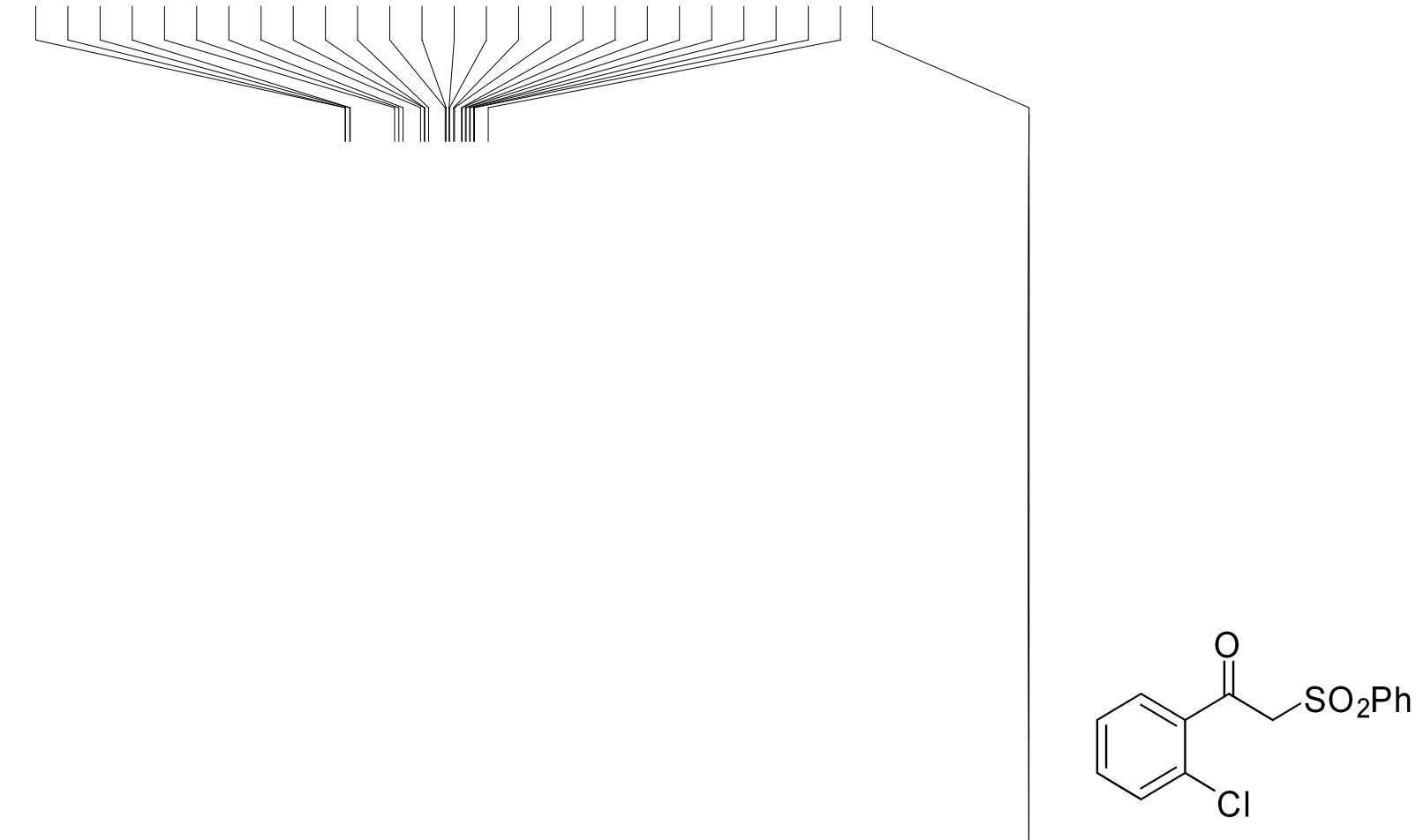

1i ${ }^{1} \mathrm{H}$ NMR $400 \mathrm{MHz}, \mathrm{CDCl}_{3}$

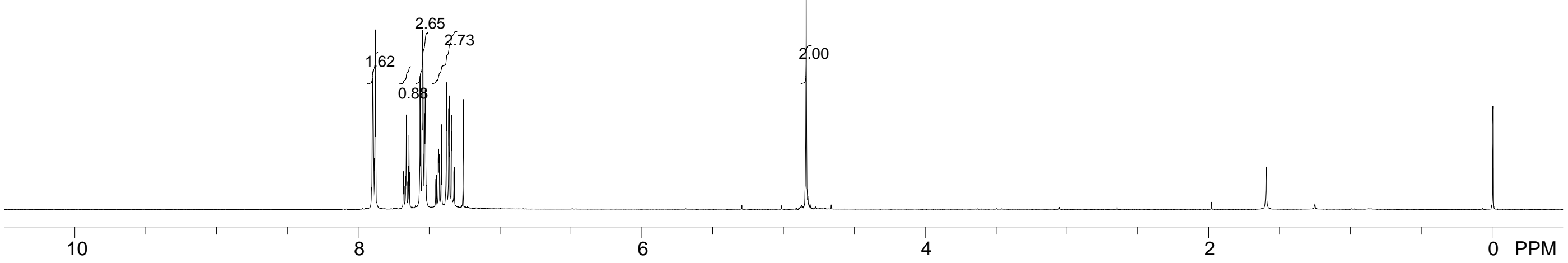




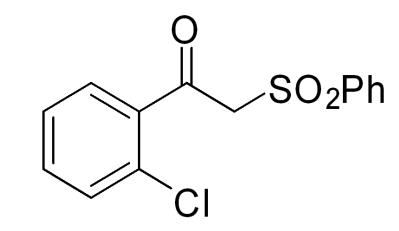

1i ${ }^{13} \mathrm{C}$ NMR $100 \mathrm{MHz}, \mathrm{CDCl}_{3}$ 


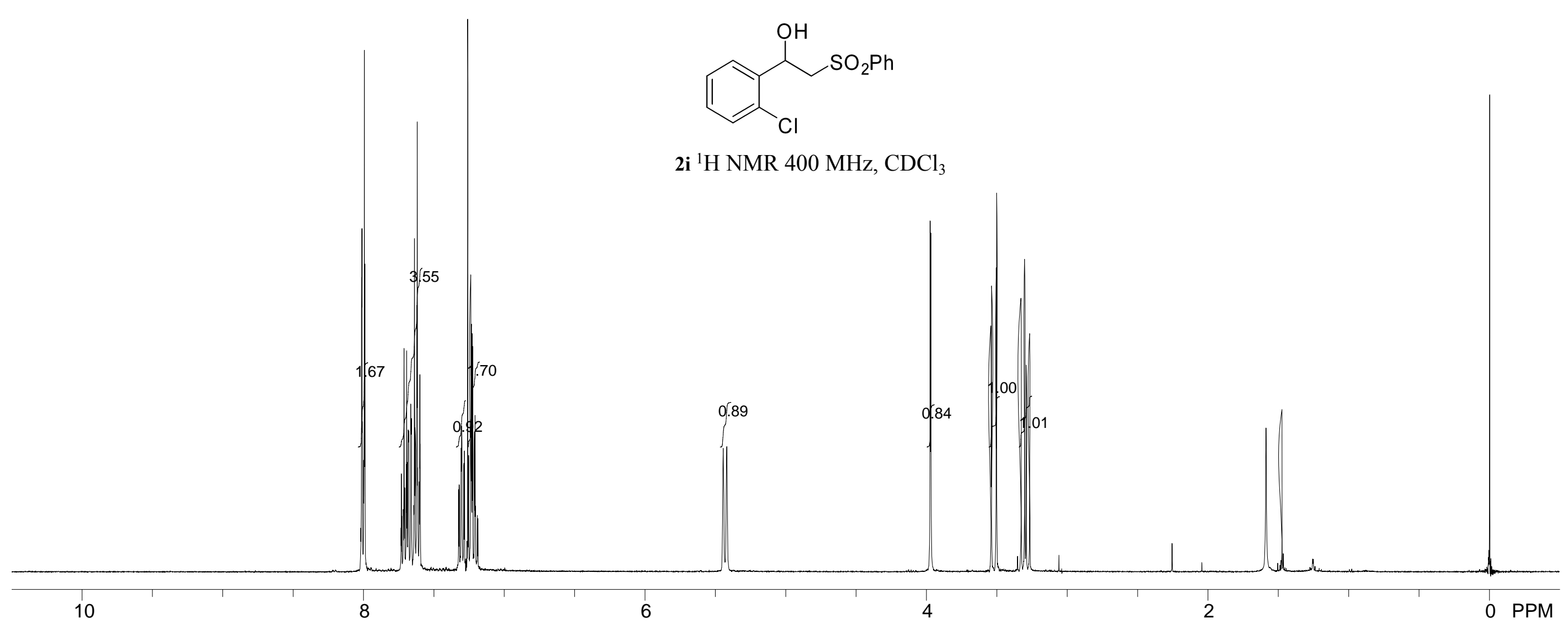



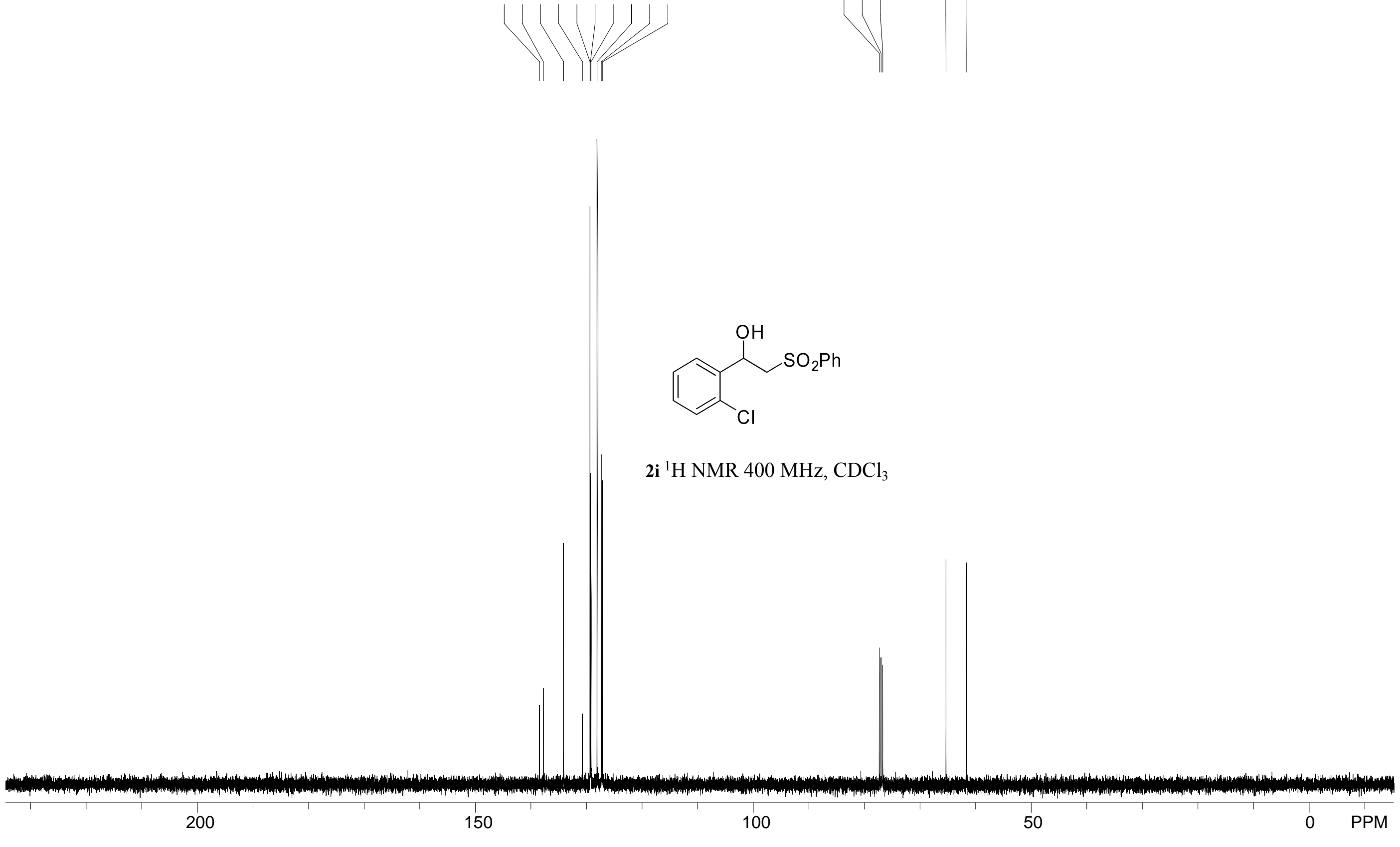


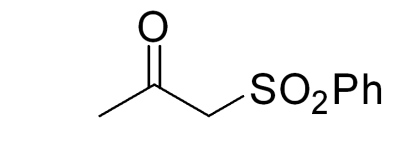

$\mathbf{1 j}^{1} \mathrm{H}$ NMR $400 \mathrm{MHz}, \mathrm{CDCl}_{3}$ 


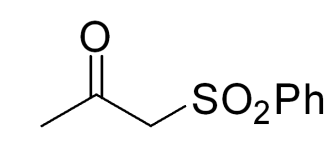

1j ${ }^{13} \mathrm{C}$ NMR $100 \mathrm{MHz}, \mathrm{CDCl}_{3}$ 


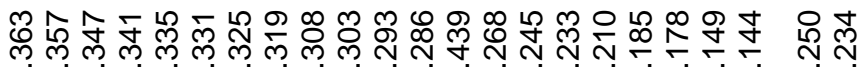

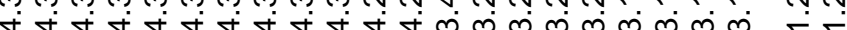
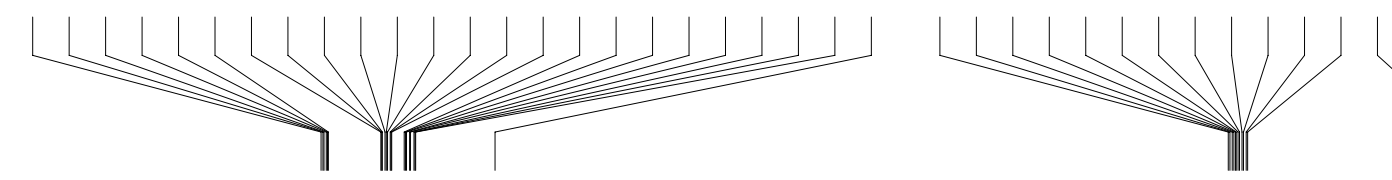

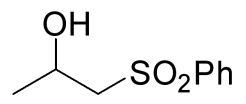

2j ${ }^{1} \mathrm{H}$ NMR $400 \mathrm{MHz}, \mathrm{CDCl}_{3}$ 
离売品

吕

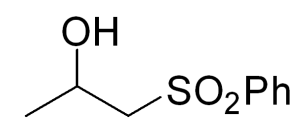

2j ${ }^{13} \mathrm{C}$ NMR $100 \mathrm{MHz}, \mathrm{CDCl}_{3}$ 


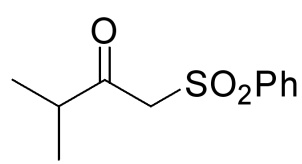

1k ${ }^{1} \mathrm{H}$ NMR $400 \mathrm{MHz}, \mathrm{CDCl}_{3}$ 


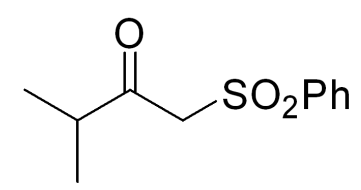

1k ${ }^{13} \mathrm{C}$ NMR $100 \mathrm{MHz}, \mathrm{CDCl}_{3}$

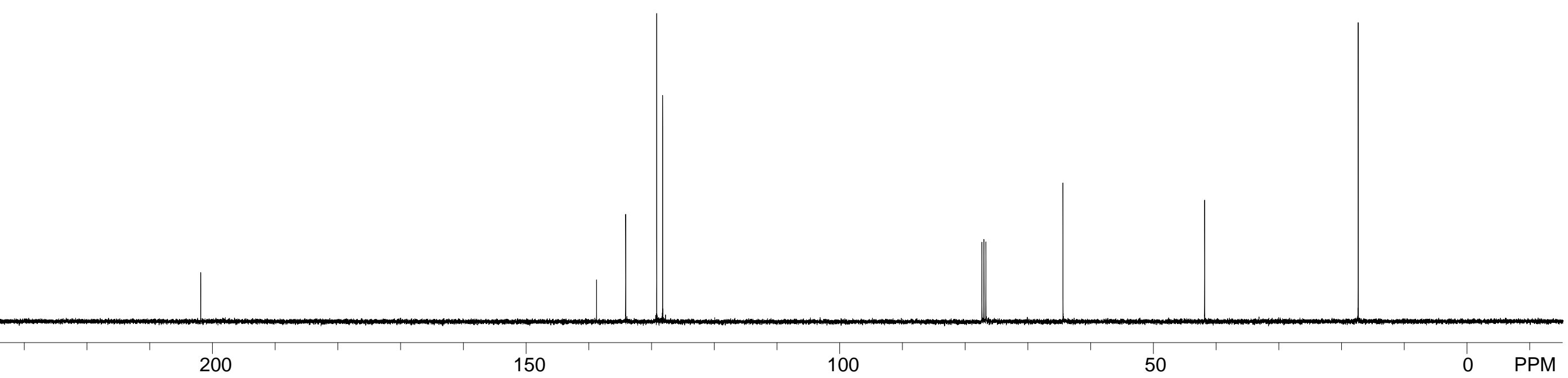



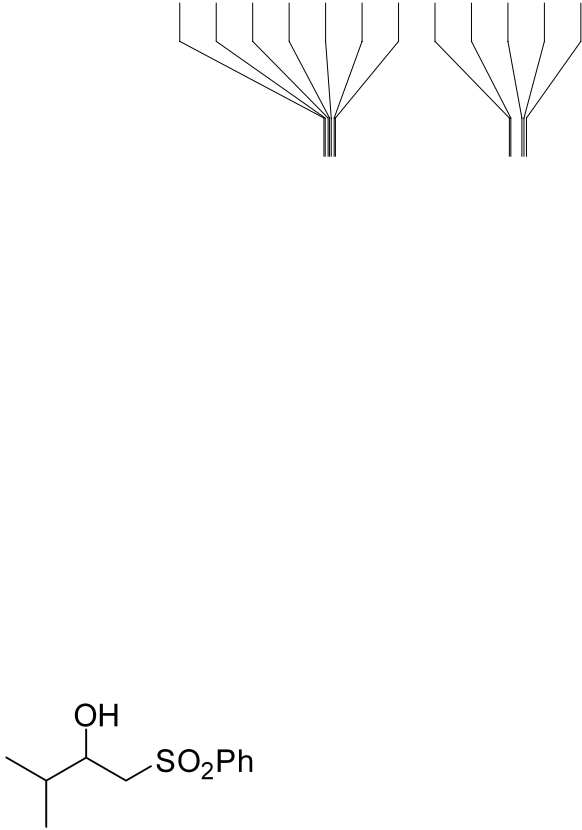

2k ${ }^{1} \mathrm{H}$ NMR $400 \mathrm{MHz}, \mathrm{CDCl}_{3}$

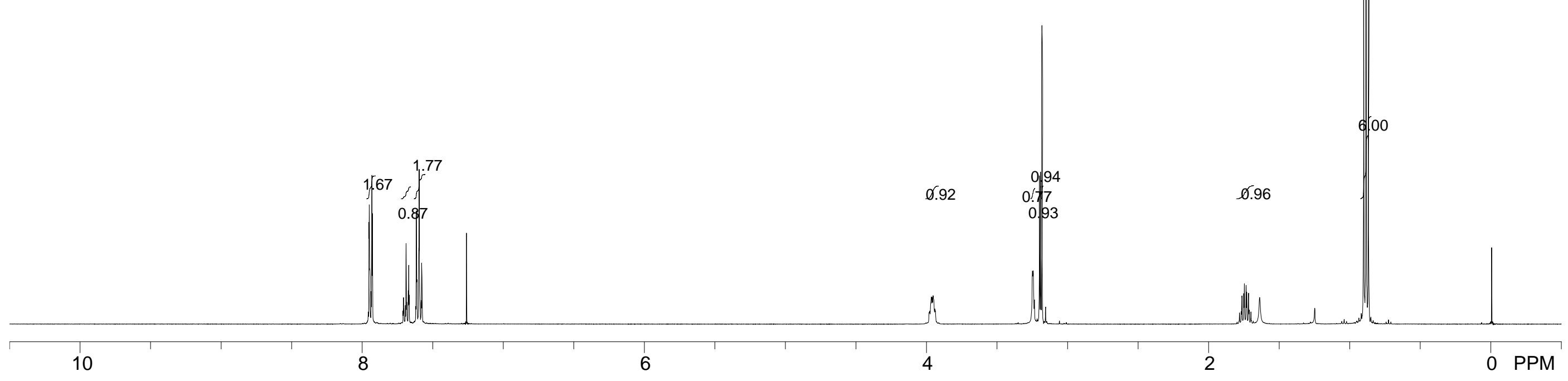




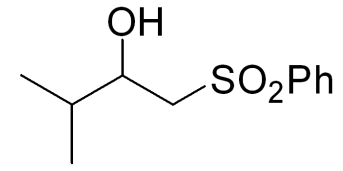

2k ${ }^{13} \mathrm{C}$ NMR $100 \mathrm{MHz}, \mathrm{CDCl}_{3}$ 

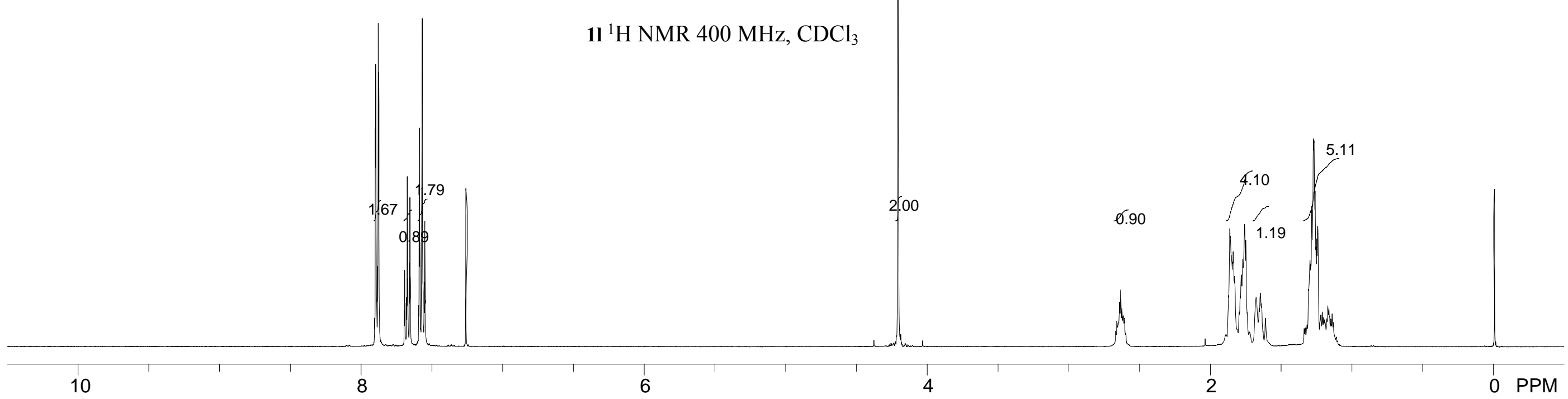


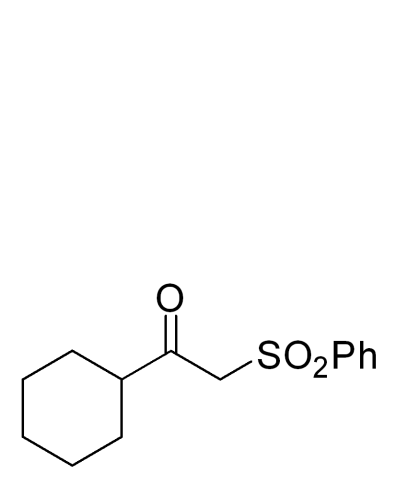

1I ${ }^{13} \mathrm{C}$ NMR $100 \mathrm{MHz}, \mathrm{CDCl}_{3}$ 


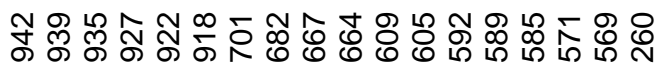
g.$$
1+1+1+1+1
$$

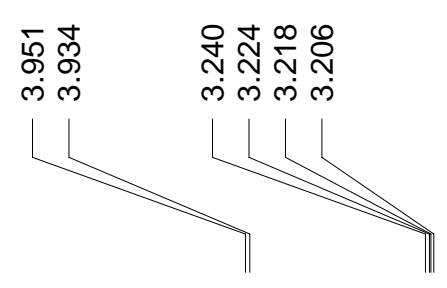

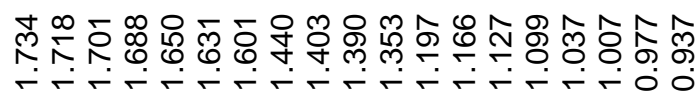

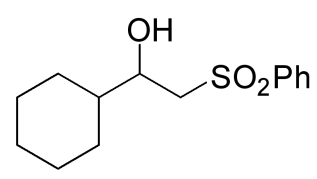

2l ${ }^{1} \mathrm{H}$ NMR $400 \mathrm{MHz}, \mathrm{CDCl}_{3}$ 


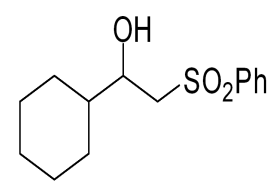

2I ${ }^{13} \mathrm{C}$ NMR $100 \mathrm{MHz}, \mathrm{CDCl}_{3}$ 

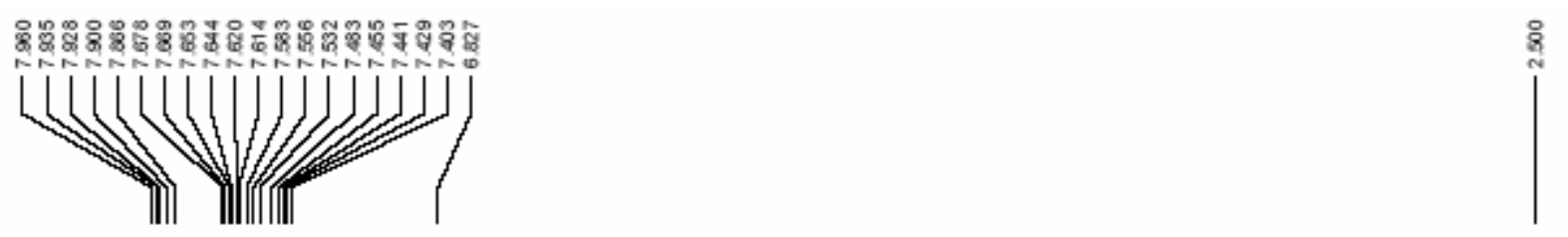<smiles>O=C(c1ccccc1)C(I)S(=O)(=O)c1ccccc1</smiles>

4a ${ }^{1} \mathrm{H}$ NMR 300 MHz, DMSO-D ${ }^{6}$

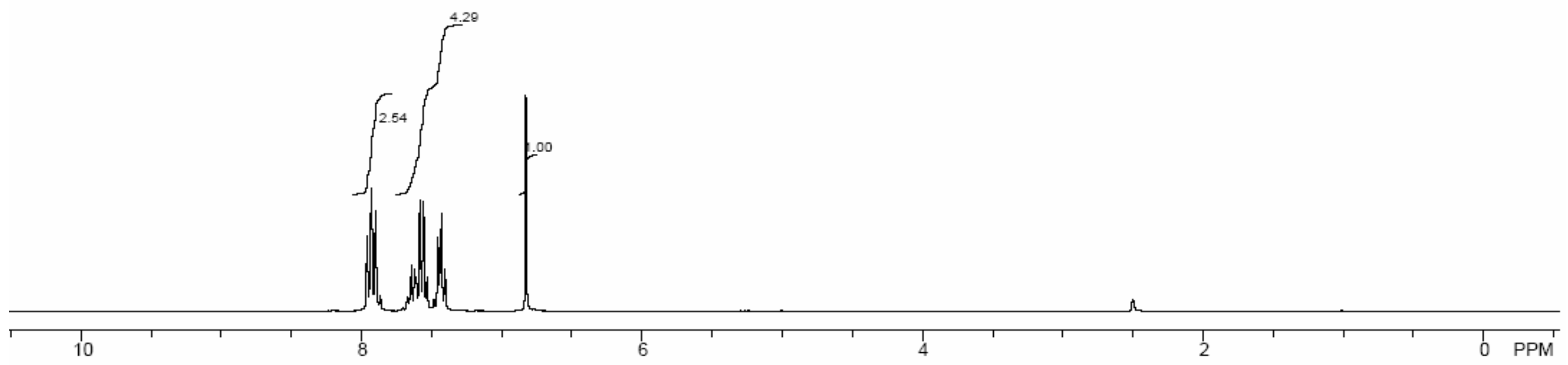




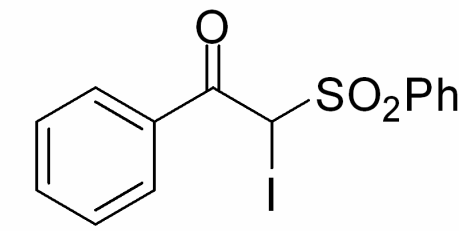

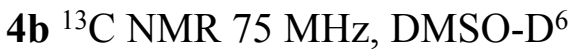

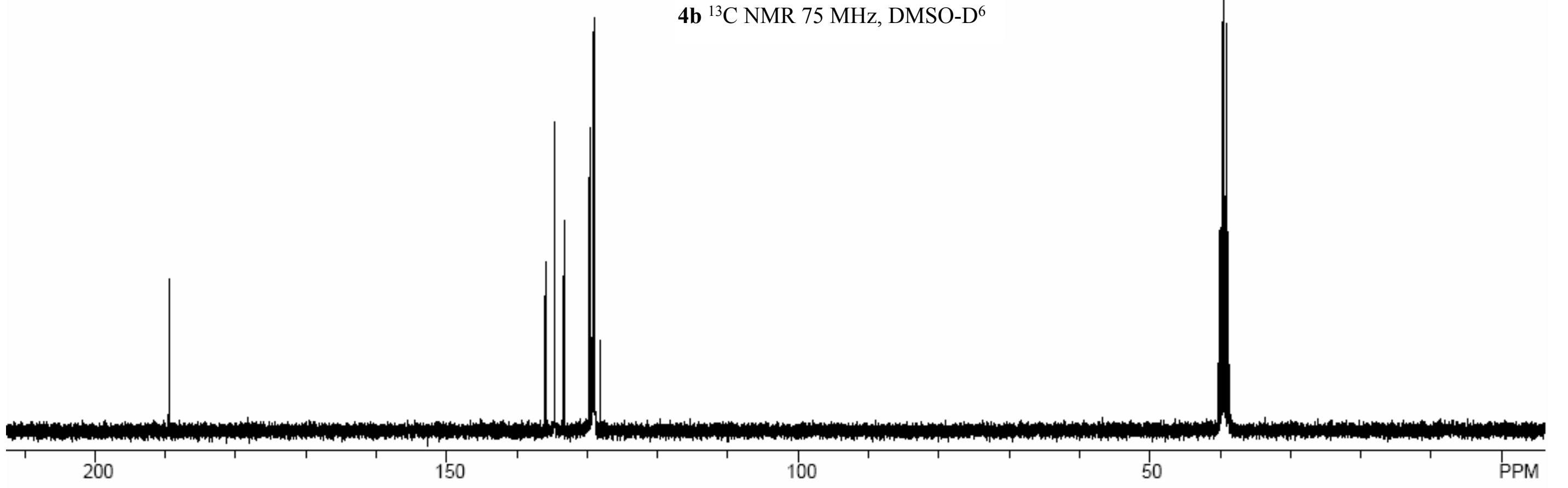



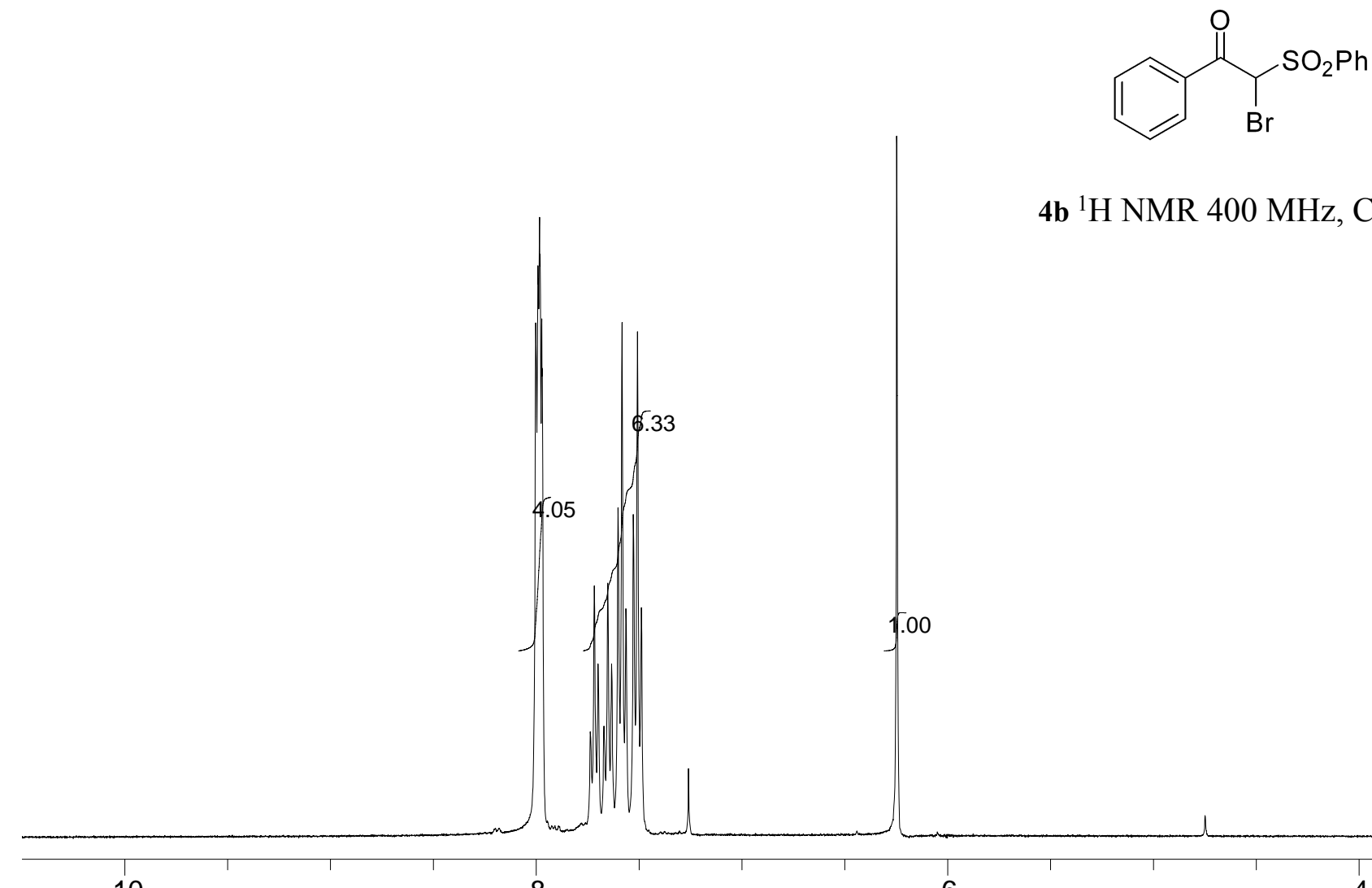

4b ${ }^{1} \mathrm{H}$ NMR $400 \mathrm{MHz}, \mathrm{CDCl}_{3}$ 

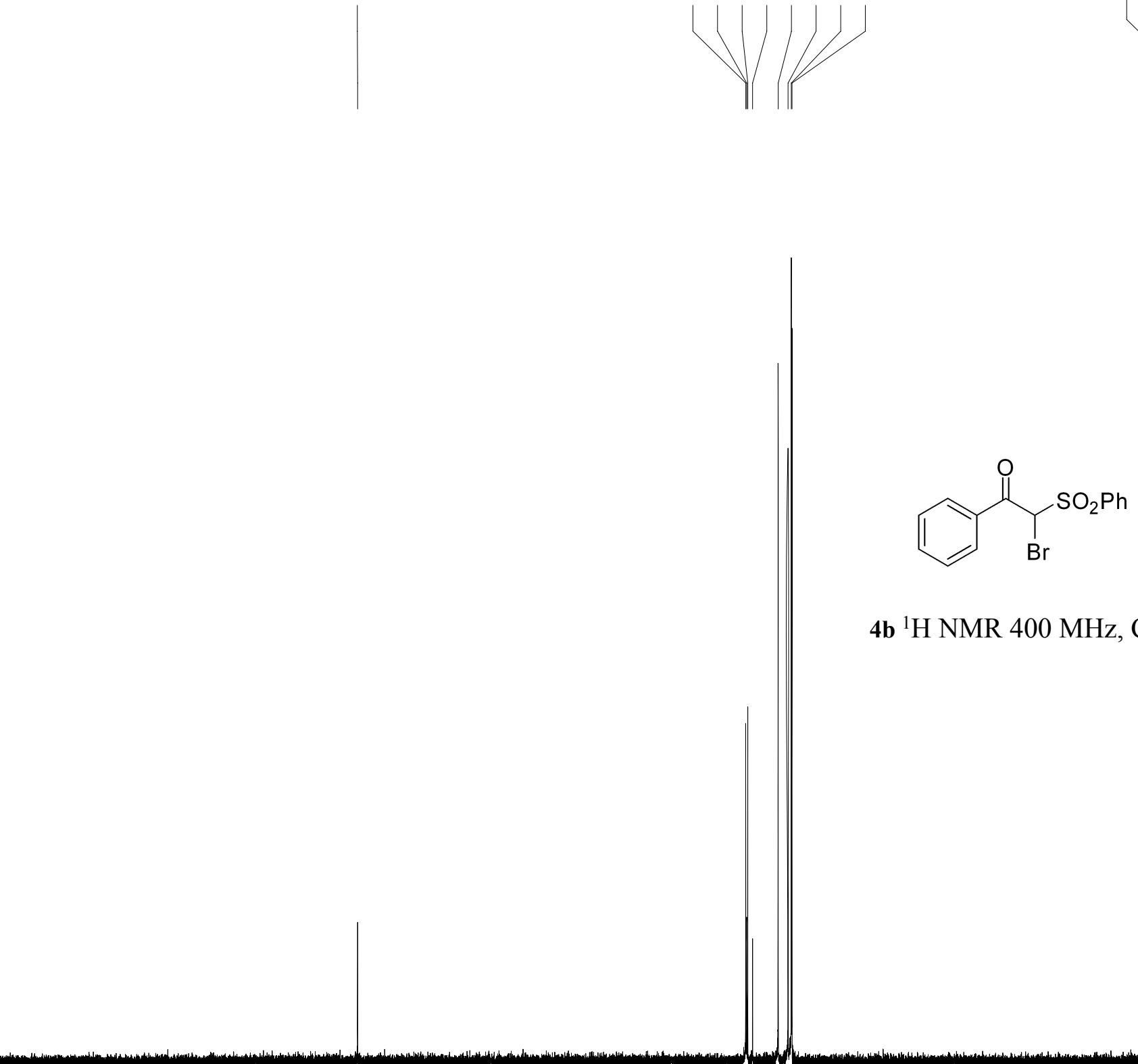

4b ${ }^{1} \mathrm{H}$ NMR $400 \mathrm{MHz}, \mathrm{CDCl}_{3}$ 

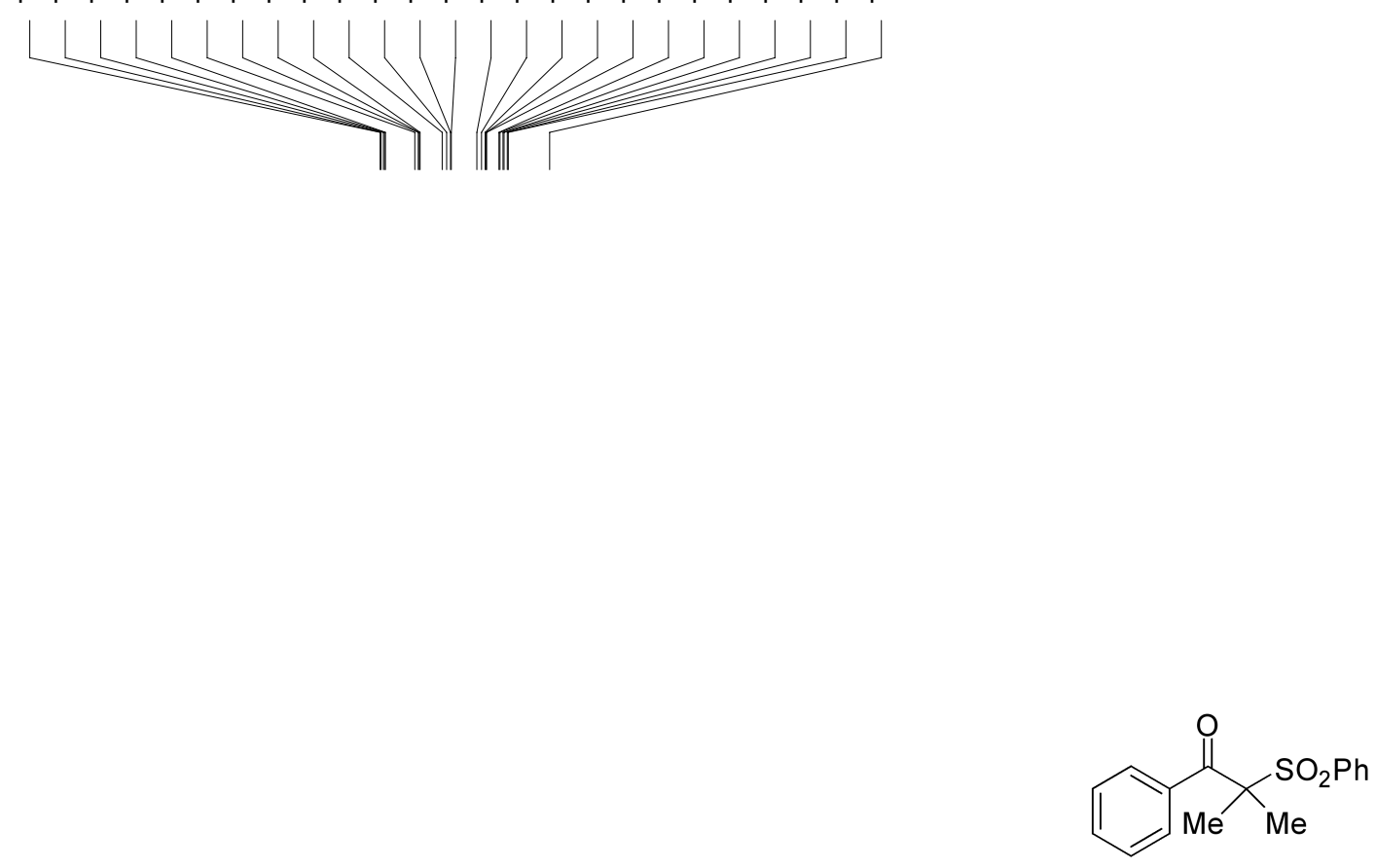

4c ${ }^{1} \mathrm{H}$ NMR $400 \mathrm{MHz}, \mathrm{CDCl}_{3}$

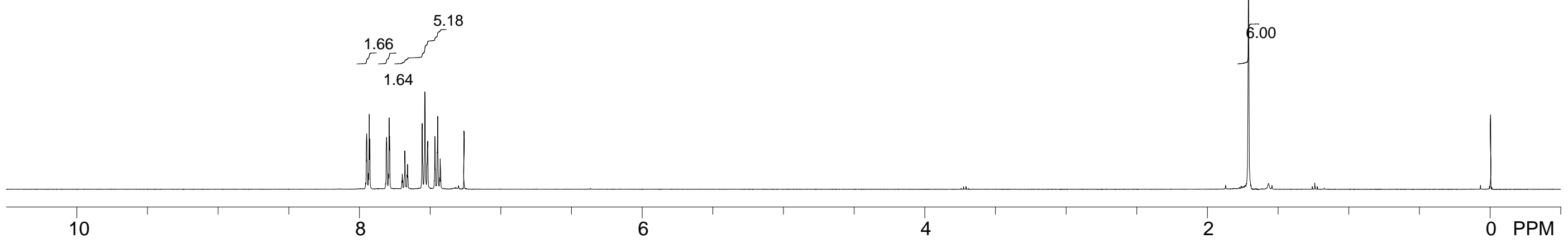




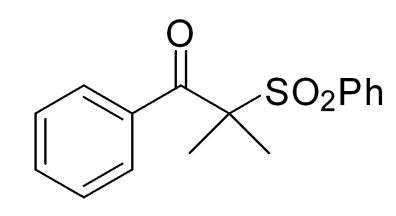

4c ${ }^{13} \mathrm{C}$ NMR $100 \mathrm{MHz}, \mathrm{CDCl}_{3}$ 


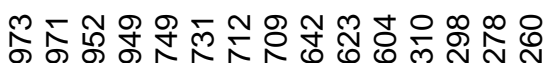

รำำ
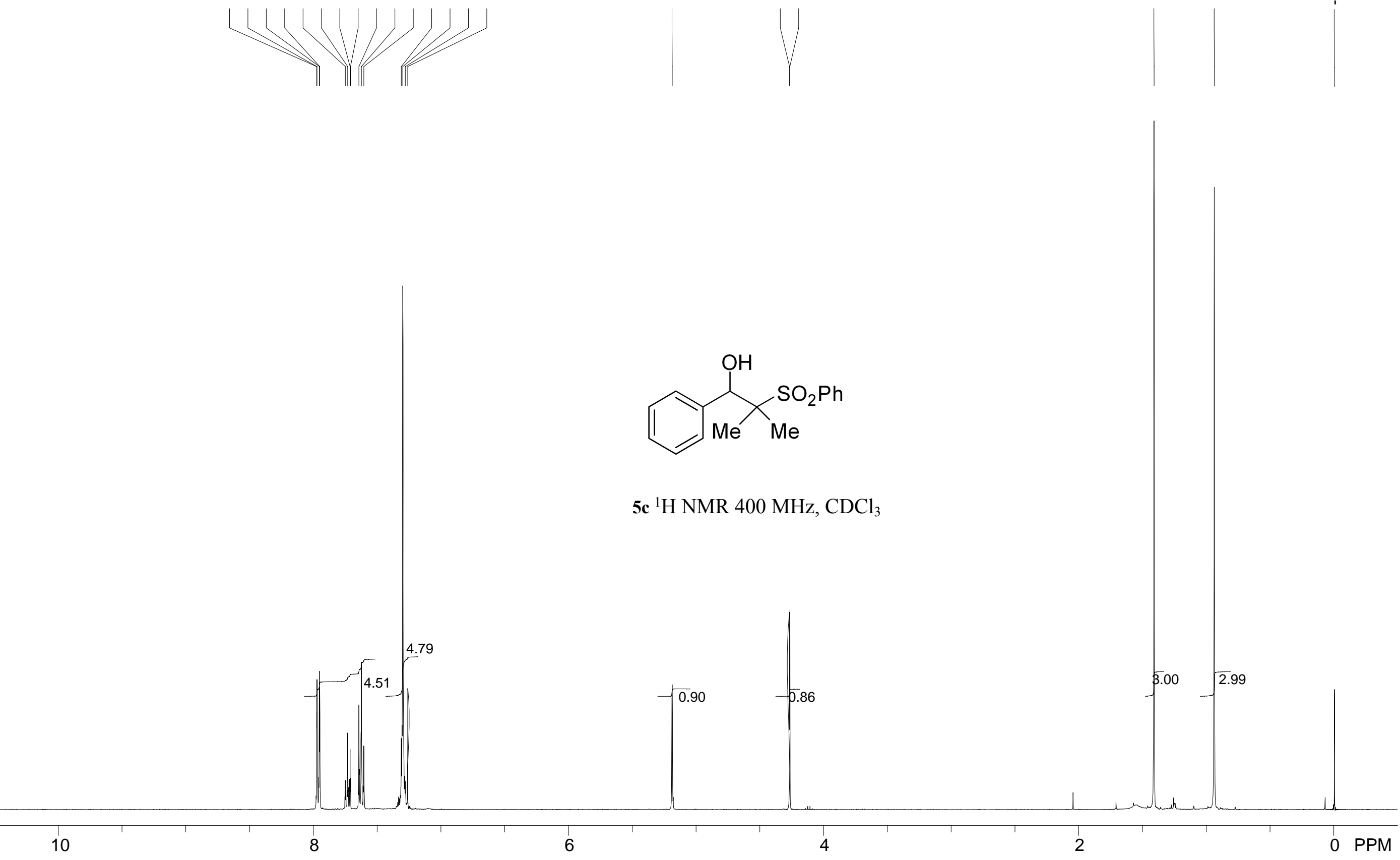


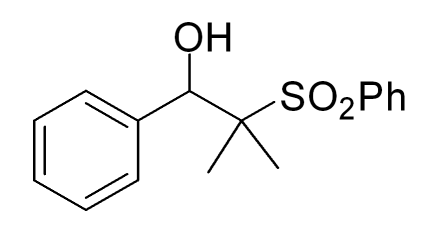

5c ${ }^{13} \mathrm{C}$ NMR $100 \mathrm{MHz}, \mathrm{CDCl}_{3}$ 


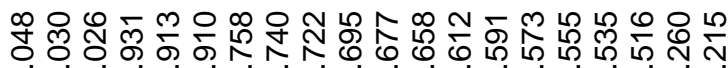

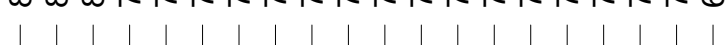
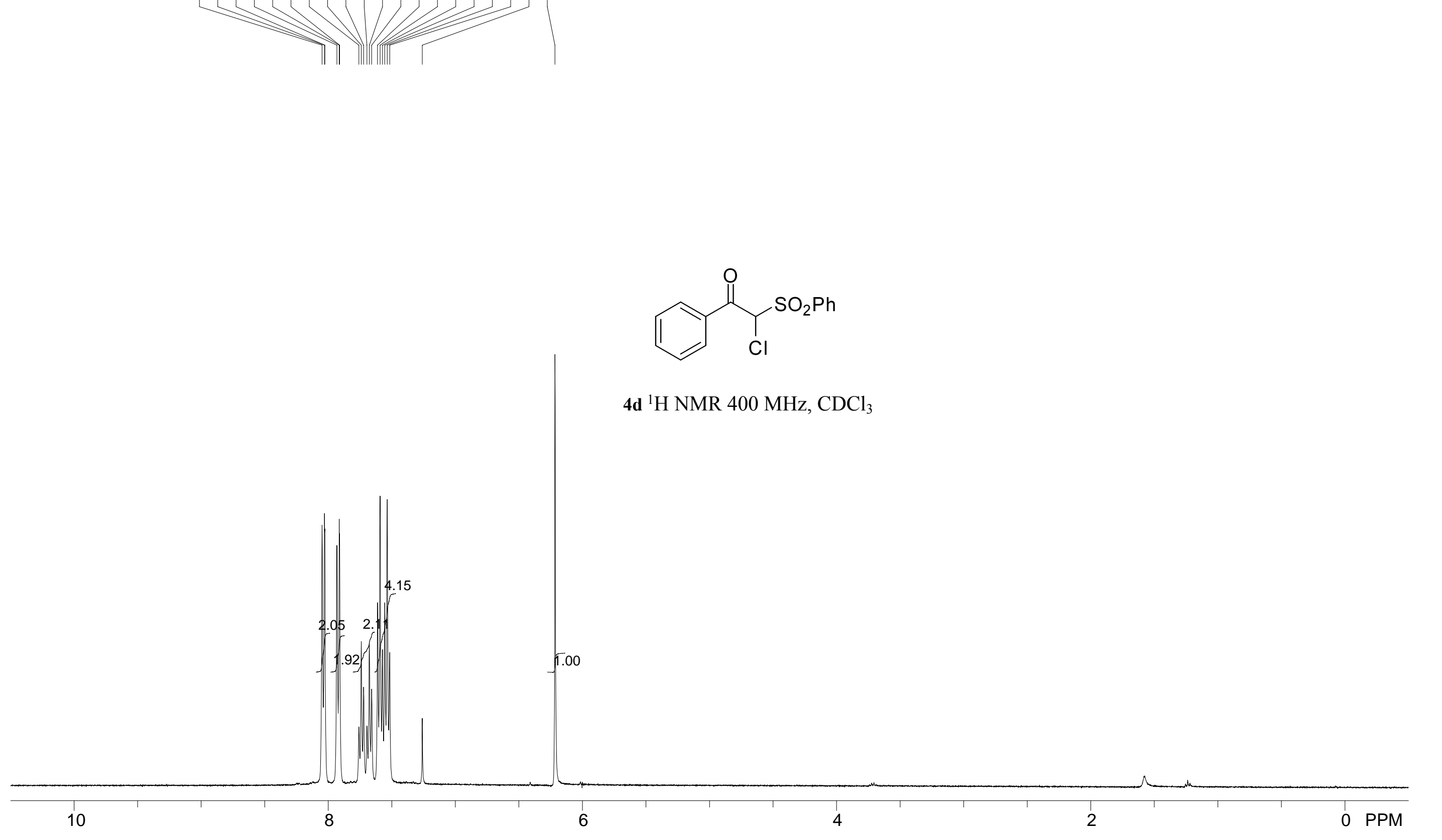


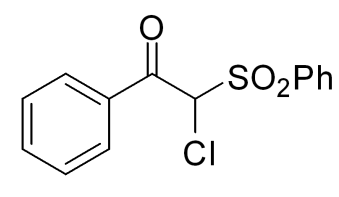

4d ${ }^{13} \mathrm{C}$ NMR $100 \mathrm{MHz}, \mathrm{CDCl}_{3}$ 

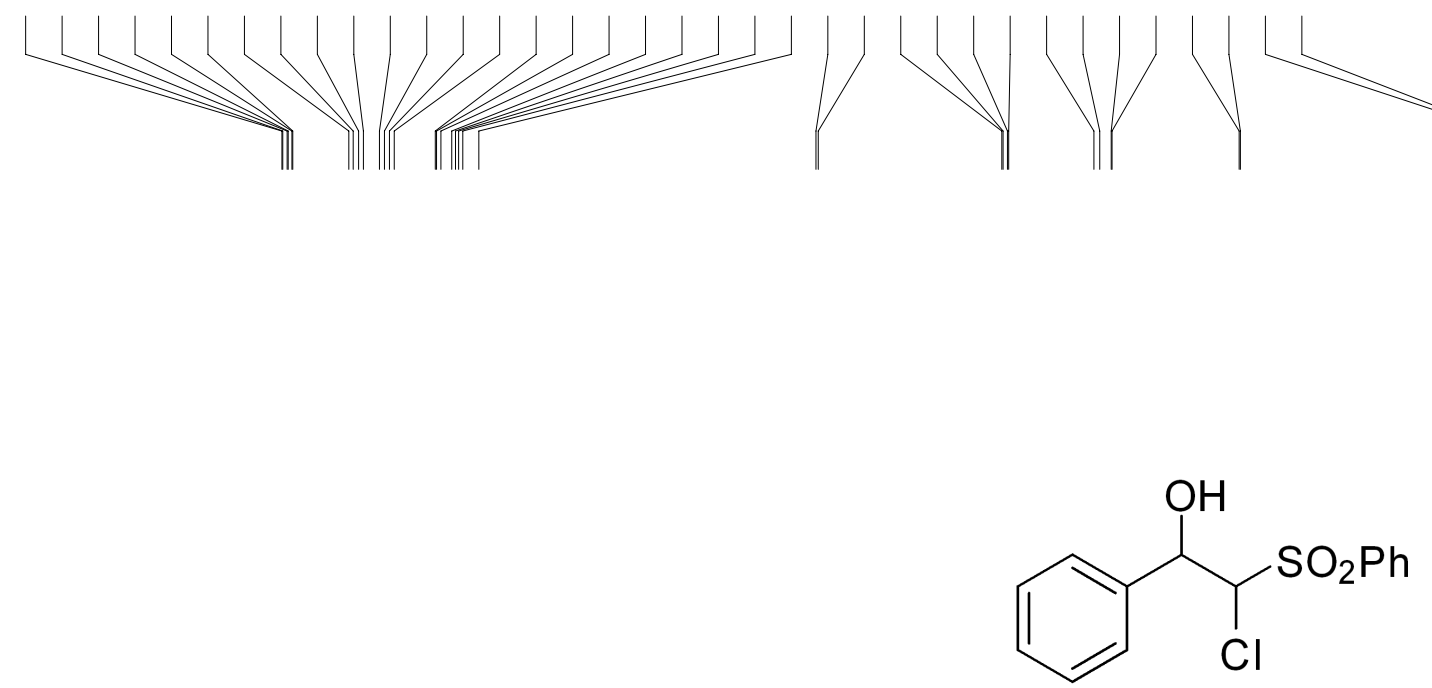

5d ${ }^{1} \mathrm{H}$ NMR $400 \mathrm{MHz}, \mathrm{CDCl}_{3}$ 


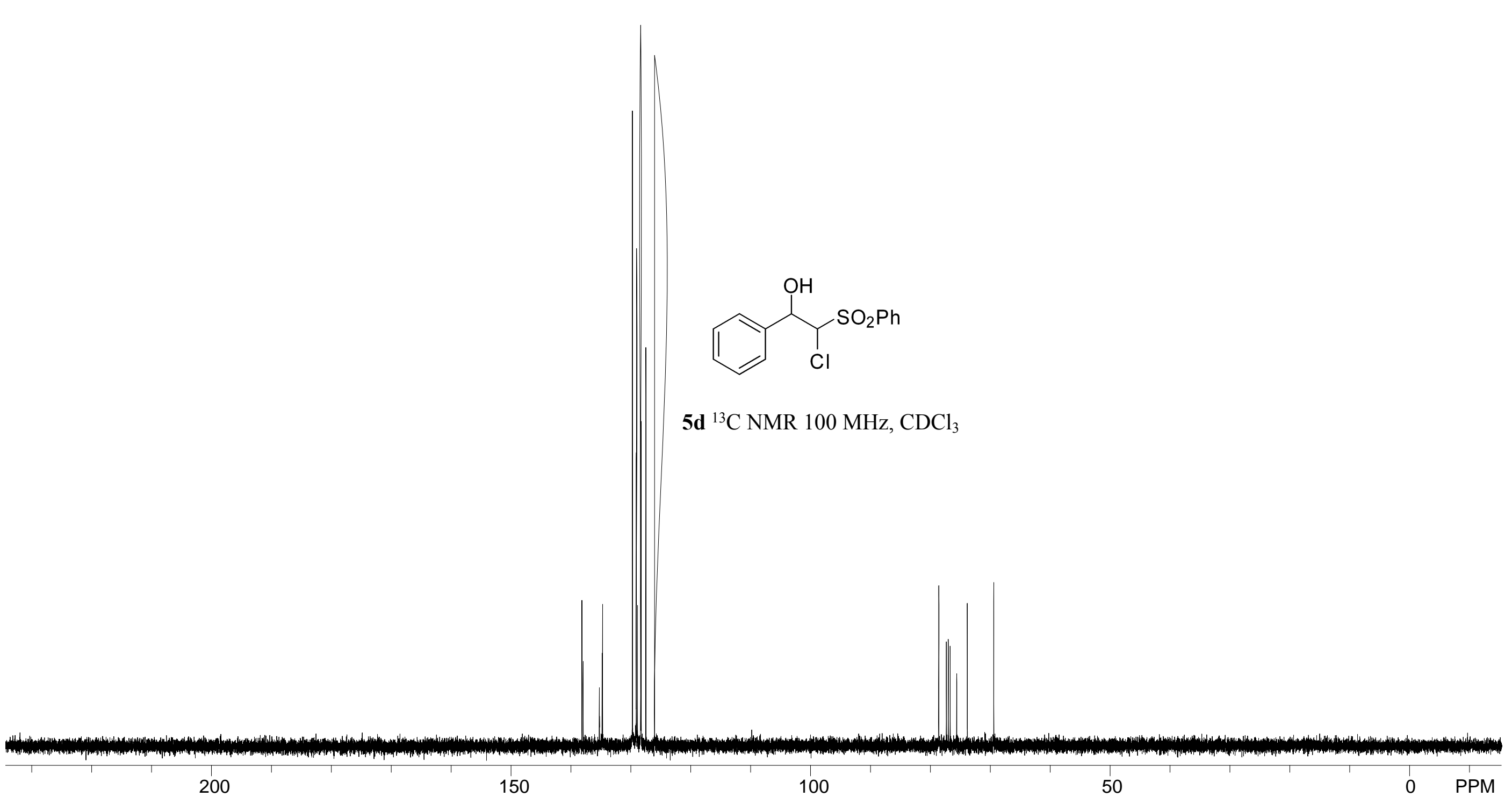

